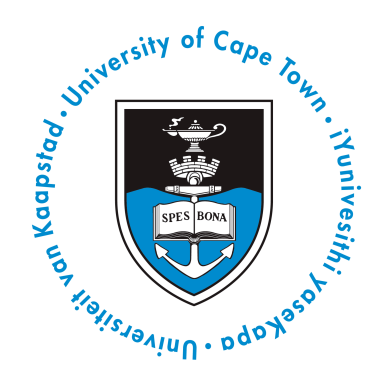

\title{
Enhancing Digital Heritage Archives Using Gamified Annotations
}

\author{
Mr. Job King'ori Maina
}

Supervisor:

A/Prof. Hussein Suleman

A dissertation presented for the degree of Master of Science in Computer Science

\author{
Department of Computer Science \\ Faculty of Science \\ University of Cape Town
}

Wednesday $15^{\text {th }}$ March, 2017 
The copyright of this thesis vests in the author. No quotation from it or information derived from it is to be published without full acknowledgement of the source. The thesis is to be used for private study or noncommercial research purposes only.

Published by the University of Cape Town (UCT) in terms of the non-exclusive license granted to UCT by the author. 



\section{License}

Job King'ori Maina is the author of this dissertation and holds copyright in terms of the University of Cape Town's Intellectual Property Policy.

This work is licensed by the author under a Creative Commons Attribution 2.5 South Africa (CC BY 2.5 ZA) License. 



\section{Plagiarism Declaration}

I, Job King'ori Maina, declare that I know the meaning of plagiarism and that all of the work in the dissertation, save for that which is properly acknowledged, is my own.

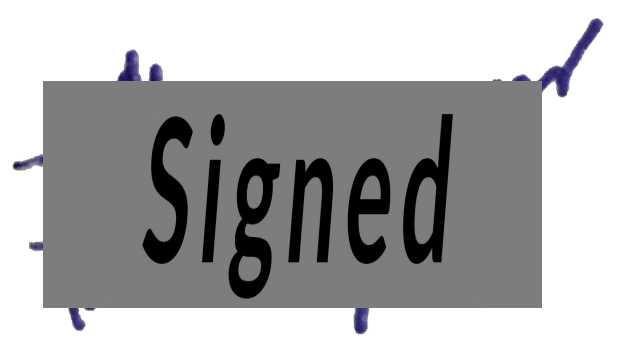

Wednesday $15^{\text {th }}$ March, 2017,

Job King'ori Maina,

E-mail: j@kingori.co,

Website: kingori.co. 



\section{Acknowledgments}

I would like to thank my supervisor, Associate Professor Hussein Suleman, who has been very patient with me throughout this period. I started the program with very little knowledge about how to follow through with a proper research process, nor having deep understanding as to how research plays a significant role in almost everything we interact with today. Several months later, I can attest to: a deep appreciation on the value of research in decision making, it's contribution to critical thinking, the hard work required to create a foolproof study, a deep respect for researchers and the importance of the preservation of heritage, to mention a few. Under his advisement, I have also picked up skills in LTEX, which is a plus in scientific writing.

My parents, Jackson Maina and Catherine Maina. They have been a continuous source of encouragement for me throughout this period. Their lessons and advice throughout the years have become particularly relevant to me this far away from home. My brother and sister, Jeff Maina and Esther Maina. Life in Cape Town would have been very difficult without their assistance.

I am also grateful to my good friend Shikoh Gitau. Through her, I was able to see first hand the impact that one's research can have globally. It is also through her gentle prodding that I decided to pursue an MSc. degree in Computer Science at UCT.

I would also like to acknowledge that this research was partially funded by the National Research Foundation of South Africa, University of Cape Town and Telkom SA Ltd. Any opinions, findings, conclusions or recommendations expressed in this publication are of the author, and the funders accept no liability whatsoever. 



\section{Abstract}

In the context of digital heritage archives, we find heritage objects having intrinsic contextual and historical information. Capturing all that information is difficult, especially if that effort is left only to the professionals or institutions responsible for those digital heritage archives.

In this study, we investigate how digital heritage archives can be enhanced using an annotation framework with a focus on gamification. So far, they have been focused on the collection of information and not really on the collaborative capabilities that they could have. We look at how we can add a collaborative element to an already existing digital heritage archive and incentivise users to engage with it more. This way, the owners present their data as the fixed content of the archive and the viewers are then able to present their contributions as annotations layered on the original work.

Therefore, using gamified annotations as a proposed solution, we hypothesise that gamification could play an important role in giving the participants an incentive as to why they should be engaging with the digital heritage archive as well as guiding them to contribute relevant content.

Through an experimental study, we found that gamified annotations do affect the number and quality of annotations submitted. We believe a successful implementation of a gamified annotation framework should go a long way to improve viewership, sharing, learning and debate around the content of the said digital heritage archives.

\section{Keywords}

gamification, digital archives, heritage archives, annotations, Bleek and Lloyd 



\section{Publications}

Some of the ideas and results derived from this research have been published in:

- J. K. Maina and H. Suleman. Enhancing digital heritage archives using gamified annotations. In B. R. Allen, J. Hunter, and L. M. Zeng, editors, Digital Libraries: Providing Quality Information. Proceedings of the 17th International Conference on Asia-Pacific Digital Libraries (ICADL), pages 169-179. Springer International Publishing, Seoul, Korea, 2015. ISBN 978-3-319-27974-9. doi: 10.1007/978-3-319-27974-9_17. URL http://dx.doi.org/10.1007/978-3319-27974-9_17 



\section{Table of Contents}

$\begin{array}{ll}\text { License } & \text { iii }\end{array}$

Plagiarism Declaration $\quad$ v

Acknowledgments vii

$\begin{array}{ll}\text { Abstract } & \text { ix }\end{array}$

$\begin{array}{lc}\text { Publications } & \text { xi }\end{array}$

$\begin{array}{ll}\text { List of Tables } & \text { xvii }\end{array}$

List of Figures $\quad$ xix

1 Introduction $\quad 21$

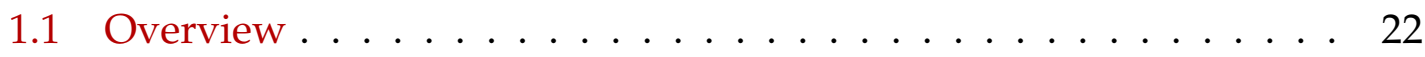

1.2 Problem Statement . . . . . . . . . . . . . . . . . . . . . . 22

1.3 Key Terminology . . . . . . . . . . . . . . . . . . 23

1.4 Motivating Case Study . . . . . . . . . . . . . . . . . . . 24

1.4.1 Introduction to Genius.com . . . . . . . . . . . . . 24

1.4 .2 How It Works . . . . . . . . . . . . . . . . . . . . . . 25

1.4 .3 Context, Adoption and Impact . . . . . . . . . . . . . . . 28

1.4.4 Why Is It Important? . . . . . . . . . . . . . . . . . . . . . . . . . . . . . 28

1.5 Context . . . . . . . . . . . . . . . . . . 28

1.6 Statement of Hypotheses . . . . . . . . . . . . . . . . . . . . . . . . . . . . . . . . . . . 39

1.7 Research Questions . . . . . . . . . . . . . . . . . . . . . . . . . 30

1.8 Research Approach . . . . . . . . . . . . . . . . . . . . . . . 31

1.9 Research Report Organisation . . . . . . . . . . . . . . 31

2 Literature Review 33

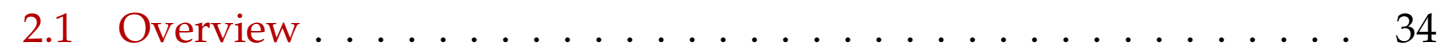

2.2 Annotations and Annotation Frameworks . . . . . . . . . . . . 34 
2.2.1 A Model for Annotations . . . . . . . . . . . . . . . . 34

2.2.2 Major Annotations Frameworks . . . . . . . . . . . 35

2.2.3 Comparison of Annotation Frameworks . . . . . . . . . . . . 38

2.2.4 Motivations for Annotation . . . . . . . . . . . . . . . 40

2.3 Annotations Within Digital Libraries . . . . . . . . . . . . . . . . . . 41

2.3.1 Examples of Digital Libraries With Annotations . . . . . . . 42

2.3.2 Results of Annotations on Digital Libraries . . . . . . . . . . 45

2.4 Gamification . . . . . . . . . . . . . . . . . . 47

2.4.1 Defining Gamification . . . . . . . . . . . . . . . 47 4

2.4.2 Does Gamification Work? . . . . . . . . . . . . . . . . . 47

2.4.3 Implementing Effective and Meaningful Gamification . . . . 48

2.4.4 Criticisms and Possible Pitfalls of Gamification . . . . . . . . 51

2.5 Gamification Within Digital Libraries . . . . . . . . . . . . . . . 52

2.6 Annotation Data Model Specifications . . . . . . . . . . . . . . . . . . . 53

2.6.1 Open Annotation Data Model . . . . . . . . . . . . . . . . . . 53

2.6.2 Web Annotation Data Model . . . . . . . . . . . . . . . . 55

2.7 Summary . . . . . . . . . . . . . . . . . . 57

3 System Design and Implementation $\quad 59$

3.1 Overview ............................ 60

3.2 Establishing Requirements . . . . . . . . . . . . . . . . . . . . . . 60

3.3 Confirming Requirements . . . . . . . . . . . . . . . . . 62

3.4 The Digital Bleek and Lloyd Collection . . . . . . . . . . . . . . . . . 64

3.4.1 The Source Data . . . . . . . . . . . . . . . . . . . . . 64

3.4.2 Recreating the Archive for Testing . . . . . . . . . . . . 65

3.5 System Overview . . . . . . . . . . . . . . . . . . . 66

3.5.1 Interaction With Text Annotations . . . . . . . . . . . . 67

3.5.2 Interaction With Image Annotations . . . . . . . . . . . . 71

3.5.3 Gamification Elements . . . . . . . . . . . . . . . 73

3.6 Building The Annotation Engine . . . . . . . . . . . . . . . . . 74

3.6.1 API Endpoints (Server-side) . . . . . . . . . . . . . 74

3.6.2 Annotator Library (Client-side) . . . . . . . . . . . . . . 80

3.7 Integrating Annotation Engine With The Digital Collection . . . . . 83

3.8 Technologies and Tools Used . . . . . . . . . . . . . . . . . . 85

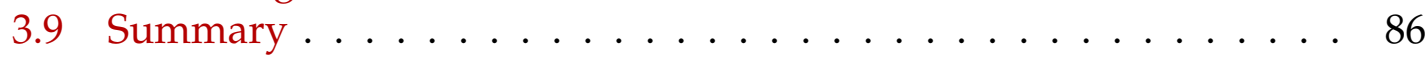

4 Experimental Design $\quad 89$

4.1 Overview ................................... 90

4.2 Usability Experiment . . . . . . . . . . . . . . . . . . 90

4.2.1 Usability Testing Prototype . . . . . . . . . . . . . . . . 990 90

4.2.2 Usability Evaluation Process . . . . . . . . . . . . . 91 
4.2.3 Addressing Feedback from Evaluation Process . . . . . . . . 94

4.3 Gamification Experiment . . . . . . . . . . . . . . . . . . . 94

4.3.1 Participation Process . . . . . . . . . . . . . . . . . 95

4.3 .2 Survey Questions . . . . . . . . . . . . . . . . . . . . . . . . 96

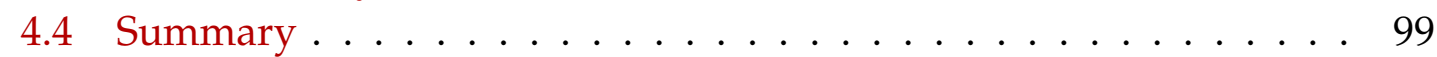

5 Results and Analysis $\quad 101$

5.1 Overview . . . . . . . . . . . . . . . . . . . 102

5.2 Research Questions . . . . . . . . . . . . . . . . . . . . . . 102

5.3 Usability Experiment Evaluation . . . . . . . . . . . . . . . . . . . . . . . . . . . 102

5.3.1 Experimental Data . . . . . . . . . . . . . . . . . 102

5.3.2 Usability Survey Analysis . . . . . . . . . . . . . . . . . . . . . . . . . 104

5.4 Gamification Experiment Evaluation . . . . . . . . . . . . . . . . . . . . . . . . . . . . . . . .

5.4 Experimental Data . . . . . . . . . . . . . . . . . . . . . . . . . . . . . . . . . . .

5.4 .2 Task Analysis . . . . . . . . . . . . . . . . . . . 111

5.4 .3 Survey Analysis . . . . . . . . . . . . . . . . . . . . . . . . . . . . . . . . . . . . . .

5.5 Summary . . . . . . . . . . . . . . . . . 117

6 Conclusion $\quad 119$

6.1 Overview . . . . . . . . . . . . . . . . . . . 120

6.2 Answers to Research Questions . . . . . . . . . . . . . . . . . . 120

6.2.1 Quantity of Annotations . . . . . . . . . . . . . . . . . 120

6.2.2 Quality of Annotations . . . . . . . . . . . . . . . 120

6.2.3 Appropriate for Digital Heritage Archives . . . . . . . . . . 121

6.3 Other Observations . . . . . . . . . . . . . . . . . . . . . 121

6.3 .1 User Habits . . . . . . . . . . . . . . . . . . . . . 121

6.3.2 Annotations as a Feedback Mechanism . . . . . . . . . . . . 121

6.3 .3 Ease of Implementation . . . . . . . . . . . . . . . . 122

6.4 Contributions . . . . . . . . . . . . . . . . . 123

6.5 Future Work . . . . . . . . . . . . . . . . . . . . . 123

6.5.1 Display of Multiple Annotations . . . . . . . . . . . . . . . . . . . . 123

6.5 .2 Moderation of Annotations . . . . . . . . . . . . . . . . . . . 124

6.5.3 Supporting Discourse in Annotations . . . . . . . . . . . . 124

6.5.4 Annotating Other Types of Content . . . . . . . . . . . . . 124

$\begin{array}{ll}\text { Bibliography } & 127\end{array}$

$\begin{array}{lr}\text { A Annotator Store Rails Engine } & 139\end{array}$ 
B User Feedback on the Enhanced Digital Heritage Archive 141

B.1 Positive Aspects . . . . . . . . . . . . . . . . . . . . . . . . . . 141

B.2 Negative Aspects . . . . . . . . . . . . . . . . . . . . . 142 


\section{List of Tables}

1.1 Research approach process summary . . . . . . . . . . . . . . 31

2.1 Default Annotator JS plugins _. . . . . . . . . . . . . . . . . . 39

2.2 Taxonomy of tagging motivations from a study using ZoneTag/Flickr (a photo tagging application) [28]. . . . . . . . . . . . . . . 41

2.3 Theoretical base of incentives and rewards by Richter, Raban, and Rafaeli [77]. . . . . . . . . . . . . . . . . . . . . . . . 49

3.1 Comparison of features and characteristics of four major modern annotation platforms. . . . . . . . . . . . . . . . 61

3.2 Checklist of the fulfilment of functional requirements vis-a-vis selected annotation systems. . . . . . . . . . . . . . . . . . . 63

3.3 Checklist of the fulfilment of data requirements vis-a-vis selected annotation systems. . . . . . . . . . . . . . . . . . . . . 63

3.4 Checklist of the fulfilment of technical requirements vis-a-vis selected annotation systems. . . . . . . . . . . . . . . . . . 63

3.5 Annotator Store API endpoints where :id should be replaced with the annotation's actual id. . . . . . . . . . . . . . . . . 75

3.6 Image Annotation API endpoints . . . . . . . . . . . . . 82

4.1 Survey questions to measure the usefulness of the usability testing prototype. . . . . . . . . . . . . . 92

4.2 Survey questions to measure how easy it is to use the usability testing prototype. . . . . . . . . . . . . . . . 92

4.3 Survey questions to measure how easy it is to learn how to use the usability testing prototype. . . . . . . . . . . . . . . . 93

4.4 Survey questions to measure the users' satisfaction while using the usability testing prototype. . . . . . . . . . . . . 93

4.5 Survey questions to evaluate user motivations for viewing, contributing, revisiting and sharing the archive. . . . . . . . . 98 
4.6 Survey questions to evaluate usability of the core functions of the system. . . . . . . . . . . . . . . . . . 98 98

5.1 Usability study participants' content consumption habits. . . . . . . 103

5.2 Usability study participants' content generation habits. . . . . . . . . 103

5.3 Survey participants' previous history with digital archives. . . . . . 110

5.4 Ranked list of the 25 users who annotated in order of their total points scored calculated from images/text annotations and upvotes. The user ranked $13^{\text {th }}$ splits the results into two halves. . . . . 111

5.5 Breakdown of 387 annotations by relevance of annotation content. . 112 


\section{List of Figures}

1.1 Annotation on Genius.com of the phrase "hang him in a bottle like a cat and shoot him" found in the first act of Shakespeare's "Much Ado About Nothing". . . . . . . . . . . . . . . . . . . 25

1.2 List of all-time contributors on Genius.com in order of 'IQ points'. . 26

1.3 List of the 10 top-ranking contributors in the rap category on Genius based on 'IQ points'. It's possible to view ranking within different periods, that is: all-time, monthly or weekly. . . . . . . . . . . 27

2.1 Illustration of the basic annotation data model by Sanderson, Ciccarese, and Van de Sompel [82]. . . . . . . . . . . . . . . . . . . . 54

3.1 The Digital Bleek \& Lloyd Collection. . . . . . . . . . . . . . . . . . 65

3.2 Component digram illustrating how every element of the system ties in together. . . . . . . . . . . . . . . . . . 68

3.3 Blue pen tooltip widget (top right) used to aid in discovery of text annotation feature. . . . . . . . . . . . . . . . . 69

3.4 Annotation widget used when creating and editing a text annotation (with permission options below the text box). . . . . . . . 69

3.5 Annotation widget used when viewing an annotation (edit and delete buttons are on the top right). . . . . . . . . . . . . 70

3.6 Annotation view widget showing threaded annotations (multiple annotations on same portion of text). . . . . . . . . . . 70

3.7 White feather widget (top left of photo) used to aid in discovery of the image annotation feature. . . . . . . . . . . . . . 71

3.8 Annotation widget used when creating and editing an image annotation (with permission options below the text box). . . . . . . 72

3.9 Annotation widget used when viewing an image annotation (edit and delete buttons are on the top right). . . . . . . . . . . . 72

3.10 Leaderboard showing the usernames of different users and their respective scores which were derived from totals of annotations and up-votes. 6

\section{7}

\section{4} 65 68 69 9 
5.1 Graphed survey responses giving a measure of the usefulness of the usability testing prototype. . . . . . . . . . . . . . . 104

5.2 Graphed survey responses giving a measure of how easy it is to use the usability testing prototype. . . . . . . . . . . . . 105

5.3 Graphed survey responses giving a measure of how easy it is to learn how to use the usability testing prototype. . . . . . . . . 106

5.4 Graphed survey responses giving a measure of the users' satisfaction while using the usability testing prototype. . . . . . . . 106

5.5 Distribution of 112 users by their annotation activity. . . . . . . . . 110

5.6 Distribution of 387 submitted user annotations by type. . . . . . . . 110

5.7 User feedback on features that promote or motivate viewing more of the digital heritage archive. . . . . . . . . . . . . . . . 113

5.8 User feedback on features that promote or motivate contributing to the digital heritage archive. . . . . . . . . . . . . . . . . . . . . . . . 114

5.9 User feedback on features that promote or motivate revisiting of the digital heritage archive. . . . . . . . . . . . . . . . . . . . . 114

5.10 User feedback on features that promote or motivate sharing the digital heritage archive with others. . . . . . . . . . . . . . . . . . . 115

5.11 Survey responses giving a measure of the ease of use of the system according to gamified users. . . . . . . . . . . . . . 116

5.12 Survey responses giving a measure of the ease of use of the system according to un-gamified users. . . . . . . . . . . . 116 


\section{Chapter 1}

\section{Introduction}

\section{Contents}

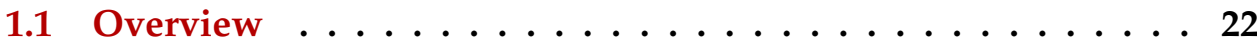

1.2 Problem Statement . . . . . . . . . . . . . . 22

1.3 Key Terminology . . . . . . . . . . . . . . . . 23

1.4 Motivating Case Study $\ldots \ldots \ldots \ldots \ldots$

1.4 .1 Introduction to Genius.com . . . . . . . . . . . . 24

1.4 .2 How It Works . . . . . . . . . . . . . . . . . 25

1.4.3 Context, Adoption and Impact . . . . . . . . . . . 28

1.4 .4 Why Is It Important? . . . . . . . . . . . . . . 28

1.5 Context ....................... 28

1.6 Statement of Hypotheses . . . . . . . . . . . . . 29

1.7 Research Questions . . . . . . . . . . . . . . . 30

1.8 Research Approach . . . . . . . . . . . . . . . 31

1.9 Research Report Organisation . . . . . . . . . . . . 31 


\subsection{Overview}

This chapter serves as an introduction to the research topic and provides a definition of its scope.

\subsection{Problem Statement}

The main reason that we have digital collections online is so that we can invite students, researchers, teachers, and the public to explore and connect with our past. Historians, librarians, archivists, and curators who share digital collections and exhibits measure their success in moving toward this goal by how people use, reuse, explore and understand these objects [76].

Following from that, digital heritage archives so far have primarily focused on the collection of information and not really on the discussion that occurs around that information. Nor do they focus on features that allow the viewers to participate. As a result, they have largely become collections of work by professionals in the domain that the digital heritage archive is targeted towards.

While widespread digitisation of heritage data is certainly a step in the right direction, it can pose an interesting problem going forward. These resulting digital heritage archives have become highly specialised environments, thus making it more difficult to instigate and enhance engagement with the archives by the viewers [49].

And so, to better understand the needs of the viewers of digital collections, it is important to break them down into different communities of interest. These are [91]:

- Professional researchers: These are established academics, experienced in the general area covered by the resource, but not necessarily connected with the specific content of the resource.

- Apprentice investigators: These are students at advanced undergraduate and postgraduate level. They have some knowledge of the historical period and/or cultural context addressed by the resource.

- Informed users: These are researchers who are not professional academics but have knowledge of some aspects addressed by the resource.

- General public: These are adults and children interested in the subject of the digital heritage collection.

While each of the four groups above has different needs when it comes to digital collections, there is still a considerable overlap. The study by Sweetnam 
et al. reveals some of these basic user requirements that were found to be common across each of the user groups that they surveyed, such as the ability to [91]:

- Perform accurate searches.

- Bookmark the results of a search to retrieve or recreate a given search filter easily.

- Interact with the content in a visual way such as by using maps.

- Add in-line annotations to items in the digital collection by highlighting portions of text and images.

- Have a log of interactions with the digital collection, which could power a recommendations or suggestions feature.

In addition to these, each user group had specific requirements that ultimately aimed to personalise the collection, enrich it or enhance their developing engagement with its contents. The professional researchers had the most specific and advanced requirements, followed by the apprentice investigators, informed users and finally the general public [91]. The transfer of knowledge was such that the general users would rely on the information shared by the more experienced and knowledgable user groups.

The apprentice investigators hoped to benefit from the exposed work carried out by the more experienced professional researchers. The informed users in turn had less intensive requirements than the apprentice investigators but at the same time had more detailed requirements than the general public. Lastly, the general public had very little contextual information about the collection and identified the need for accessible introductions to the collections, which would explain the material they contain and its historical context [91].

It is on that final note on the general public that this research aims to explore and focus. We seek to find out how to encourage the participation of all groups in digital archives using annotations in a way that promotes transference of information from the more knowledgable groups to the less knowledgable groups.

\subsection{Key Terminology}

Below are the three main terms used repeatedly in this study:

Annotation This is a critical or exploratory note or body of notes added to a text. ${ }^{1}$ The Merriam-Webster dictionary expands this definition from text, stating

\footnotetext{
${ }^{1}$ http://dictionary.reference.com/browse/annotation
} 
that an annotation is a note that is added to text, book, drawing, etc., as a comment of explanation. ${ }^{2}$ This is to show that annotations can be applied to other forms of media as well.

However, in the context of this research, annotations refer to those notes made on specific portions of the document to which they are attached and not the entire document. The types of media that we will apply annotations to will also be limited to images and text.

Digital Heritage Archive This may also be referred to as a digital heritage library, digital heritage repository, digital heritage collection or even digital cultural heritage library. It is a special library that is focused on the collection of digital objects that may include text, image, audio, video and other types of digital media that are related to or provide contextual information on heritage and culture. Heritage and culture may be used interchangeably but, for the sake of consistency, heritage will be the primary term in use throughout.

Gamification This is largely defined as the use of game design elements in nongaming contexts to motivate users, increase user-activity and user-retention. It is often implemented as a service layer of reward and reputation systems such as points, badges, levels and leader boards. Alternatively, Deterding et al. note that some vendors and consultants tend to describe it in terms of client benefits, for example: as the process of integrating game dynamics into a site, service, community or content of a campaign, in order to drive participation [41].

\subsection{Motivating Case Study}

\subsubsection{Introduction to Genius.com}

Genius.com, formerly and popularly known Rap Genius, was at its conception a rap lyrics only website but can now be regarded as an online knowledge base $e^{3}$. The site allows its users to add context and interpretations to text and images through an annotation system.

Starting off as a website with no intentions of expanding beyond rap lyrics, the website has slowly grown to include other genres such as literature ${ }^{4}$, speeches $^{5}$,

\footnotetext{
${ }^{2}$ http://www.merriam-webster.com/dictionary/annotation

${ }^{3}$ A store of information or data that is available to draw on.

${ }^{4}$ The Seven Ages of Man by William Shakespeare. http://lit.genius.com/William-shakespeare-the-seven-ages-of-man-annotated

${ }^{5}$ The Gettysburg Address by Abraham Lincoln. http://history.genius.com/Abraham-lincoln-the-gettysburg-address-annotated
} 
TV and movie scripts ${ }^{6}$, restaurant menus ${ }^{7}$, drug warnings ${ }^{8}$ and even sports rosters $^{9}[64]$.

\subsubsection{How It Works}

Each annotation layers extra information on top of the content, enabling the reader to understand its context as they read. These annotations can be composed of varying media types such as text, still images, motion images like GIFs ${ }^{10}$ and even embedded videos from external online services such as YouTube. See Figure 1.1 .

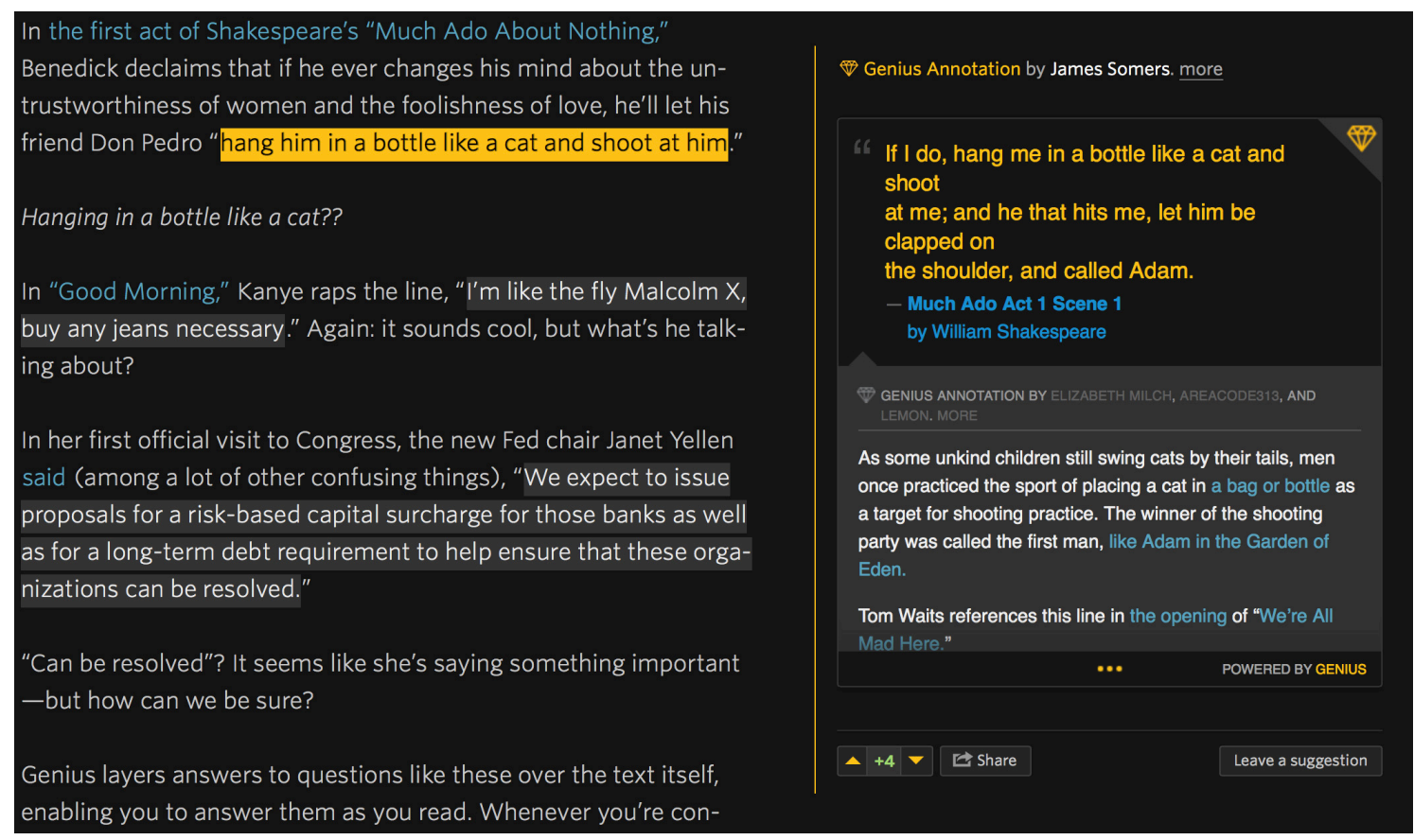

Figure 1.1: Annotation on Genius.com of the phrase "hang him in a bottle like a cat and shoot him" found in the first act of Shakespeare's "Much Ado About Nothing".

\footnotetext{
${ }^{6}$ Ozymandias episode from Breaking Bad.

http://screen.genius. com/Breaking-bad-ozymandias-annotated

${ }^{7}$ Chipotle Mexican Grill, Inc. menu.

http://x.genius .com/Chipotle-mexican-grill-inc-menu-annotated

${ }^{8}$ TYLENOL ${ }^{\circledR}$ Regular Strength Tablets warning.

http://x.genius . com/Tylenol-regular-strength-tablets-warnings-annotated

${ }^{9}$ The roster of the 1986 New York Mets.

http://sports.genius. com/Mlb-rosters-1986-new-york-mets-annotated

${ }^{10}$ Graphics Interchange Format
} 
An annotation therefore is like a miniature Wikipedia page with constantly improving distillations of the combined wisdom of scholars. As a result, Genius becomes a conversation built around texts and the interpretations of those texts [8].

Users of Genius are incentivised to participate through a system of reputation and reward. Each user can earn reputation in the form of 'IQ points' for various actions and reactions on the site, for example: writing an annotation; getting your annotation up-voted; or moderating someone else's work [8].

The reference to these points as IQ points seems to have the intention of giving the annotation effort a scholarly connotation and users with the highest IQ points automatically get recognised as scholars in a particular category or body of work.

This recognition is acknowledged via leaderboards that rank the users by the IQ points that they have been able to accrue. See Figure 1.2 and Figure 1.3. In the former, the leaderboard lists users ranked on the total IQ points awarded across all categories and, in the latter, we see a similar leaderboard scoped to the Rap category.

\section{The Genius Contributors}

\section{SameOldShawn}

373,321

Magnitude901

180,121

Nightwing

146,713

Nebja

115,612

IllustriousQ

110,458

Clément RGF

107,794
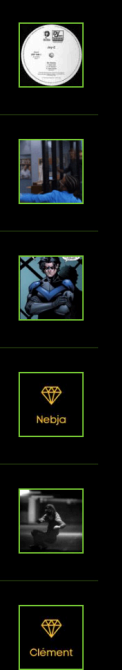

Maboo

349,675

DC26

167,113

Guillaume Simonin

133,239

Palacelight

114,659

Tyrant

107,986

ScopeY

104,156

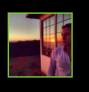

streetlights

208,909

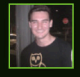

Heisenferg

150,485

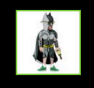

Barrk

127,184

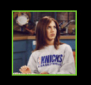

EwokABdevito

113,759

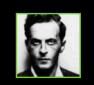

MrXercules

107,816

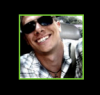

Romi Ezzo

99,861

Figure 1.2: List of all-time contributors on Genius.com in order of 'IQ points'.

IQ points are also directly tied to access levels on the site, namely: editors, moderators, regulators and users who have to acquire IQ points to access these privileges.

An editor is a contributor who has proven that they can consistently write high-quality annotations [9]. 


\section{Rap IQ Leaderboard}

\section{All-time}

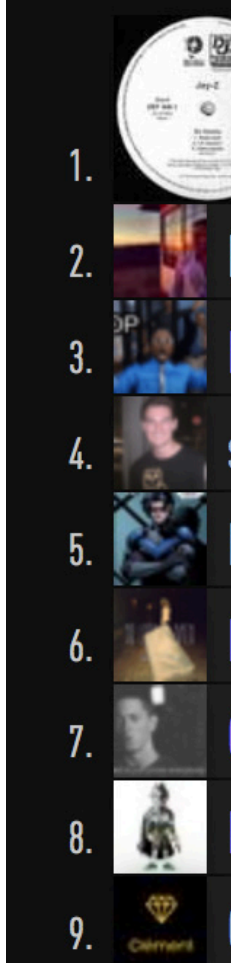

SameOldShawn

\section{Weekly}

Monthly

Maboo

285,577

Magnitude901

176,520

streetlights

169,468

Nightwing

142,440

DC26

138,345

Guillaume Simonin

111,812

Heisenferg

110,930

Clément RGF

103,192

10. Nebja

Figure 1.3: List of the 10 top-ranking contributors in the rap category on Genius based on 'IQ points'. It's possible to view ranking within different periods, that is: all-time, monthly or weekly.

Editors, for instance, can accept or reject suggestions from other users and even edit and delete other people's annotations [9]. Moderators have all the access that editors have but with the extra ability of being able to make other contributors into editors, delete and even lock/unlock content [10]. Regulators, on the other hand, are largely responsible for the health of the Genius community and have all the access that editors and moderators have but with the extra ability of being able to make others into moderators [11].

Annotations on Genius are crowd-sourced from the community and this adds authenticity to the annotations. Genius also allows 'verified annotations'. The only difference between verified and normal annotations is that they are made by per- 
sons responsible for or closely related to the referent. This could be the artist, producer or creator of the document [8].

\subsubsection{Context, Adoption and Impact}

Rap's perception, of being viewed to be trivial or indecipherable and too ethnic, made it the perfect candidate for annotation. The reason for this was because one needed to know the circumstances that form the setting for the event, statement, or idea covered by the referent to fully understand the content [54].

Horowitz argues that we need knowledge of the culture, history, and people to fully understand the references in rap [54]. Annotations, in this specific realworld application then became the suitable technology layer that enabled 'scholars' to crowd-source the most correct interpretation of or meaning in a certain section of text, image or video.

\subsubsection{Why Is It Important?}

From this case study, we get some key ideas that define the logic, direction and intended goal of our research study. These ideas are that: i) annotations are a way to add layers of meaning to archives; and $i i)$ users often have to be incentivised to add annotations to an archive .

This feeds into the idea that annotations could be that solution that could increase engagement and participation as well as provide context and meaning to the digital heritage objects, especially from a user's perspective if other users are incentivised to annotate.

\subsection{Context}

Over the past few decades, a lot of work has gone into how annotations are implemented on the Web. Previous research in the Web annotations domain has focused on creating open standards to define annotations $[51,56,86]$. These standards have evolved from supporting simple text annotations [56] to other multimedia types like: images, audio and video [86]. Other research has focused on aspects such as creating timeless annotations [81].

In the context of digital heritage archives, annotations could be the middle ground, where experts collate information in digital repositories and users participate in a layer of information above the fixed expert's contributions. This layer could hold the key to rich metadata/contextual information, a Semantic Web and successful contribution system. 
Collective participation in digital archives is not a new concept and has been explored, researched and even implemented by many. A good example would be Wikipedia, which is a digital archive that is publicly editable by anyone. Yet, despite its success, Wikipedia does have its fair share of flaws and critics have not wasted time to point them out $[29,63]$.

Gamification, as applied in Genius (see Chapter 1.4), could be the catalyst that gets participants engaged, as they are essential to the success of any collaborative environment. Without them, even the most well designed and structured annotation system with a good user-interface would not live up to its purpose.

If gamified annotations are implemented well, they could play an important role in giving the participants an incentive as to why they should be engaging with the digital heritage archive. This would be particularly helpful in the context of heritage archives, where we find heritage objects having intrinsic contextual and historical information. Such information is hard to capture, especially if that effort is left alone to the individuals or institutions responsible for those heritage archives.

With a successful annotation effort, the responsibility of maintaining the fixed data is left untouched while the contextual information is outsourced to the participants.

The case for digital heritage archives is that this could go a long way to provide rich metadata for younger generations to make use of and learn more about heritage objects. After all, previous research confirms that digital archive resources facilitate better learning when compared to search engine sources [36]; it is therefore important that we look into features that could enhance that engagement experience through participation and discourse.

It is on this idea of gamified annotations that we build our research. Our goal is to investigate if gamifying the annotation process would lead to more engagement with annotations on a digital heritage archive.

\subsection{Statement of Hypotheses}

This research is based on the working hypothesis that gamified annotations can improve the number and quality of annotations submitted to a digital heritage archive, thus improving engagement, collaboration and learning benefits for the user. 


\subsection{Research Questions}

In this study our goal will be to satisfactorily answer the following research question:

Are gamified annotations an effective tool to increase the number and quality of annotations submitted to a digital heritage archive?

The above research question broadly seeks to investigate the applicability of gamification in the specific context of a digital heritage archive. Two aspects of this applicability are elaborated below:

a) Are gamified annotations an appropriate approach for digital heritage archives?

Annotation can add value to heritage data. However, annotation is known to work better with some types of content than others, i.e. it is easier to annotate images and text than it is to annotate video.

It is also a broad assumption that gamification would automatically trigger interest in submitting to a digital heritage archive regardless of content.

b) Does the gamification of annotations improve the number and the quality of annotations submitted in digital heritage archives?

The goal was to determine if gamifying annotations would motivate users to annotate digital objects and observe if there are any other secondary results such as improved learning and engagement. 


\subsection{Research Approach}

A summary of the process followed to carry out this research is presented in Table 1.1 .

\begin{tabular}{l|l} 
Research Process & Procedure \\
\hline $\begin{array}{l}\text { Literature synthesis } \\
\text { Research proposal }\end{array}$ & $\begin{array}{l}\text { Preliminary review of existing literature. } \\
\text { Scoping and formulation of the research } \\
\text { problem. } \\
\text { Building an experimental prototype us- } \\
\text { ing an already existing digital heritage } \\
\text { archive. } \\
\text { Testing the usability of the built system } \\
\text { with actual users. } \\
\text { Carry out final experiment to answer the } \\
\text { Usability Evaluation } \\
\text { Final Evaluation }\end{array}$ \\
Analysis & $\begin{array}{l}\text { Analyse experimentation results and dis- } \\
\text { cussion. }\end{array}$
\end{tabular}

Table 1.1: Research approach process summary

\subsection{Research Report Organisation}

This thesis is organised into 6 chapters, briefly described below:

- Chapter 1 is an introduction to the research. It goes through the problem being tackled, the motivating case study, research questions and clearly defines the scope of this research.

- Chapter 2 gives a comprehensive review of previous work related to this research. It describes the fields (annotation and gamification) in general and highlights how others have tried to solve these problems and their observations. Its purpose is to give the right context within which we can validate the experiments, evaluation, results, recommendations and conclusions of this research.

- Chapter 3 discusses the design and implementation of the systems developed for purposes of this resaerch. 
- Chapter 4 describes the methods used to carry out this research. The core method will be centred on an experimental prototype.

- Chapter 5 presents the results of the research experiment and their analysis. This also includes details of the evaluation process.

- Chapter 6 includes concluding statements, approaches that seem promising to overcome problems encountered (but were not tackled) and also suggestions for potential future work that may fill any gaps in this research study. 


\section{Chapter 2}

\section{Literature Review}

\section{Contents}

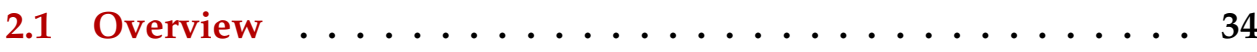

2.2 Annotations and Annotation Frameworks . . . . . . . . . 34

2.2.1 A Model for Annotations . . . . . . . . . . . . . . . . . . . 34

2.2.2 Major Annotations Frameworks . . . . . . . . . . . 35

2.2.3 Comparison of Annotation Frameworks . . . . . . . . . . 38

2.2.4 Motivations for Annotation . . . . . . . . . . . . . . . . . 40

2.3 Annotations Within Digital Libraries . . . . . . . . . . . 41

2.3.1 Examples of Digital Libraries With Annotations . . . . . . 42

2.3.2 Results of Annotations on Digital Libraries . . . . . . . . . 45

2.4 Gamification . . . . . . . . . . . . . . . . . 47

2.4 .1 Defining Gamification . . . . . . . . . . . . . . 47

2.4 .2 Does Gamification Work? . . . . . . . . . . . . . . . . . 47

2.4.3 Implementing Effective and Meaningful Gamification . . . 48

2.4.4 Criticisms and Possible Pitfalls of Gamification . . . . . . . 51

2.5 Gamification Within Digital Libraries . . . . . . . . . 52

2.6 Annotation Data Model Specifications . . . . . . . . . . 53

2.6.1 Open Annotation Data Model . . . . . . . . . . . . . . . . 53

2.6.2 Web Annotation Data Model . . . . . . . . . . . . . . . 55

2.7 Summary . . . . . . . . . . . . . . . . . 57 


\subsection{Overview}

This section revisits various projects and research that have investigated, analysed or implemented annotation or gamification concepts: giving a brief overview of what they entailed, their conclusions or summaries and any possible future work highlighted by the authors. We first look at annotation and gamification separately and thereafter relevant studies carried out on how to enhance digital heritage archives.

Section 2.2 covers different aspects and implementations of annotation frameworks in the past few decades. While there are probably more annotation initiatives than those covered, the selected projects are carefully chosen to illustrate clear ideological breakpoints when it comes to how possible solutions were perceived through a definitive timeline. Section 2.3 reviews the use of annotations in four notable digital heritage library projects and also aggregates their effects.

Section 2.4 introduces the concept of gamification, reviews if gamification works based on proven human motivation theories, defines the criteria used by the project to determine effective and meaningful gamification and also highlights some criticisms and possible pitfalls of gamification. Section 2.5 then looks into the use of gamification in digital libraries.

Finally, section 2.6 covers the two Annotation Data Models currently available, namely: the Open Annotation Data Model and the Web Annotation Data Model.

\subsection{Annotations and Annotation Frameworks}

\subsubsection{A Model for Annotations}

The traditional definition of annotations refers to notes or markings made on paper, books or other physical documents [67]. In the digital age, particularly digital libraries, this term has had to evolve from simple content types such as text, to more complex media types such as 3D objects [55]. This research focuses on annotations that are Web-based (available via Web interface) and collaborative (enable users to share annotations).

Agosti and Ferro's work provides a good foundation for a formal model for the annotation of digital contents [21]. They categorise annotations into two groups based on the approach used: either as metadata annotations or as content annotations.

For the former, annotations are considered as additional data that is associated to the content and at the same time defines its attributes and structure thus giving it semantic meaning. In this approach, the annotations have to follow a 
specification that defines the format and values that the annotation should have so that the data can be both readable by humans and by computers [21]. This reflects a data specific view of the annotation [22]. The advantage of this is that, while these annotations are easily readable by human beings, the computers are also able to use that data interchangeably across different systems to provide extra functionality or value.

On the other hand, we have content annotations. These are viewed as related additions to the existing content that form a layer where ideas are clarified, explained or discussed further. Unlike the previous approach, this is not achieved through the formal structure of the annotation (its semantic value) but rather through the content of the annotation itself. It is the additional content that helps others understand the annotated content. These annotations therefore are intended for human recipients as the annotated document is not made more readable to computers than it was before annotation [21]. This reflects an information specific view of the annotation [22].

Annotations have come a long way over the past two decades. In this time, there have been some annotation frameworks built that fall into these two broad categories to address different problems as the designers saw fit. The first attempts at annotation on the Web seemed to focus on adding text annotations only to structured documents [56]. Once this was achieved, annotation support was then extended to other more complex media types such as images, video, audio and even 3D objects [87]. Some initiatives sought to annotate these multimedia type documents with objects that are themselves of multimedia nature [33]. Others focused less on support of different media types and more on the structural aspects of the annotation frameworks, such as how they could reliably archive the annotations over time [81].

\subsubsection{Major Annotations Frameworks}

\section{Annotea}

The Annotea Project was an effort by the World Wide Web Consortium ${ }^{1}$ (W3C), started in 2001, to create a standard for shared Web annotations and is but one of the group's efforts towards a Semantic $\mathrm{Web}^{2}$ [58].

It is Web-based and makes use of the Resource Description Framework (RDF) infrastructure, which allows annotations to be objects that have inherent semantic value [14]. The metadata generated from the annotations therefore is not only used in describing the content but may also provide the means to organise it

\footnotetext{
${ }^{1}$ http://www.w3.org/Consortium/

${ }^{2}$ http: //www .w3.org/standards/semanticweb/
} 
further and even power extra functionality such as a more refined search and linking of annotations to related objects [56,58].

Annotea makes use of key existing W3C specifications as much as possible. Examples include: RDF, XML Pointer Language ${ }^{3}$ (XPointer), XML Linking Language $^{4}$ (XLink) and the Hypertext Transfer Protocol ${ }^{5}$ (HTTP) [87].

The first phase of Annotea introduced Web annotations and it allowed the user to reply to an annotation, thus forming a reply thread. The second phase introduced bookmarks and topics $[57,59,61]$.

\section{Vannotea}

Unlike the Annotea Project, which was limited to only annotate those resources that had structure (HTML or XML documents) and a Uniform Resource Identifier (URI) [56], the Vannotea Project supported annotation of fine-grained contexts within various multimedia objects [87]. It also included the combination of a number of open source technologies that powered its extra features, such as [86]: a) Jabber to provide instant messaging functionality [18]; b) Shibboleth to provide secure user authentication [19]; and c) eXtensible Access Control Markup Language (XACML) as the XML-based language used to define access control policies [6]. Despite its differences to Annotea in terms of functionality, it was still RDF-based and could be viewed as an extension of Annotea [87].

\section{Multimedia Digital Annotation System (MADCOW)}

So far, the common feature of efforts such as Annotea was that the user was expected to annotate using specialised browsers that had the annotation capabilities built in, such as the Amaya browser [4]. This often limited support and disrupted the user's navigation [33].

Contrary to this approach, the Multimedia Digital Annotation System (MAD$\mathrm{COW}$ ) was created as a plugin for a standard Web browser. The system relied on a client-server architecture where the plugin would serve as the client and the servers would be the stores from which annotations would be accessed by the client [33]. This was done so that the system could be integrated into different browsers and Web servers, thus being platform-independent [34].

\footnotetext{
${ }^{3}$ http://www.w3.org/TR/xptr-framework/

${ }^{4}$ http://www.w3.org/TR/xlink/

${ }^{5}$ http://www.w3.org/Protocols/
} 


\section{LEMO}

The LEMO Annotation Framework was a uniform, multimedia-enabled annotation framework that went beyond the state of the art annotation software at the time. This was due to the intentional effort to adapt linked-data principles, thus treating annotations as first-class Web resources that could be linked to one other [51]. The LEMO framework was to be:

- Linkable - Annotations were first class objects and thus able to be referenced since they had their own Uniform Resource Locators (URLs).

- Extensible - The system was able to support various types of annotations (despite having different structures) and content (for example, PDF files) without changes in the core components.

- Multimedia-enabled - The system included support for multimedia objects.

- Open and Interoperable - The annotations were to be available on the Web and thus accessible from other applications (where applicable).

Just like the Vannotea Project, LEMO borrowed the core design from the Annotea Project but with some extended functionality to adapt to the linked-data principles [51, 83]. Existing as a separate repository, LEMO provided a REST 6 interface to allow fetching, creating, editing and deleting of annotations.

The goal of this project was to coalesce three fundamental attributes into one solution, whereas the other previous projects mostly focused on one or two [51]. The first is a uniform annotation model for multimedia contents and various types of annotations. Second is to be able to address fragments of various content types in a uniform, interoperable manner. Third is to pull annotations out of closed data silos and make them interoperable.

\section{Memento}

Van de Sompel et al. proposed a model that was an extension to HTTP for the archiving of Web content [93]. The goal of this model was to connect the original resource to the archived version of that resource to cater for the fact that a resource could change over time $[53,93]$.

This model was applied to annotations in the Memento framework. The main differentiator of this framework from its predecessors was the inclusion of an intermediary server that would serve to create archived versions of the resources

\footnotetext{
${ }^{6}$ Representational State Transfer
} 
so that a representative version of a resource could be requested when required [81]. The ultimate objective was to provide a way for old annotations to remain relevant even if the original resource was edited over time [52].

\section{Annotator}

Annotator is an annotation framework that comprises of two parts: Annotator JS as the client-side component and AnnotateIt Store ${ }^{8}$ as the server-side component [89].

Annotator JS (client-side) is an open source ${ }^{9}$ JavaScript library for annotation with a set of plugins that can be used to easily add extra annotation functionality to any Web page [7]. This modular plugin architecture makes it easy to add any required out-of-core functionality.

The default plugins ${ }^{10}$ are developed and maintained by contributors to the core and are considered part of Annotator. On the other hand, third party plugin ${ }^{11}$ have been created by the members of the community to extend Annotator beyond text annotations to other types of media such as images, videos and PDFs. Some of the default plugins and their descriptions are listed in Table 2.1.

Annotatelt Store (server-side) is an open source ${ }^{12}$ storage API and a reference implementation of a backend store to persist annotations created by Annotator JS [89].

Unlike Annotator JS, it is designed to run on a server that may service several clients (presumably running Annotator JS). Annotator JS only handles the user interaction, presentation and website integration aspects of Annotator and therefore, if used alone, the user would lose all annotations made on that page on refresh. When used together, Annotator JS is able to serialise the data representing the annotations and send it to the AnnotateIt Store, which authenticates the user and persists the annotations in a database.

\subsubsection{Comparison of Annotation Frameworks}

While Annotea, Vannotea, MADCOW, LEMO, Memento and Annotator do not cover all the annotation frameworks ever created in the past few decades, they do show major differences in infrastructural design. These are highlighted in subsection 2.2.2.

\footnotetext{
${ }^{7}$ http://annotatorjs.org

${ }^{8}$ http://annotateit.org

${ }^{9}$ https://github.com/openannotation/annotator

${ }^{10}$ http: //annotator.readthedocs.org/en/latest/plugins/index.html

${ }^{11}$ https://github.com/openannotation/annotator/wiki\#plugins-3rd-party

${ }^{12}$ https://github.com/openannotation/annotator-store
} 


\begin{tabular}{l|l} 
Plugin & Description \\
\hline Auth & $\begin{array}{l}\text { Provides an authorisation mechanism to authenticate } \\
\text { annotations created by users. It authenticates against } \\
\text { the annotation data store that is provided via the store } \\
\text { plugin. } \\
\text { Adds a toolbar at the top of the page that enables fil- } \\
\text { tering and navigation of annotations displayed on the } \\
\text { Furrent annotated page. }\end{array}$ \\
Markdown & $\begin{array}{l}\text { Enables use of Markdown syntax in the annotation to } \\
\text { provide minimal HTML formatting options. }\end{array}$ \\
Permissions & $\begin{array}{l}\text { Enables setting of access levels to control who should } \\
\text { be able to view, edit and delete the annotations. } \\
\text { Serialises and sends the annotation data to a server } \\
\text { during key events triggered by the annotator. }\end{array}$ \\
Store & $\begin{array}{l}\text { Adds the ability of view and attach keywords to anno- } \\
\text { tations. } \\
\text { Trovides a notification to users who are using unsup- } \\
\text { ported browsers. }\end{array}$ \\
Unsupported
\end{tabular}

Table 2.1: Default Annotator JS plugins

Despite these differences, there are some key similarities, especially if evaluated from a functional point of view $[7,33,51,56,85]$ :

- Access control - There was an element of privacy in each framework, however, the implementation varied slightly. For example, in Annotea, there were local (private) and remote (shared) annotations. Local annotations were stored on the user's host computer while the remote annotations were assumed to be publicly viewable. In Annotator, access control was more finegrained. All annotations were stored on the server. The user then had the ability to set the permissions on who should be able to view, edit or manage the annotations.

- Annotations as first class Web resources - Each annotation was associated with a URI. This made it possible to reference them individually.

- Interoperability - There was a focus on a design that would allow different applications to be able to exchange information. Annotea's choice of RDF as a format was partly motivated by the fact that other tools could then easily understand and make use of its structure (being a well defined specification). LEMO and Annotator encouraged interoperability by providing REST interfaces. 
- Multiple annotation servers - Annotea had multiple servers to cater for scalability and privacy problems. Different user groups were expected to set up their own servers where they would be able to control access. This model was closely followed by the other frameworks.

- Open technologies - All these frameworks were built on open standards to simplify interoperability and maximise extensibility, for example, HTTP. Annotea used RDF, XPointer and XLink. Vannotea and LEMO also made use of RDF. Annotator, on the other hand, made use of JSON as the preferred communication format.

- Switch between navigation and annotation modes - Included were annotation tools that would support creating, editing, updating and deleting annotations. Effort was made to make these tools easy to integrate in existing browsers while allowing the user to switch easily between the navigation and annotation modes.

- Visualisation of placeholders for annotations - Different frameworks chose different ways to display single and threaded annotations. Annotea chose to use small icons to show where the annotations were placed. On click, the annotation would then be displayed in a popup window. Other tools, like Annotator, preferred highlighting the annotation in a different colour to better distinguish from the fixed content.

\subsubsection{Motivations for Annotation}

In Marshall's study on annotations made on paper, she highlights that annotations are evidence of human attention and that the attention of the person annotating may be applied to different tasks, for example to [67]:

i) serve as a procedural marker (anticipate for future attention)

ii) serve as an aid to memory

iii) be part of the problem working process

iv) show interpretive reading

v) trace the reader's attention.

This shows that annotation is a cognitive process and it cannot be done without the annotator being engaged with the content being annotated.

When applied to digital systems, most users have one or two main motivations for tagging. A study by Ames and Naaman breaks down annotation motivation into two dimensions: sociality and functional [28]. 
The first dimension (sociality) relates to the intended usage with respect to other users while the second dimension (functionality) relates to the intended usage with respect to how it enables certain features or tasks [28]. See Table 2.2.

\begin{tabular}{|l|l|l|}
\cline { 3 - 4 } \multicolumn{2}{c|}{} & \multicolumn{2}{c|}{ Function } \\
\cline { 3 - 4 } \multicolumn{4}{c|}{ Sociality } & \multirow{2}{*}{ Self } & $\begin{array}{l}\text { Organisation } \\
\text { Retrieval, Directory }\end{array}$ & $\begin{array}{l}\text { Communication } \\
\text { Search }\end{array}$ \\
\cline { 2 - 4 } & Social & $\begin{array}{l}\text { Contribut for self } \\
\text { Ad hoc photo pooling }\end{array}$ \\
\hline
\end{tabular}

Table 2.2: Taxonomy of tagging motivations from a study using ZoneTag/Flickr (a photo tagging application) [28].

ZoneTag/Flickr was the photo application developed for the aforementioned study. A brief explanation of each section in Table 2.2 follows below [28]:

- Self I Organization - represented the traditional annotation motivations found in personal photo collections. Some participants tagged so as to be able to retrieve photos for sharing while others tagged for their own organisational purposes.

- Self I Communication - was primarily used to aid in adding context to a photograph such as the name of the place that the photograph was taken at or the names of people in the photograph.

- Social I Organization - represented the participant's motivations for making their photos findable by specific people or strangers.

- Social I Communication - was used to communicate contextual information to others.

Another study by Nov and Ye supported these findings showing that both social presence and individual level motivations affect a user's tagging habits and also that social presence has a larger influence [74].

It should be noted that both studies focused only on Flickr, which may have limited the applicability of the findings in a broader context.

\subsection{Annotations Within Digital Libraries}

The definition of what a digital library is varies with context. The two main schools of thought come from the research oriented community and the traditional library community [47]. The former views a digital library as a system that 
is concerned with the creation, management and movement of information by a selected user group. The latter views digital libraries as institutions or organisations that provide information in digital form.

Agosti et al. highlight distinguishing features from these definitions, which are creation and effective use [22]. Digital libraries therefore are characterised by the collection of information and the community of users to whom the collection caters. Other aspects include the interpretation of and collaboration around these resources [22].

Annotations on digital content can be used to realise these distinguishing features [22] without the limitations that exist with annotations on books. An example of one limitation is the impracticality of annotating on a book that should be shared by many people [67]. Collaboratories, on the other hand, enable people who are spread out geographically to function in virtual teams that work together as if they were in the same space [92].

With tools such as annotations integrated into digital libraries, the way users engage with them is changing. Users now directly work with the tools the digital library systems provide to merge their contributions with the information resource. Therefore the digital library is not merely a tool that users consult for information but a key part of the intellectual process [20].

Even though user generated annotations generate index terms of lower accuracy than those generated by the domain expert, Nichols et al. believe that the digital library is made better with them included [72].

\subsubsection{Examples of Digital Libraries With Annotations}

Some of the notable digital libraries that implemented annotations are DEBORA, COLLATE, DiLAS and CULTURA. Each had a different focus and use-case, all of which are highlighted below:

\section{DEBORA}

DEBORA (Digital Access to Books of the Renaissance) aimed to explore the possibility of incorporating collaborative features into a digital version of Renaissance books [43, 72]. One of the proposed approaches for users to share ideas within DEBORA was through a personal annotation facility but without a model for discussion $[35,45]$.

As part of the design process, Nichols et al. studied the current collaborative practices on the Renaissance texts at the time as a way to make sure that they came up with a system that was complementary to already existing practices [72].

They first recognised that there is potential for users to contribute to a digital collection through User Supplied Data (USD). Then they categorised USD types 
into two main categories [72]:

- Implicit - data generated by the user's activity, such as: i) search term queries and $i$ ) ratings .

- Explicit - data that the user explicitly generates, such as: i) annotations, ii) key-word additions, iii) evaluative commentary, iv) hypertext links, v) ratings and vi) error correction .

Nichols et al. found that the annotation feature was appreciated by the users and seen as a key tool to foster collaboration among users as intended [72].

\section{COLLATE}

The goal of COLLATE (Collaboratory for Annotation Indexing and Retrieval of Digitized Historical Archive Material) was to develop a cultural collaboratory to support interpretive work [23]. The body of work that this system was built to support was the digitised records of European films of the $20^{\text {th }}$ century. The system would collect contributions from individuals and domain experts who would then analyse, evaluate, index and annotate to provide context in the library [92].

Initially, the project architecture favoured the task-oriented approach to annotations but then shifted to a discourse-oriented approach due to their analysis of user behaviour. From the analysis they realised that annotation in some cases cannot be split into different tasks between different users [45]. This was a different approach to the DEBORA project, which did not even model for discourse $[35,45]$.

\section{DiLAS}

The objective of DiLAS (Digital Library Annotation Service) was to design and develop a generic annotation service that could be used in Digital Library Management Systems (DLMSs). Agosti et al. defined some of the use cases that reflect the functionalities that an annotation tool might offer to a user [23, 24]:

a) User-level Use Cases - data generated by the user's activity, such as:

- Create annotations - Users should be able to create annotations and not only annotate one kind of object but different kinds of objects.

- Modify and delete annotations - Users should be able to update or delete any annotations that they've made.

- Set the scope of annotations - Users should be able to define whether their annotation is publicly available or private. 
- List annotations - Users should be able to view all annotations made on an associated resource that is in view.

- Browse annotations - In the case of nested or linked annotations, the user should be able to browse or view all the relevant annotations.

- Search and retrieve annotations - Annotations should themselves be part of the search system and therefore searchable.

b) System-level Use Cases - data that the user explicitly generates, such as:

- Group and user management - Support a user model that would be used to power the ability to scope the annotation to a particular user or group.

- Create and store annotations - Ability for the system to persist the annotations once the user creates them using the interface and include metadata about the annotations. The metadata would then be used to associate the annotations with the annotation resource and the author of the annotations.

- Delete and modify annotations - Support ability for deletion of annotations once created and perform the right permission checks to make sure only authorised users are allowed to do so.

- Set scope of the annotations - Have the ability to store the permissions associated with the annotations to enable the user to have public or private annotations.

- Query and fetch the annotations - The system should have the ability to query for annotations connected to a specific resource. This resource could be one object or even a page on the digital library.

\section{The CULTURA Project}

CULTURA $^{13}$ (Cultivating Understanding and Research through Adaptivity) aimed to provide adaptive and interactive user environments that allowed for the investigation, comprehension, contribution to and enrichment of digital humanities artefacts and collections [5].

The project was made up of two major artefacts: the 1641 Depositions $^{14}$ and the IPSA (Imaginum Patavinae Scientiae Archivum) Digital Herbal Archive ${ }^{15}$. These formed the test bed for their hypotheses. The 1641 Depositions are a collection

\footnotetext{
${ }^{13}$ http://www . cultura-strep.eu

${ }^{14}$ http://1641.tcd.ie/

${ }^{15}$ http://ipsa.dei.unipd.it
} 
of witness testimonies by Protestants and Catholics concerning their experiences of the 1641 Irish rebellion [1] while the IPSA Digital Herbal Archive is an online digital heritage archive that gathers images and texts from ancient manuscripts and has a focus on botany, astrology and medicine [2]. These collections were selected so as to have two different media formats on which they could test some of the key technologies. The former targeted text and the latter targeted images [26].

One of the objectives of CULTURA was to bridge the gap between the expert users, who were knowledgable on the relevant search parameters to access the digital content and the other users who had to be guided through good user interface and intuitive design in order to retrieve the content $[26,49,50]$.

\subsubsection{Results of Annotations on Digital Libraries}

\section{Collaboration and Discourse}

Annotations allow new ideas and concepts that arise from the discussion around the content to be integrated into a newly created resource [22]. Users could even come together in groups and contribute to collections instead of being confined to only reading or viewing [72].

COLLATE took this idea further as it took into account users' roles, tasks and goals while modelling a collaborative system. The users came from different backgrounds and contexts and the domain objects represented the main body of work. This enabled them to have context-based indexing that created value added information that could form a thread of information, much like a discussion around a particular topic [92].

The result of all these interactions between users is that a communication channel is formed through the annotations [25].

\section{Context Around Content}

In systems where users provide extra information via annotations, we find that the annotations provide additional context about the annotated content [22]. Users may also use annotations to provide alternative points of view and highlight content that needs to be discussed further [25].

\section{User-generated Object Metadata}

The pooling together of multiple annotations from several sources has the effect of significantly increasing the metadata available on a particular topic or object of interest [60]. 
For example, in COLLATE, applying the right knowledge management tools such as indexing aids and domain-specific controlled vocabularies can create a growing body of metadata [92]. This user-generated metadata can then be used to provide semi-automatic categorisation and even possibly infer relationships between the objects. In DEBORA, a simple interface was included to enable authorisation of metadata supplied by users [72].

\section{Ability To Provide Smarter Search}

Annotations make it possible to better determine the relationships between documents using the extra information gathered from multiple annotations [25]. Search is considered a basic user requirement in digital collections [91]. To allow for advanced content and context based search, the documents in the collection should be indexed and organised in a reasonable way [92].

Annotations allow linking together of content to provide alternative navigation and improve browsing capabilities. Agosti and Ferro presented a system that used annotations as context for searching documents [20]. This resulted in a search strategy that took advantage of the annotations to improve the results of the search by ranking them based on relevance.

\section{Improved Engagement}

In CULTURA, users were able to add annotations and notes [27]. These enabled users in different levels to engage with the content in different ways. Art historians were able to view extra information such as pictorial techniques and iconography, which they could then use to recognise the authorship of the artist. Professional researchers in turn highlighted details that were relevant to their research and professors would then use the information to teach their students more about how art historians work with art [27, 49].

\section{Personalisation}

Annotation offers personalisation depending on the knowledge level of the user. A user evaluation carried out on the CULTURA environment revealed that professionals who were well versed in the cultural collection were less likely to utilise recommendations when compared to researchers learning about the collections [49]. However, annotations and bookmarks were found to be more useful to the professional [31, 49]. 


\subsection{Gamification}

\subsubsection{Defining Gamification}

Gamification is defined as the use of game design elements in non-gaming contexts to motivate users, increase user-activity and user-retention [41, 42]. It is often implemented as a service layer of reward and reputation systems with points, badges, levels, status, progression bars and leader boards [41, 70, 97]. The reward and reputation sometimes depends on community feedback from the system $[41,97]$.

Deterding et al. sought to break down this definition into its constituents, which are: game design, elements and non-game-contexts [41]. These are explained below:

1. Game Design - Refers to designing for gamefulness, where gamefulness refers to the experiential and behavioural qualities that can make the system that it is being applied to somewhat playful.

2. Elements - Whereas serious games are full-fledged games for purposes outside entertainment, the term elements here shows that gamification involves incorporating only some design characteristics of a game into a gamified application.

3. Non-game Contexts - These refer to situations or circumstances outside their expected use case, which would be entertainment in a full-fledged game.

\subsubsection{Does Gamification Work?}

There are many types of game design elements. Examples include: i) points; ii) badges; iii) leaderboards; iv) progress bars; $v$ ) performance graphs; vi) avatars; vii) profile development; viii) quests; and ix) meaningful stories [80]. However, points, leaderboards and achievements are the most frequently referenced and studied [48, 77].

In a gamified system, points are virtual rewards given to a user based on a predetermined action, badges are visual representations of a user's achievements and leaderboards provide ranking of users based on a certain performance criteria $[80,96]$.

These game design elements are known to provide extrinsic motivation in non-game contexts [62]. Gamification seeks to take advantage of this fact by using extrinsic motivations to evoke intrinsic motivations [71]. In Intrinsic motivation, 
users carry out an activity for its internal and personal value while, in extrinsic motivation, they carry out an activity due to an external trigger or reason [38, 39].

Users of gamified systems find each game design element rewarding depending on their intrinsic needs, values or goals [77]. However, Sailer et al. point out that we should consider the possibility that user interaction with multiple game design elements can have varying and complex motivational effects [80].

In Table 2.3, Richter et al. propose a framework to classify human motivations to game elements and their intended objectives [77]. Understanding the right motivation driver is essential to designing an effective gamification system that fulfils the intended objectives.

A study carried out by Mekler et al. evaluated the effects of the three game design elements i.e. points, levels and leaderboards [70]. They found out that they increased performance but not did not affect intrinsic motivation nor destroy it. They advised that these game design elements, though easy to implement and effective, have effects that only last for the short-term and therefore should not be used to sustain long-term engagement with the user.

The study by Hamari et al. took a different approach [48]. They investigated the effectiveness of gamification by studying peer-reviewed empirical studies of gamification instead of carrying out their own gamification experiment. They found out that the majority of studies that were reviewed showed that gamification bore positive results. However, they observed that results from quantitative studies varied from those results from qualitative studies. For the former, there was a direct relationship between the gamification elements and the results. For the latter, the observation was that there are several factors that may affect the results. They suggest these factors to be the role of the context being gamified and personal qualities of the users.

\subsubsection{Implementing Effective and Meaningful Gamification}

The game design elements used as components of a gamified system have to contribute to meaning because a user will consider an activity or task to be meaningful if it is considered valuable by the user [69].

In this regard, gamification has the potential to foster motivation in different contexts of the user. Sailer et al. list six principal perspectives from motivational research that can be associated with game design elements and thus should be considered when motivating gamification environments [80]:

1. Trait Perspective - Motivation here depends on the individual characteristics of the user. For example users with a strong achievement motive are more motivated if gamification emphasises achievement, success and 


\begin{tabular}{|c|c|c|}
\hline Motivation theory & Incentives/rewards & Role \\
\hline \multirow[t]{2}{*}{ Self efficacy } & $\begin{array}{l}\text { Audio/verbal/visual/ } \\
\text { music/sounds effect }\end{array}$ & Feedback \\
\hline & Progress bar & Feedback, achievements \\
\hline \multirow[t]{2}{*}{$\begin{array}{l}\text { Self-efficacy, goal-setting, } \\
\text { personal investment, } \\
\text { expectancy value, } \\
\text { need achievement }\end{array}$} & Points/bonus/divident & $\begin{array}{l}\text { Feedback, reward, status, } \\
\text { achievements, } \\
\text { competition, progression, } \\
\text { ownership }\end{array}$ \\
\hline & $\begin{array}{l}\text { Mini games/challenges/ } \\
\text { quests }\end{array}$ & $\begin{array}{l}\text { Reward, status, competi- } \\
\text { tion, achievements }\end{array}$ \\
\hline \multirow[t]{4}{*}{$\begin{array}{l}\text { Self-efficacy, goal-setting, } \\
\text { personal investment, } \\
\text { expectancy value, } \\
\text { social comparison }\end{array}$} & Badges & $\begin{array}{l}\text { Status and reputation, } \\
\text { achievements and past ac- } \\
\text { complishments, collection, } \\
\text { competition, ownership }\end{array}$ \\
\hline & Virtual goods & $\begin{array}{l}\text { Reward, social, status, } \\
\text { achievements, ownership, } \\
\text { self-expression }\end{array}$ \\
\hline & Leaderboard & $\begin{array}{l}\text { Status and reputation, } \\
\text { achievements, competition }\end{array}$ \\
\hline & $\begin{array}{l}\text { Rewards-choosing colors, } \\
\text { power }\end{array}$ & Achievements \\
\hline \multirow{2}{*}{$\begin{array}{l}\text { Self-efficacy, goal-setting, } \\
\text { personal investment, } \\
\text { expectancy value, } \\
\text { need achievement, } \\
\text { social comparison }\end{array}$} & Achievements & $\begin{array}{l}\text { Collection, status, } \\
\text { competition, discovering, } \\
\text { progression }\end{array}$ \\
\hline & Levels & $\begin{array}{l}\text { Feedback, status and repu- } \\
\text { tation, achievements, com- } \\
\text { petition, moderate chal- } \\
\text { lenge }\end{array}$ \\
\hline $\begin{array}{l}\text { Social comparison, } \\
\text { personal investment, } \\
\text { expectancy value }\end{array}$ & Avatar & $\begin{array}{l}\text { Social, self-expression, } \\
\text { ownership }\end{array}$ \\
\hline
\end{tabular}

Table 2.3: Theoretical base of incentives and rewards by Richter, Raban, and Rafaeli [77].

progress. Those with a strong affiliation motive would be more motivated if gamification emphasises membership.

2. Behaviourist Learning Perspective - Motivation results from positive and negative feedback. For example, a user may be more motivated if gamification provides feedback or rewards of some kind.

3. Cognitive Perspective - Defines motivation as the result of a means-to-an- 
end analysis by the user. For example, a user would likely be motivated if gamification provided a clear and achievable goal or highlights the resulting consequences of a goal.

4. Perspective of Self-Determination - Presents motivation as a factor of psychological needs. For example, users are more likely to be motivated if they feel competent, autonomous and socially related.

5. Perspective of Interest - This takes into account individual preferences and content aspects. For example, a user is more likely to be motivated if they are interested in the subject matter or if the level of difficulty adapts to their skills and competencies.

6. Perspective of Emotion - This focuses on the emotions of the user. For example, a user is more likely to be motivated if gamification increases positive feelings or reduces negative feelings.

Therefore, meaningful gamification should, at its core, have the needs of the user over the needs of the body of work within which gamification is being implemented. It should be an experience that is well-connected to the underlying non-game context to allow them to have a positive internalised experience [73].

Aparicio, Vela, Sánchez, and Montes propose a method based on the selfdeterministic perspective mentioned in Sailer et al.'s research as a way to come up with effective gamification [30,80]:

1. Identification of the main objective - This is the task that will be gamified and should be clearly defined from the beginning.

2. Identification of the traversal objective - This is the underlying objective that will be interesting to the participants.

3. Selection of game mechanics - These would be a selection of gamification methods that match the objectives and support the needs of human motivation, which are:

(a) Autonomy - Refers to the desire for an individual to have a sense of ownership, control their own actions and how they perform a task. Examples are: profiles, avatars, macros, configurable interfaces, alternative activities, privacy controls and notification controls.

(b) Competence - Refers to the need of people to feel competent and efficient when participating in challenges. Examples are: positive feedback, optimal challenges, progressive information, intuitive controls, points, levels and leader boards. 
(c) Relatedness - Refers to the need for an individual to feel connected to others. Examples are: groups, messages, blogs, connection to social networks and chat.

4. Analysis of the effectiveness - Analysis of the effectiveness of the implementation of gamification based on fun, quality indicators and satisfaction, and service quality.

In the end, the application of gamification and its effects should not be obvious to the users as the idea is to create an impression of intrinsic motivation [30]. Intrinsic motivated activities are those that the individual finds interesting and performs without any kind of conditioning, just by the mere pleasure of carrying them out while extrinsic motivated activities are those activities that are carried out to achieve a certain outcome $[38,39,79]$.

\subsubsection{Criticisms and Possible Pitfalls of Gamification}

Gamification has not alway been cast in a positive light and, in fact, some have offered criticism of either the concept itself or the implementation [32, 68, 78, 95]. It is prudent and important to have these in mind so as to be mindful of the possible pitfalls when applying gamification to any system.

Previous studies have shown that extrinsic rewards sometimes have negative effects on intrinsic motivation even when offered as indicators of positive actions $[38,39]$. Some game designers argue that the current implementation of gamification by using points badges and leaderboards places the emphasis on the least essential part of games. The bone of contention here is that the pleasure of a game is not from these gamification elements but from the careful design of the goals in their respective context [40].

Others have had more subtle issues with gamification, stating that by putting the term game first, it implies that the entire activity will be somewhat close to a full-fledged game or confused for one [73]. This has been deemed misleading as to the true intentions of applying gamification versus its real-world effect.

Some argue that pointsification should be a more appropriate term for systems that add nothing more than a scoring system to a non-game activity [78]. One of the harshest criticisms is by Bogost, who says that gamification is primarily a practice of marketers and consultants who seek to construct and then exploit an opportunity for their benefit [32]. The opportunity he is speaking of is when gamification is offered as a solution to a traditional business that sometimes does not understand games.

Gamification should not be seen as a one-size-fits-all solution for improving user engagement, motivation or participation. The criticisms previously high- 
lighted come from researchers who observed the abuse of gamification by applying it in certain contexts without thinking through the intended objectives (mentioned in section 2.4.3). It is therefore imperative that in our application of gamification the focus is kept on the core activity. The purpose of gamification is to amplify intrinsic value [40]. Understanding what the users desire without gamification (in terms of value) goes a long way to understand what kind of experience one should create and what game design elements one should incorporate.

\subsection{Gamification Within Digital Libraries}

Galleries, Libraries, Archives and Museums (GLAMs) have been exploring crowdsourcing as a way to build up or complement digital heritage libraries. However, one of the major challenges with this is gathering loyal users [75]. One approach that has been used to tackle this problem is Games With A Purpose (GWAPs). GWAPs provide an avenue for people to perform tasks that computers are unable to perform as a side effect of playing games [94].

GWAPs should not be confused with gamification. While GWAPs focus on making the work fun, the focus of gamification is to incentivise the user's actions. Therefore it's imperative to look at previous attempts to apply gamification to digital libraries (especially those concerned with cultural and heritage content).

Mekler, Brühlmann, Opwis, and Tuch carried out a study that investigated the relationship between game design elements and meaning [69]. The experiment they carried out involved 172 users participating in an image annotation activity. The users were differentiated using two main criteria: i) points vs. no points and ii) meaningful framing vs. no meaningful framing. They found out that:

1. Points motivated the participants to create more tags. They attributed this to the ability of points to provide immediate feedback to the participant and have that feedback linked to their own performance.

2. Meaningful framing motivated the participants to do a better job with their annotations. The participants would spend more time while making each tag.

3. Both points and meaningful framing on their own increased the participant's intrinsic motivation to the same degree.

4. The combination of points and meaningful framing resulted in the highest levels of the participant's intrinsic motivation.

5. Only the lack of both points and meaningful framing resulted in a lower level of intrinsic motivation. 
Other attempts at gamifying digital heritage archives include work done by Schlötterer et al., which was based on Europeana ${ }^{16}$ [84]. They implemented a question and answering game where the users of the system would get to answer questions asked by other users. The former group of users were expected to use a search interface to query for answers while the latter group of users got to ask questions but had to provide the correct answer as a URL. If the answer was correct the user would be awarded experience points and the answer (provided as a URL) would be deemed correct. High score lists indicated the top player and other registered players could review how the questions were answered.

Their results showed that all participants liked their gamification implementation and design [84]. Some users requested more reward elements, the ability to see more about how other users found the correct results and even access to their own gaming history so that they can assess their gameplay for future improvement.

The rationale for their gamification approach was that it would give playful access to cultural content [84]. The system and data generated would in turn facilitate: $i$ ) the distribution of cultural content; $i$ ) the identifying of interesting content; and iii) the collection of data to support user queries .

For both studies $[69,84]$, it seems that gamification was received positively by the participants.

\subsection{Annotation Data Model Specifications}

\subsubsection{Open Annotation Data Model}

The Open Annotation Data Model is an initiative of the Open Annotation Community (OAC) Group, a working group of the W3C, whose purpose is to work towards a common, RDF-based, specification for annotating digital resources [13].

The OAC came up with the Open Annotation Data Model Specification to provide a standard description mechanism for sharing annotations between systems [52]. It touches on the core ideas on the structure of annotations without demanding structural changes of the platform that wishes to implement them. It is also designed to cover as many annotation use cases as possible.

Their effort started by working towards a reconciliation of two proposals that had emerged from around 2011: the Annotation Ontology ${ }^{17}$ and the Open Annotation $\mathrm{Model}^{18}$. Their final deliverable was a specification, published under an

\footnotetext{
${ }^{16}$ The Europeana Collections Ports is an interface to digitised artefacts from Europe such as films, books, paintings etc. See http://europeana.eu/portal/

${ }^{17}$ https : //code.google.com/p/annotation-ontology/

${ }^{18} \mathrm{http}$ ://www . openannotation.org/spec/core/20130208/index.html
} 
appropriate open license, informed by the aforementioned proposals and community feedback [13].

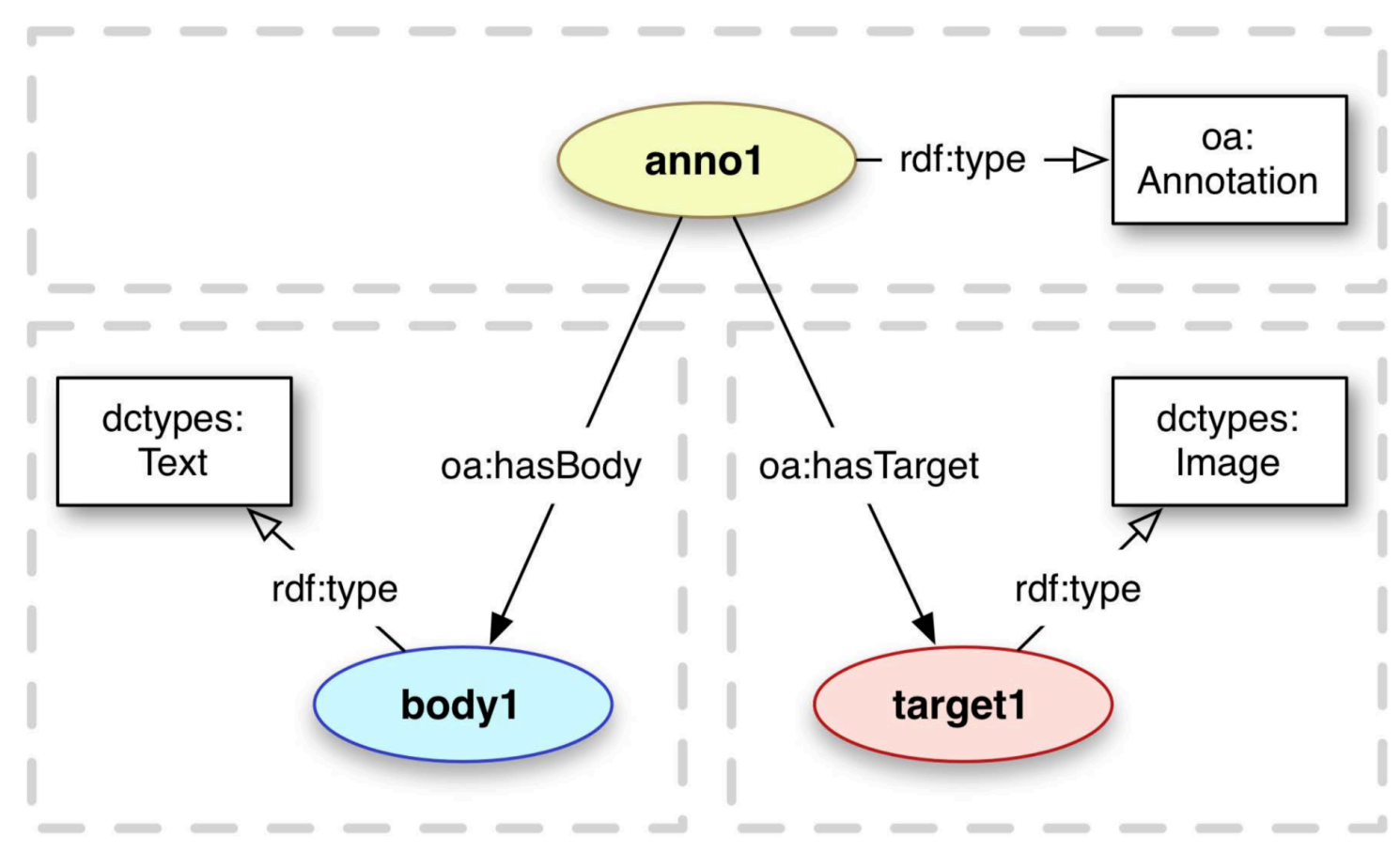

Figure 2.1: Illustration of the basic annotation data model by Sanderson, Ciccarese, and Van de Sompel [82].

As shown in Figure 2.1, the data model describes the relationship of two or more resources via an $\mathrm{RDF}^{19}$ graph. The annotation itself is made up of two parts: the Body and the Target [52, 82]. Both can be of different media types and serve different functions. The Body of an annotation usually has content that provides extra information about the Target while the Target is the resource that is being annotated.

The basic model can be extended with other attributes to: i) embed other resources; ii) include the annotator's motivations while making the annotation; iii) include semantic tags; and iv) even link to a specific part of a larger resource using fragment URIs [82].

\footnotetext{
${ }^{19}$ Resource Description Framework
} 


\subsubsection{Web Annotation Data Model}

The Web Annotation Data Model $^{20}$ is a draft specification ${ }^{21}$ that provides a specific JSON format for the implementation of annotations based on a conceptual model laid out in the Open Annotation Data Model [17]. JSON-LD is used to serialise the Linked Data while maintaining JSON compatibility, preserving human readability and allowing flexibility to express almost any kind of real world object $[12,17]$.

The JSON object contains varying attributes depending on the intended purpose. For example, Listing 2.1 shows the JSON representation of an annotation that is linked to an image and contains HTML content in the body.

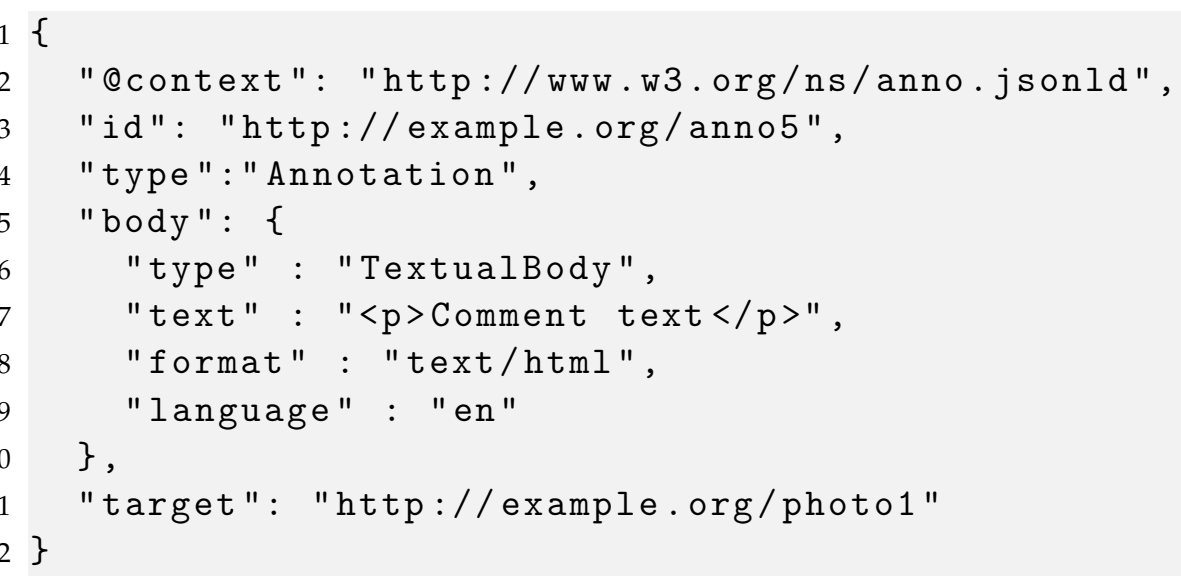

Listing 2.1: Example JSON representation of an annotation with image Target and an embedded textual body [17]

The model provides a standard that is adaptable to several use cases ranging from the simple to complex, while following Linked Data principles. Some of the considerations of the model include the ability to [17]:

- Reference an external Web resource - The Body and Target can reference a resource available at a URI and also include other information about the resource, such as its language and format.

- Have different classes for Web resources - In some cases it may be useful to know the type of context that a Body or Target references. This allows clients to intelligently determine the best way to download and render the

\footnotetext{
${ }^{20}$ http: //www .w3.org/TR/2016/WD-annotation-model-20160331/

${ }^{21}$ The latest draft of the specification was published on $31^{\text {st }}$ March 2016 and is still considered a work in progress.
} 
content. Examples of resources where this may be immediately applicable are image, audio and video files.

- Target segments of Web resources - URIs referencing Bodies or Targets do not have to reference the resource as a whole but may reference a section of the resource. The URI must include a fragment component that describes the section of the resource that is being referenced. However, it is important to note that not all media types support fragments and also that fragments vary depending on media type.

- Embedded textual bodies - Alternatively, one may need to add an annotation that contains information added into the Body and not reference an external Web resource. It is possible to include plain text into the Body as well as a representation of what would be in an external Web resource as long as the type and format are specified.

- Assign multiple Bodies and Targets - There is no fixed number for the number of Bodies or Targets that an annotation may have. It is possible to have a annotation without a Body or even have more than one Body. For example, when a user selects a block of text and highlights it, the visual result of that action is the annotation.

- Intelligent choice of Bodies and Targets - In some cases, one may have multiple bodies that have the same content but are served to the client depending on predefined criteria. For example, an annotation may include a comment in various languages and the Body is selected based on the language setting on the client.

- Attach extra contextual information about the annotation - Other than the details of the Body and Target, it is also possible to include other information about the annotation, such as:

- The author of the annotation.

- The client that was used when creating the annotation.

- The date-time that the annotation was created or had been last updated.

- The intended audience of the annotation.

- The motivation and purpose of the annotation.

- Any rights information to state under which conditions the resource may be used. 


\subsection{Summary}

So far we have shown that annotations have a come a long way over the past few years. They are now able to support complex implementations such as on audio and video, be applied to linked data principles and be treated as first class resources on the Web. The availability of open source JavaScript libraries and reference storage implementations have also made it possible to easily provide dynamic interfaces that users can use to create and manage annotations on digital heritage archives.

We looked into gamification, its definition, contributions to user motivation and how to best implement them by taking into consideration different factors, criticisms and possible pitfalls.

We also reviewed two Annotation Data Models and found that the Web Annotation Data Model is full featured and uses an easy to use data interchange format. This makes it a suitable guideline in our research experiments.

Lastly, for both annotations and gamification, we have investigated the effect that they would have on digital heritage archives separately. It is then probable that annotations put together with gamification could be the ideal candidate to improve user engagement and sharing across the different user groups of a digital heritage archive. It is within this frame that we make the case for gamified annotations. 



\section{Chapter 3}

\section{System Design and Implementation}

\section{Contents}

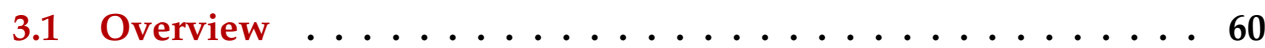

3.2 Establishing Requirements . . . . . . . . . . . . 60

3.3 Confirming Requirements . . . . . . . . . . . 62

3.4 The Digital Bleek and Lloyd Collection . . . . . . . . . . 64

3.4.1 The Source Data . . . . . . . . . . . . . . . . . . . . . . . . 64

3.4.2 Recreating the Archive for Testing . . . . . . . . . . . 65

3.5 System Overview . . . . . . . . . . . . . . . . . . 66

3.5.1 Interaction With Text Annotations . . . . . . . . . . . 67

3.5.2 Interaction With Image Annotations . . . . . . . . . . . 71

3.5 .3 Gamification Elements . . . . . . . . . . . . . . . . . 73

3.6 Building The Annotation Engine $\ldots \ldots \ldots \ldots$

3.6.1 API Endpoints (Server-side) . . . . . . . . . . . . . . 74

3.6.2 Annotator Library (Client-side) . . . . . . . . . . . . . . 80

3.7 Integrating Annotation Engine With The Digital Collection . . 83

3.8 Technologies and Tools Used . . . . . . . . . . . . . . . 85

3.9 Summary . . . . . . . . . . . . . . . . 86 


\subsection{Overview}

This chapter discusses the design and implementation of the systems developed for the purposes of this research.

Section 3.2 and section 3.3 document the process of establishing the confirming requirements of the system prior to the start of any work. Section 3.4 gives a brief description of the digital heritage archive that was used as the data source for this study - The Digital Bleek and Lloyd Collection.

Section 3.5 gives an overview of the the entire system and explains in detail the various interactions that the system provides to the viewers of the archive. Section 3.6 goes through the specifics of the server-side and client-side components of the annotation engine while section 3.7 explains how those components were integrated with the Digital Bleek and Lloyd Collection. Section 3.8 lists the main technologies and tools used to build and integrate the annotation engine.

\subsection{Establishing Requirements}

Requirements in this case refers to statements about the intended system that specify what it is, what it is expected to do or how it is expected to perform [88]. They are meant to be as specific, unambiguous and clear as possible, so as to make it easy to tell if they have been fulfilled in the developed system.

An exploratory study by Fu et al. investigated Web users' needs for personal annotation tools [46]. We used their work as a foundation to determine the basic requirements of an annotation system such as, the support for multiple media types, the ability to add/update annotations and the use Web-based annotations.

Additional requirements were extracted from the comparison of various annotation frameworks $[33,51,56,85]$ which we already covered in subsection 2.2.3. These include access control, the ability to switch between navigation and annotation modes and the visualisation of placeholders for annotations.

We also reviewed four modern annotation systems and then listed down some of the key features and characteristics that differentiated them. These annotation systems are: i) Annotator ${ }^{1}$; ii) Factlink ${ }^{2}$; iii) Hypothes.is ${ }^{3}$; and iv) Genius ${ }^{4}$. During this process we found that some of the annotation systems had other features of interest that were not covered previously. These include the ability to respond to annotations, tagging and user participation.

The results of the aforementioned review are in Table 3.1.

\footnotetext{
${ }^{1}$ http: //annotatorjs.org

${ }^{2}$ https://factlink.com

${ }^{3}$ http://hypothes.is

${ }^{4}$ http://genius.com
} 


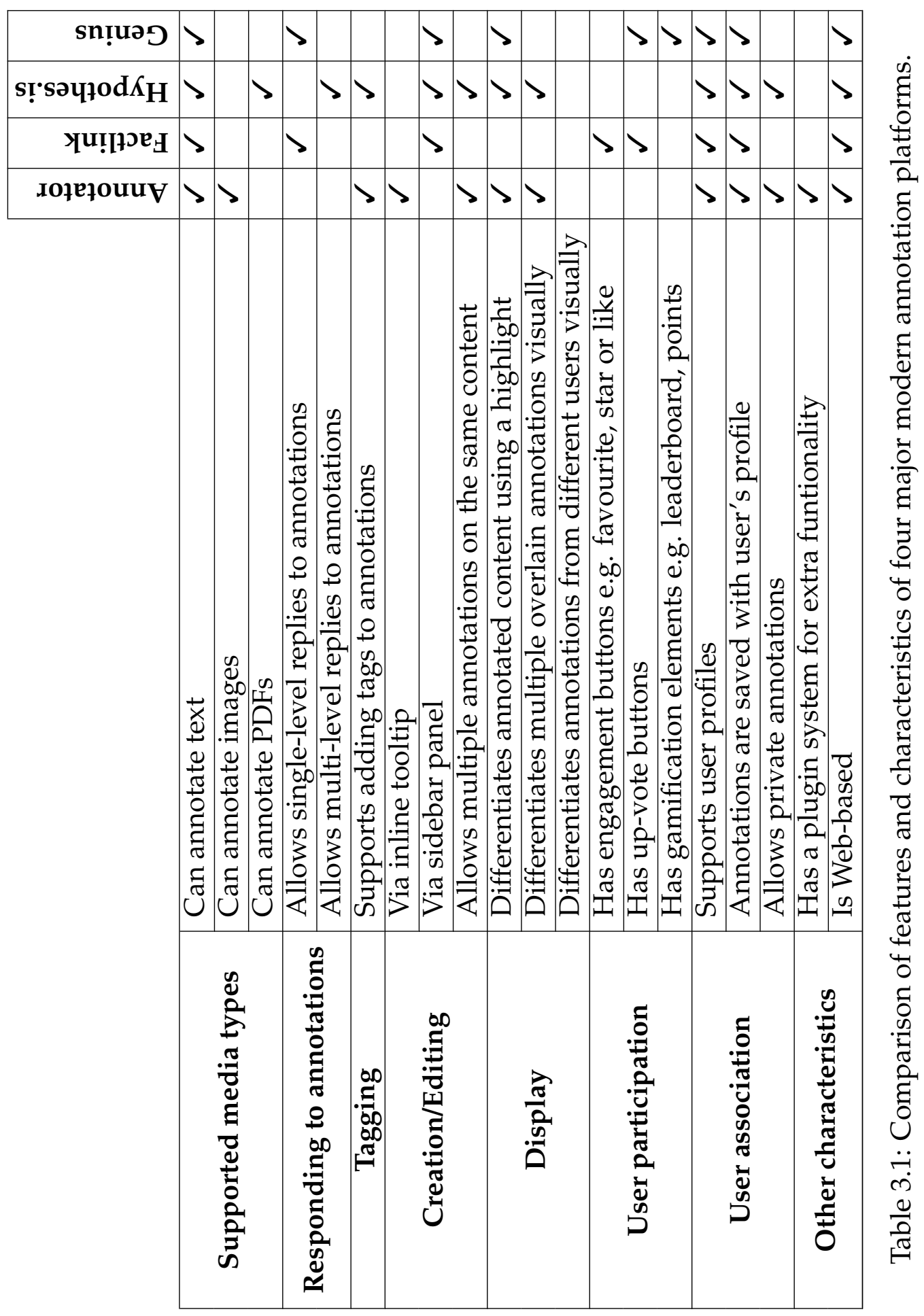

61 of 144 


\subsection{Confirming Requirements}

Each annotation system had a varying feature set that catered to its main objective. For example, Factlink focused on the validation of facts and therefore had tools that allowed the user to vouch for their credibility via the annotation system. Regardless of the objective, there were attributes that were synonymous to each annotation system albeit with some differences in their user interfaces.

We therefore listed the requirements of the system we intend to develop for our experiment which were a subset of the established requirements discussed in section 3.2. These checklists underpinned our selection criteria of what system to use and the requirements therein were deemed achievable based on the aforementioned review. We split these requirements into three categories [88]:

- Functional - Capture what the intended system should do. See Table 3.2.

- Data - Outline the type, volatility, size/amount, persistence, accuracy and even value of the required data. See 3.3.

- Technical - Specify what technologies the system is required to run on, what it needs to be compatible with or what technological limitations might be relevant. See 3.4.

From a functional point of view, Factlink, Hypothes.is and Genius were unsuitable for our experiment because they did not support annotation of images. This was a requirement because we intended to support annotation of images on our digital heritage archive of choice. It is important to note that Annotator does not support images by default, however, it is possible to use an external plugin to add the image annotation feature. See Table 3.2.

Another functional limitation of Factlink and Genius was that they did not visually distinguish annotations. Annotator and Hypothes.is used varying shades of the highlight colour while the rest used a single shade of the hightlight colour. This would have made it difficult to differentiate multiple overlain annotations.

All annotation systems fulfilled the data requirements as shown in Table 3.3. However, only Annotator fulfilled all the technical requirements as shown in Table 3.4. Extensibility enabled us to add features that we would need in our experiment. For example, Annotator's extensibility enabled us to add address a missing functional requirement i.e. we were able to build a custom up-vote feature. The up-vote feature was crucial because it was one of the user metrics that we planned to use to calculate gamification scores. 


\begin{tabular}{|c|c|c|c|c|}
\hline Functional Requirements & 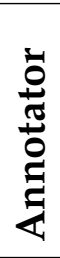 & 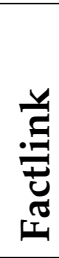 & 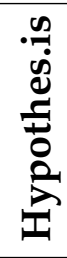 & 苞 \\
\hline User can annotate a block of text & $\checkmark$ & $\checkmark$ & $\checkmark$ & $\checkmark$ \\
\hline User can annotate a section of an image & $\checkmark$ & & & \\
\hline User can edit of delete an annotation & $\checkmark$ & $\checkmark$ & $\checkmark$ & $\checkmark$ \\
\hline User can view previously created annotations & $\checkmark$ & $\sqrt{ }$ & $\checkmark$ & $\checkmark$ \\
\hline User can favourite or up-vote an annotation & & $\checkmark$ & & $\checkmark$ \\
\hline User can visually distinguish annotations & $\checkmark$ & & $\checkmark$ & \\
\hline User's annotations are associated with their profile & $\checkmark$ & $\checkmark$ & $\checkmark$ & $\checkmark$ \\
\hline User can view previous annotation activity & $\checkmark$ & $\sqrt{ }$ & $\checkmark$ & $\checkmark$ \\
\hline
\end{tabular}

Table 3.2: Checklist of the fulfilment of functional requirements vis-a-vis selected annotation systems.

\begin{tabular}{|c|c|c|c|c|}
\hline Data Requirements & 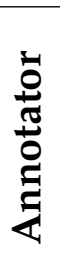 & 弑 & 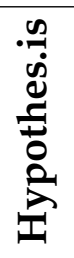 & 莺 \\
\hline Can ascribe an annotation to an identifiable user & $\sqrt{ }$ & $\checkmark$ & $\checkmark$ & $\checkmark$ \\
\hline Can persist created annotations for future retrieval & $\sqrt{ }$ & $\sqrt{ }$ & $\checkmark$ & $\sqrt{ }$ \\
\hline
\end{tabular}

Table 3.3: Checklist of the fulfilment of data requirements vis-a-vis selected annotation systems.

\begin{tabular}{|c|c|c|c|c|}
\hline Technical Requirements & 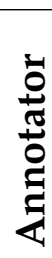 & $\underset{ت}{\mathscr{Z}}$ & 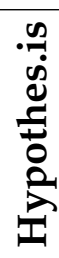 & 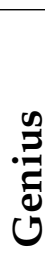 \\
\hline Can be integrated with an external website & $\sqrt{ }$ & $\sqrt{ }$ & $\sqrt{ }$ & \\
\hline Is Web-based & $\checkmark$ & $\checkmark$ & $\checkmark$ & $\checkmark$ \\
\hline Is extensible (ability to add extra functionality) & $\sqrt{ }$ & & & \\
\hline
\end{tabular}

Table 3.4: Checklist of the fulfilment of technical requirements vis-a-vis selected annotation systems. 
We came to the conclusion that Annotator's feature set was exactly what we needed for our research experiment because we would be able to:

1. Support creating, editing and deleting of annotations.

2. Annotate both text and images.

3. Save users' annotations along with their user data for future viewing.

4. Integrate it with an external website.

5. Extend it via custom plugins so that we could add functionality that is required for our study but not available in Annotator by default.

\subsection{The Digital Bleek and Lloyd Collection}

The digital heritage archive of choice for this study was the Digital Bleek and Lloyd Collection which is an archive of Khoisan heritage formed from the digitised records of Lucy Lloyd and Wilhelm Bleek's notebooks [3].

These records consist of scans of the pages of their notebooks as well as various drawings made by the Khoisan. The collection also includes a searchable word index that is cross-referenced with notes and summaries for each of the stories listed [3]. See Figure 3.1.

\subsubsection{The Source Data}

The Digital Bleek \& Lloyd archive is implemented as a static website and therefore does not have an underlying database. The $\mathrm{XHTML}^{5}$ pages are pre-generated from $\mathrm{XML}^{6}$ source data using XSLT ${ }^{7}$. This conversion is not done client-side as some browsers do not support client-side XSLT [90]. Conversion is therefore done once and, when complete, the generated files form the collection. This collection can then be browsed like a typical webpage by clicking on hyperlinks that link up the various pages.

The XML data used in the second pass is actually the result of a first pass in pre-processing the raw data. The raw data used includes a total of 752 drawings in $\mathrm{JPEG}^{8}$ format and a human-generated Excel document containing metadata about each of the images. This metadata is easy to correlate with the actual image file due to methodical organisation of the files using unique filenames and a few high level directories [90].

\footnotetext{
${ }^{5}$ Extensible Hypertext Markup Language

${ }^{6}$ Extensible Markup Language

${ }^{7}$ Extensible Stylesheet Language Transformations

${ }^{8} \mathrm{~A}$ form of image compression format often having the .jpg or .jpeg file extension. The abbreviation stands for 'Joint Photographic Experts Group'.
} 


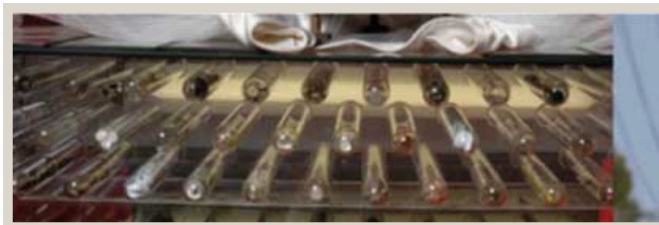

THE DIGITAL BLEEK AND LLOYD

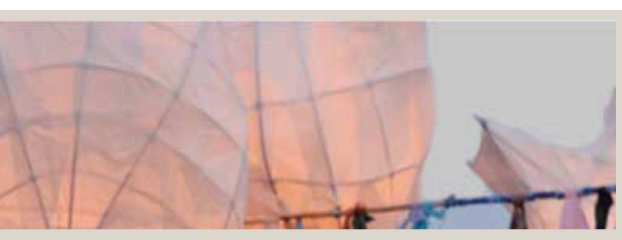

HOME

This digital publication is part of a Llarec project to digitise, research and publish the Bleek and Lloyd Archive. The Digital Bleek and Lloyd includes scans of every page of the 110 Lucy Lloyd |xam notebooks, 17 Lloyd (mostly) !kun notebooks and 28 Wilhelm Bleek |xam notebooks. It also includes Jemima Bleek's solitary Korana and !kun notebook and four Lloyd Korana notebooks in the Maingard collection of the Library at the University of South Africa, as well as Dorothea Bleek's 32 notebooks. All the drawings and watercolours made by |han $\neq k a s s ' 0$, Dia!kwain, Tamme, luma, !nanni and Da are also in the digital collection. The digital archive includes a 280000 -word searchable index, cross-referenced and including notes and summaries for each of the stories listed. Notes in italics are direct quotes from the reports of Bleek and Lloyd in which they detailed the progress of their research.

Llarec (the Lucy Lloyd Archive, Resource and Exhibition Centre) is part of the Centre for Curating the Archive, a University of Cape Town research centre directed by Pippa Skotnes and located at the Michaelis School of Fine Art. The initial "Digital Bleek and Lloyd" accompanied the publication "Claim to the Country: the Archive of Wilhelm Bleek and Lucy Lloyd" by Pippa Skotnes (2007), published by Jacana Media and Ohio University Press. Subsequently Jemima Bleek's and Dorothea Bleek's notebooks have been added, as well as the Digital Stow, featuring the rock art copies of George Stow. The search index and summaries have also been extended and currently the Bleek and Lloyd dictionaries are being digitised. Please refer to the CCA website at http://www.cca.uct.ac.za for updates.

The project has been made possible by funding provided by the Andrew W. Mellon Foundation and De Beers; and is the result of the cooperation of the four curating institutions: University of Cape Town, Unisa, Iziko South African Museum and The National Library of South Africa.

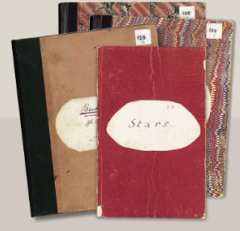

Books

Cover to cover I Contributor Story I Category | Keyword

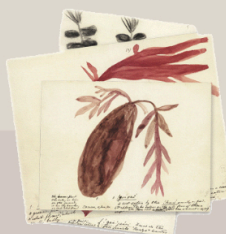

Drawings/Watercolours

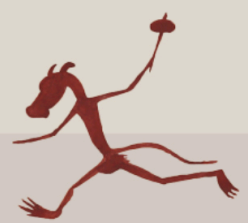

Digital George Stow Images

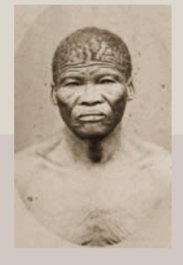

Contributors

Figure 3.1: The Digital Bleek \& Lloyd Collection.

\subsubsection{Recreating the Archive for Testing}

The approach above used by Suleman proved that XML, XSLT and XHTML can be used to generate a usable and useful static and portable digital library [90].

Taking advantage of the interoperability, it was easy to manipulate the source XML data to recreate a prototype for testing feasibility of the design choices. The tools of choice were Ruby ${ }^{9}$, Nokogiri ${ }^{10}$ and Jekyll ${ }^{11}$.

Ruby was singled out as the programming language of choice, not because it offered superior tools, but simply because the researcher's proficiency offered time-saving advantages. This translated into rapid regeneration of the collection from XML in a way that would be edited easily to test out various functionality

\footnotetext{
${ }^{9}$ https://www.ruby-lang.org

${ }^{10}$ http://www.nokogiri.org

${ }^{11}$ http://jekyllrb.com
} 
expected to be in the prototype.

Nokogiri is a Ruby HTML, XML, SAX ${ }^{12}$, and Reader parser library. Internally it depends on libxml2 and libxslt and therefore seems to be a Ruby wrapper to XML and XSLT system libraries. Among Nokogiri's many features is the ability to search documents via XPath ${ }^{13}$ or $\mathrm{CSS}^{14}$ selectors. It was used to convert the XML source to a format that can be integrated into Jekyll.

Jekyll is a static site generator that transforms plain text into static websites. Coupled with Nokogiri to parse the XML source data, a subset of the digital Bleek and Lloyd collection was quickly and easily recreated. Some of Jekyll's features offered advantages for rapid prototyping, such as the built-in development server with the ability to watch for changes in local files. This feature enables one to view the changes to the files in realtime by recreating the entire collection from the extracted XML source into static HTML files automatically. By defining templates for the pages with similar structure, Jekyll is able to generate all the relevant pages from the source data.

\subsection{System Overview}

The Digital Bleek \& Lloyd Collection was enhanced with annotations (both images and text) and gamification. This involved adding custom JavaScript to all the pages of the digital heritage archive.

The JavaScript was used to power the dynamic elements on the pages only. Dynamic elements here refers to those elements that are built on request by JavaScript as the user interacts with the archive (as opposed to static elements that are unchanging and form the content of the archive). These elements provide functionality such as presenting gamification widgets to the user, as well as enabling them to create, view, edit and delete annotations.

The system therefore consisted of two main parts:

1. Annotation Engine - When a user creates, deletes, views or edits an annotation on the archive, those user interactions are converted into API requests that are transferred via HTTP to the annotation engine. This engine exists on a different server than the archive and so it can even be used to annotate other digital heritage archives at the same time. All it would take in such a case would be to add the custom JavaScript to the other target digital heritage archives in the same way.

\footnotetext{
${ }^{12}$ Simple API for XML

${ }^{13}$ XML Path Language, a query language for selecting nodes from an XML document.

${ }^{14}$ Cascading Style Sheets
} 
In addition to storage, another key functionality of the annotation engine was handling of permissions. Each annotation was attached to a single user and the owner of an annotation was granted administrator rights to the annotation. This means that they were able to view, edit, delete and even grant other users permissions on the annotation (viewing and editing only).

The RESTful JSON API provided by the annotation engine is itself a Rails application serving as an interface to a PostgreSQL database. See Section 3.6 for details on the specifics of the API endpoints.

2. Enhanced Archive - JavaScript was used on the digital heritage archive to create UI widgets to support user interaction. Custom JavaScript had to be written to merge text annotation support, image annotation support, permissions support and serialising the annotations for interaction with the annotator store API endpoints.

Despite the fact that the two core components existed on different servers and that the digital heritage archive was a static site, it was still possible to authenticate users across different domains by making use of browser cookies. As long as the user's session had valid authentication cookies (for the annotation engine domain), any AJAX request sent via JavaScript from the digital heritage archive would be deemed authenticated.

The overall design of the system is illustrated in Figure 3.2.

This architecture was preferred because it was the least destructive approach to modifying the digital heritage archive, thus simplifying the integration process. In fact, the major part of the work required was in building the annotation engine itself.

\subsubsection{Interaction With Text Annotations}

\section{Discovering The Text Annotation Feature}

The text annotation feature was made available to the user using a blue pen tooltip widget that appeared only when a user highlighted any portion of text. This tooltip was intended as a hint to the user to take further action. See Figure 3.3.

\section{Creating Text Annotations}

To create a text annotation, the user selects a portion of text and clicks on the blue pen tooltip, after which they will be presented with an annotation widget. The widget contains a text box where the user types out the content of the annotation. 


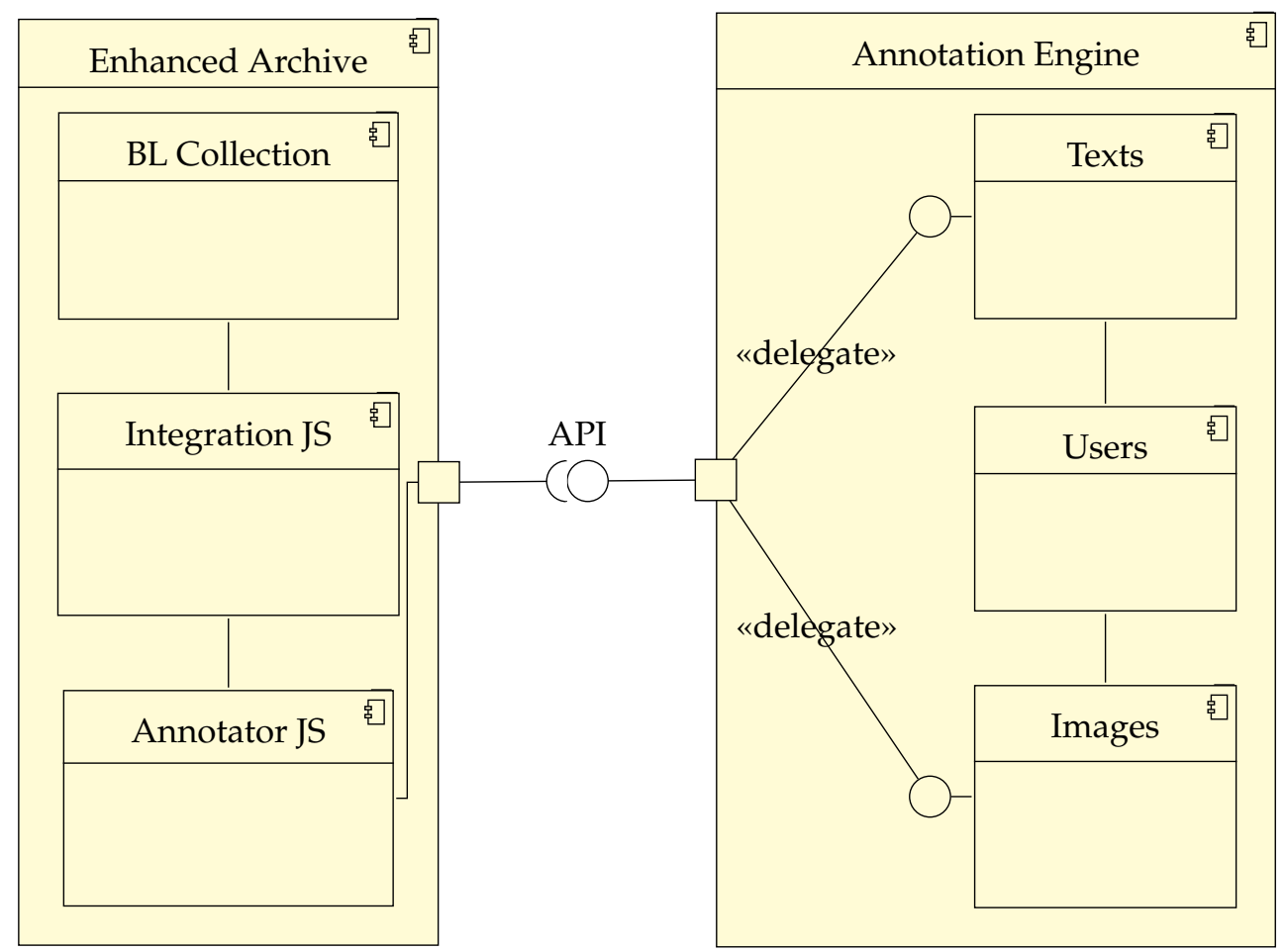

Figure 3.2: Component digram illustrating how every element of the system ties in together.

The widget also has permission controls that allow the user to specify if the annotation should be publicly viewable and if other users should be able to edit its contents. See Figure 3.4 .

\section{Viewing Text Annotations}

Once an annotation is created, it's marked by a yellow highlight. To view the annotations made, the user hovers the mouse over the yellow highlight to reveal the view widget. The view widget shows the content of the annotations, edit/delete buttons, the username of the annotator and an up-vote information section. The up-vote section has a checkbox for users to up-vote the annotation and also a count of the total number of up-votes that the annotation has received. See Figure 3.5.

In the case that multiple users annotate the same portion or overlapping portions of text, this overlap is represented using a deeper shade of yellow. The annotations are also threaded in the view widget to represent them as if they were part of one conversation. See Figure 3.6. 


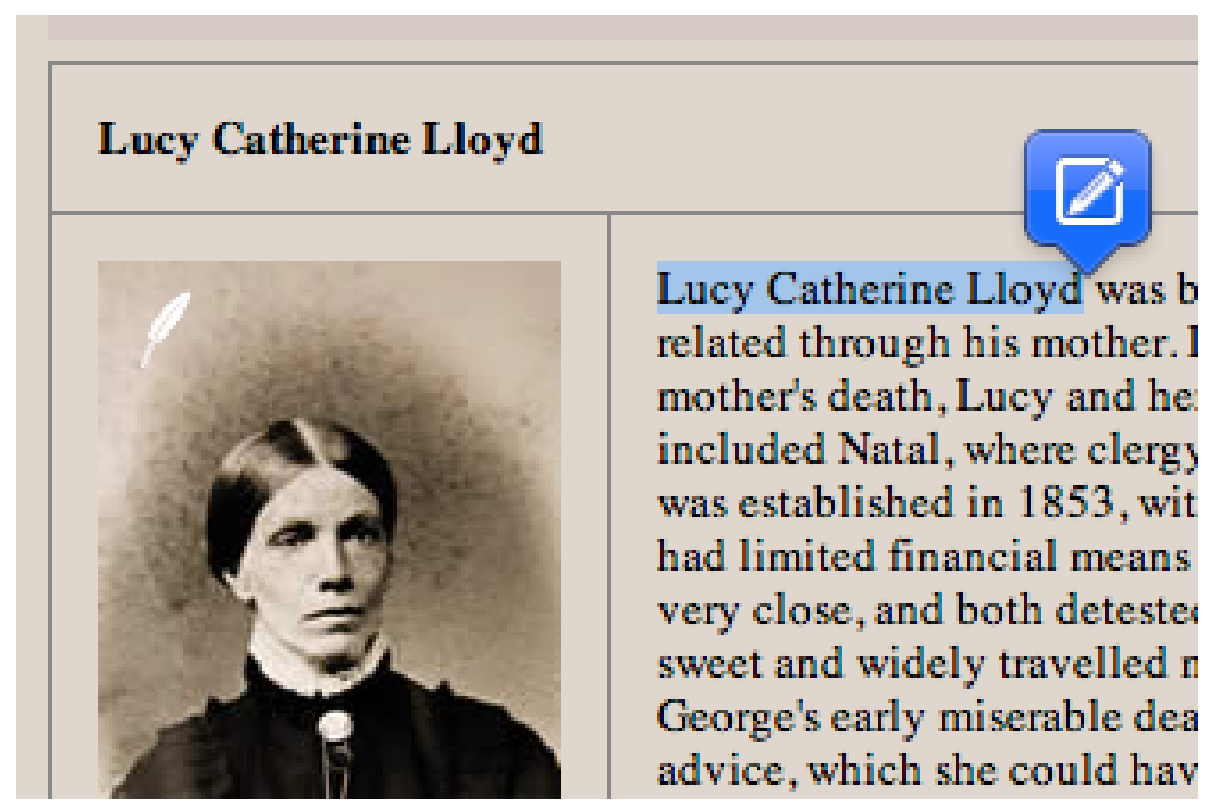

Figure 3.3: Blue pen tooltip widget (top right) used to aid in discovery of text annotation feature.

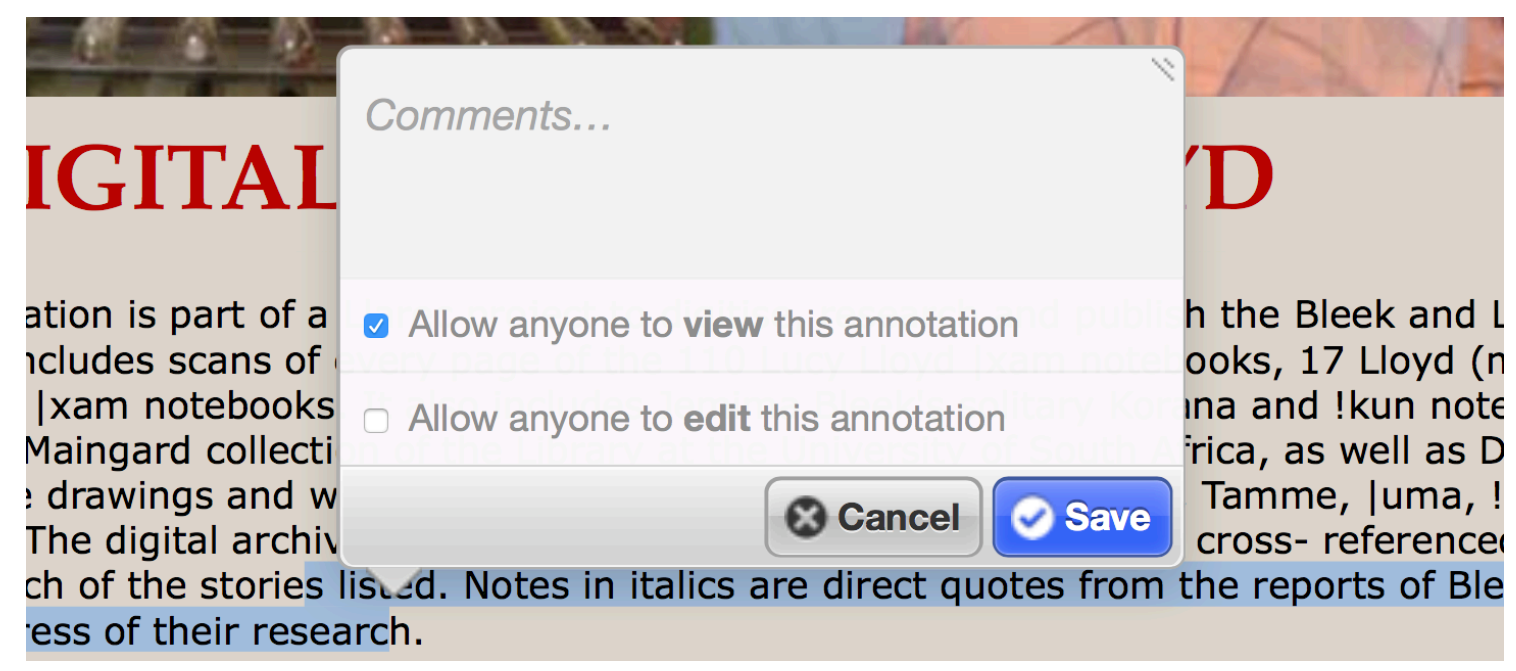

Figure 3.4: Annotation widget used when creating and editing a text annotation (with permission options below the text box).

\section{Editing Text Annotations}

The user is also able to edit an annotation after creating it. In the view widget shown in Figure 3.5, the edit button is on the top right of the widget and is symbolised by a pencil icon. 
roject to digitise, I

ge of the 110 Luc This is an example of the content of

ive. The Digital

includes Jemima $\mathrm{E}$ an annotation

$\geq$ Library at the Un

urs made by Ihan

es a 280000 -wori

jobkingori

otes in italics are

๑ Upvote! un notebooks and I four Lloyd Korana 3leek's 32

I $\mathrm{Da}$ are also in the luding notes and

1 Vote oyd in which they

nd Exhibition Centre) is part of the Centre for Curating the Archive, a University of pa Skotnes and located at the Michaelis School of Fine Art. The initial "Digital Bleek laim to the Country: the Archive of Wilhelm Bleek and Lucy Lloyd" by Pippa Skotnes

Figure 3.5: Annotation widget used when viewing an annotation (edit and delete buttons are on the top right).

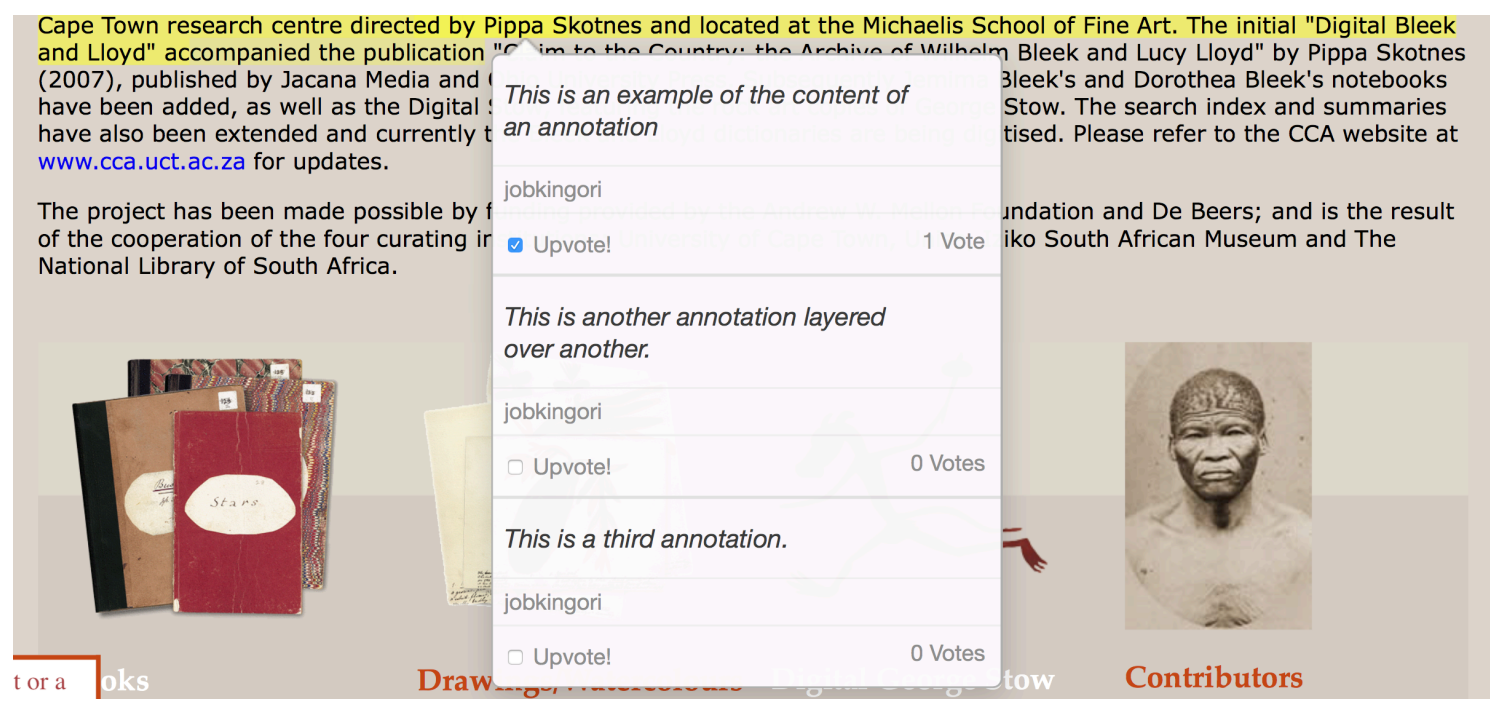

Figure 3.6: Annotation view widget showing threaded annotations (multiple annotations on same portion of text).

Once the user clicks the icon, they are presented with a similar widget to the one used when creating an annotation (see Figure 3.4). The only difference is that the values are populated with previously submitted values that were persisted on saving the annotation.

\section{Deleting Text Annotations}

To delete an annotation, the user clicks on the icon marked by an ' $X$ ' that is next to the edit button. This will send a request to the server to delete the record 
associated with that annotation and also removes the yellow highlight marking the portion of text that was annotated.

\subsubsection{Interaction With Image Annotations}

\section{Discovering The Image Annotation Feature}

The availability of the image annotation feature was hinted to the user by a white feather widget on mouse hover. The white feather appeared on the top left of any image that was available for image annotation. This widget was intended as a hint to the user to take further action. See Figure 3.7.

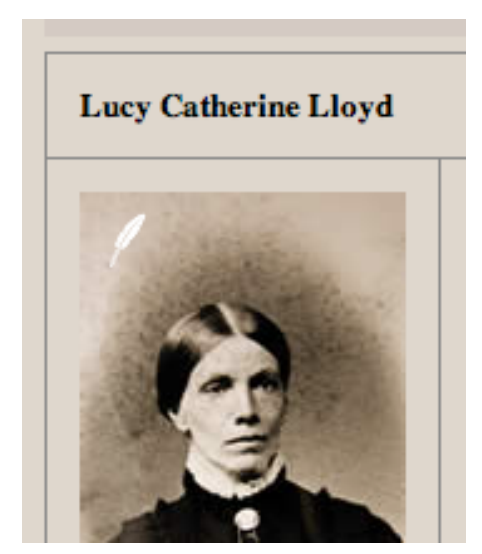

Figure 3.7: White feather widget (top left of photo) used to aid in discovery of the image annotation feature.

\section{Creating Image Annotations}

Annotating an image follows a similar process as the one used when creating a text annotation. The only difference is that, instead of selecting a portion of text, the user selects the portion of an image. The section of the image to be annotated is then represented as a bounding box. See Figure 3.8.

\section{Viewing Image Annotations}

Once an image annotation has been created, the section that has been annotated has an overlay of the aforementioned bounding box. On mouse hover, a view widget similar to the one used to view text annotations is displayed. See Figure 3.9 . 


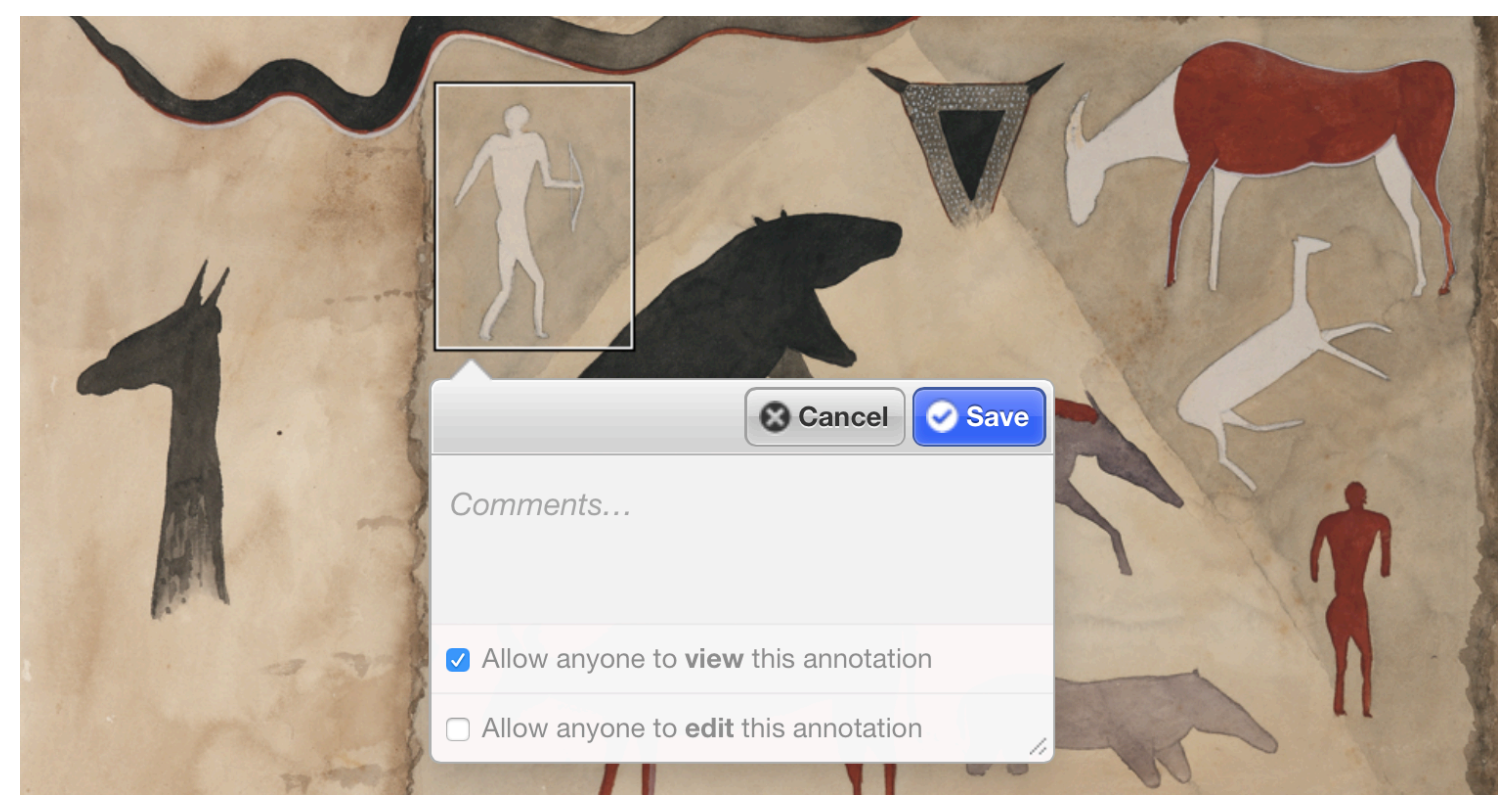

Figure 3.8: Annotation widget used when creating and editing an image annotation (with permission options below the text box).

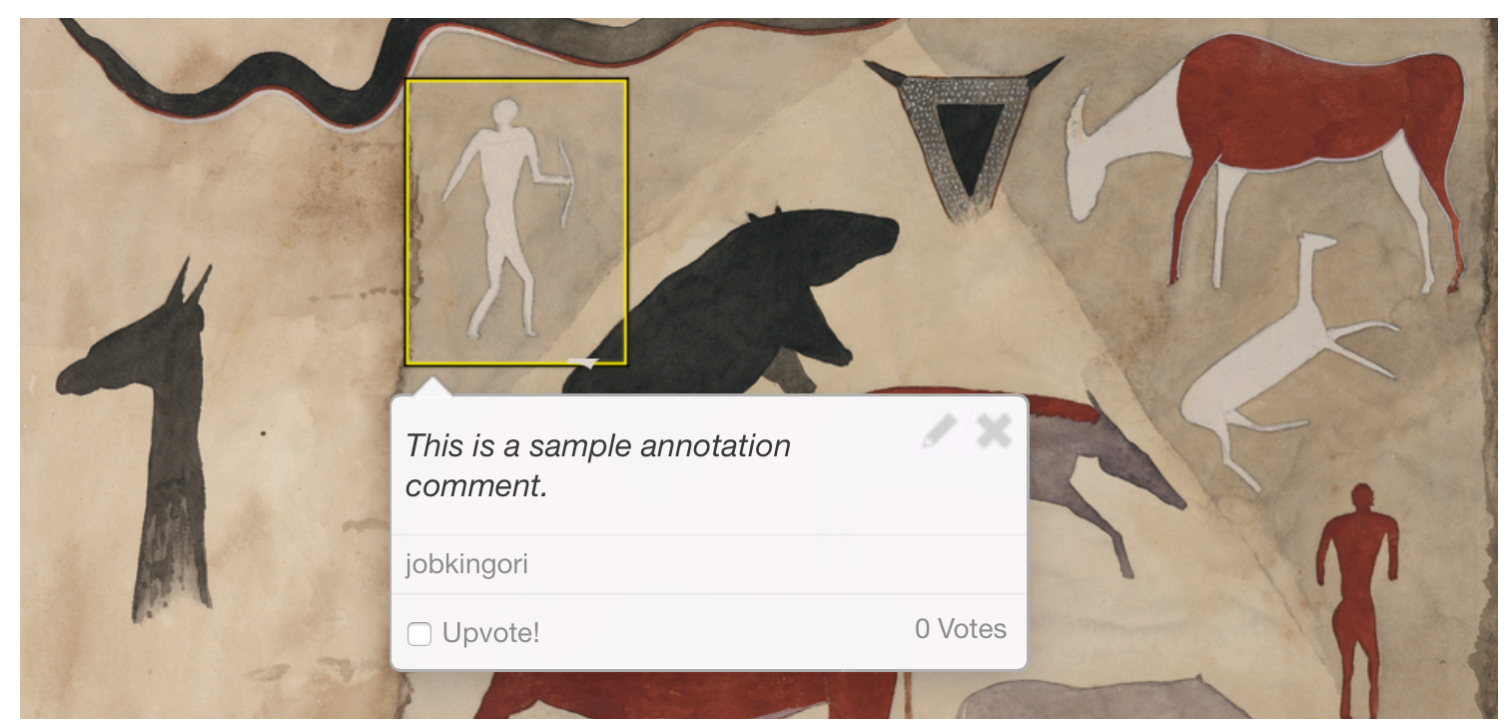

Figure 3.9: Annotation widget used when viewing an image annotation (edit and delete buttons are on the top right).

\section{Editing And Deleting Image Annotations}

The process to edit and delete image annotations is exactly similar to the processes used to edit and delete text annotations. This is because the only difference 
between making image annotations when compared to making text annotations is in how you select the section being annotated.

\subsubsection{Gamification Elements}

\section{Score Leaderboard and Up-vote Counts}

A leaderboard was featured on the left of the digital heritage archive showing a list of usernames and their respective total scores. This served as a ranking system for the users. See Figure 3.10.

In addition to the leaderboard, the total count of points derived from up-votes was also displayed alongside the annotator's name in the view widget of both text and image annotations. See Figure 3.5 and Figure 3.9 respectively.

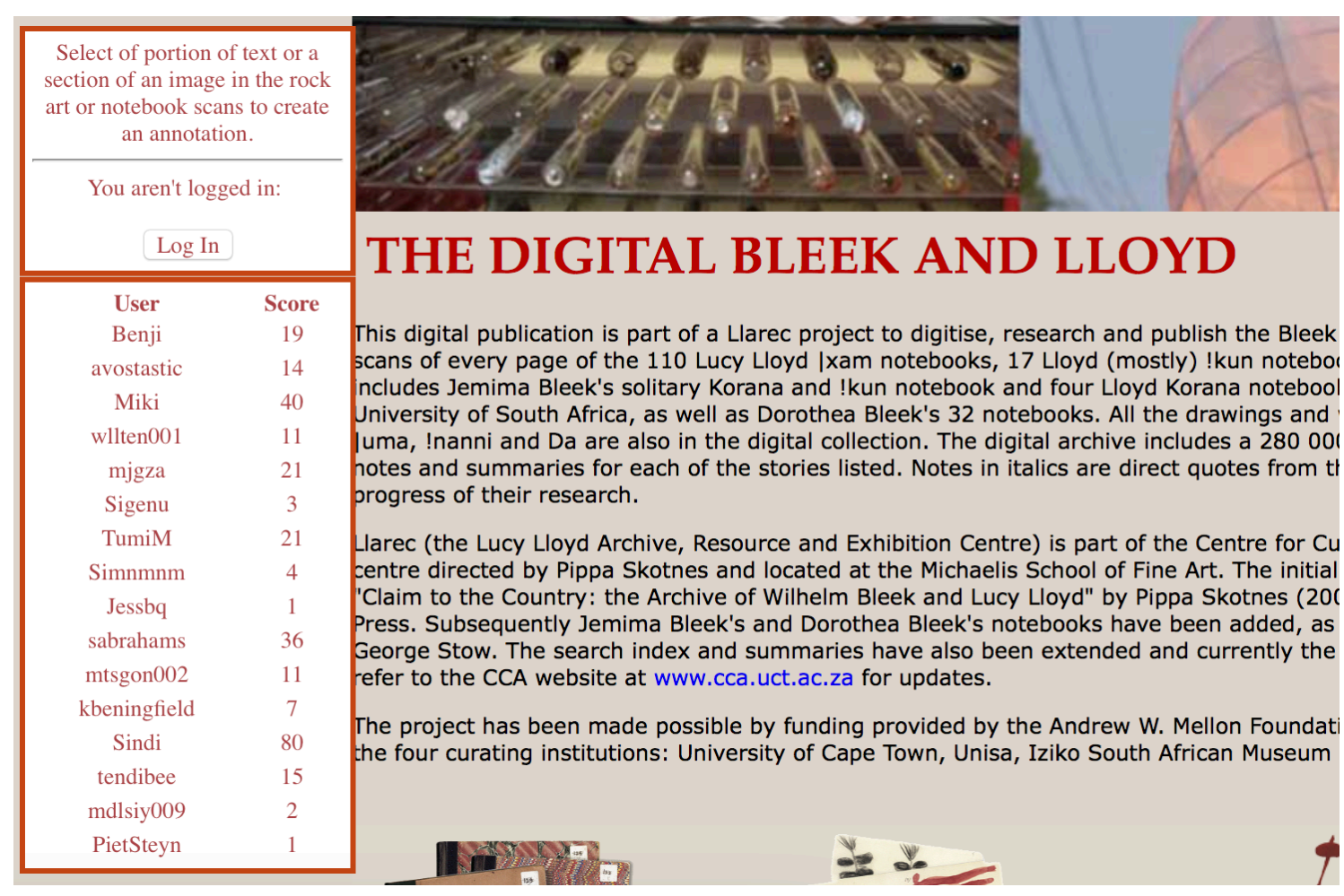

Figure 3.10: Leaderboard showing the usernames of different users and their respective scores which were derived from totals of annotations and up-votes.

\section{Calculating User Scores}

A scoring system was created to serve as a measure of participation in the core activity and the quality of annotations. With each annotation and up-vote ascribed 
a value of one point, the scoring formula for both text and image annotation activities was based on the number of annotations a user made and the sum of up-votes on all their annotations. See Equations 3.1 and 3.2 respectively.

$$
\begin{aligned}
\text { Text score } & =\text { No. of text annotations }+ \text { Sum of up-votes } \\
\text { Image score } & =\text { No. of image annotations }+ \text { Sum of up-votes } \\
\text { Total score } & =\text { Text score }+ \text { Image score }
\end{aligned}
$$

The total score was then calculated by summing up all the points from image and text annotations. See Equation 3.3.

\section{Generating The Leaderboard}

The leaderboard was generated dynamically on the user's browser using clientside JavaScript. However, the calculations of the user's score was done on the server and not on the client. Therefore, the API response was a JSON representation of the results of all the users who had created annotations.

The results used to populate the leaderboard were obtained via the stats API endpoint on the annotation engine mentioned in Section 3.6.

\subsection{Building The Annotation Engine}

To support the annotation system required for this study, a remote JSON ${ }^{15}$ REST $^{16}$ $\mathrm{API}^{17}$ was built ${ }^{18}$, based on the Annotator Store specification. The specification is a guide on how to define an API that the Annotator JS client-side library ${ }^{19}$ expects so as to function properly.

\subsubsection{API Endpoints (Server-side)}

The storage API is defined in terms of a URL, prefix and a number of endpoints. It attempts to follow the principles of REST, and produces JSON documents to be parsed by the Annotator JS client-side library [15].

Each of the endpoints for the storage API is expected to be accessible via a URL matching the pattern; scheme + domain + prefix + path. For example,

\footnotetext{
${ }^{15}$ JavaScript Object Notation

${ }^{16}$ Representational State Transfer

${ }^{17}$ Application Programming Interface

${ }^{18} \mathrm{https}$ ://github.com/itsmrwave/annotator_store-gem

${ }^{19}$ http://annotatorjs.org
} 
if the prefix is annotator_store and the URL (scheme + domain) of the API is http://example.com/, then we are able to construct the root, create and update endpoints as shown below:

- Root endpoint http://example.com/annotator_store

- Create endpoint http://example.com/annotator_store/annotations

- Update endpoint http://example.com/annotator_store/annotations/:id

\begin{tabular}{|l|l|l|l|}
\hline Endpoint & Method & Path & HTTP Response \\
\hline Root & GET & / & 200 OK \\
Create & POST & /annotations & 201 CREATED \\
Read & GET & /annotations/:id & 200 OK \\
Update & PUT/PATCH & /annotations/:id & 200 OK \\
Delete & DELETE & /annotations/:id & 204 NO CONTENT \\
Search & GET & /search & 200 OK \\
Stats & GET & /stats & 200 OK \\
\hline
\end{tabular}

Table 3.5: Annotator Store API endpoints where :id should be replaced with the annotation's actual id.

While the specification defines 7 endpoints (root, index, create, read, update, delete and an optional search endpoint) [15], only 6 of those were implemented for the purposes of this study. The index endpoint was excluded as it was not required for the annotation system to work with the supporting client-side library. The stats endpoint was a custom endpoint created to power a leaderboard feature. See Table 3.5 for a list of all created endpoints.

It is also expected that the Content-Type HTTP header should be set as ap$\mathrm{plication/json} \mathrm{since} \mathrm{the} \mathrm{data} \mathrm{exchange} \mathrm{format} \mathrm{is} \mathrm{JSON.}$

\section{Annotation Object Format}

An annotation is a JSON document that contains a number of fields describing the position and content of an annotation within a specified document. See Listing 3.1.

An example of the JSON representation of a text annotation is shown in Listing 3.1. A description of each attribute is given below: 


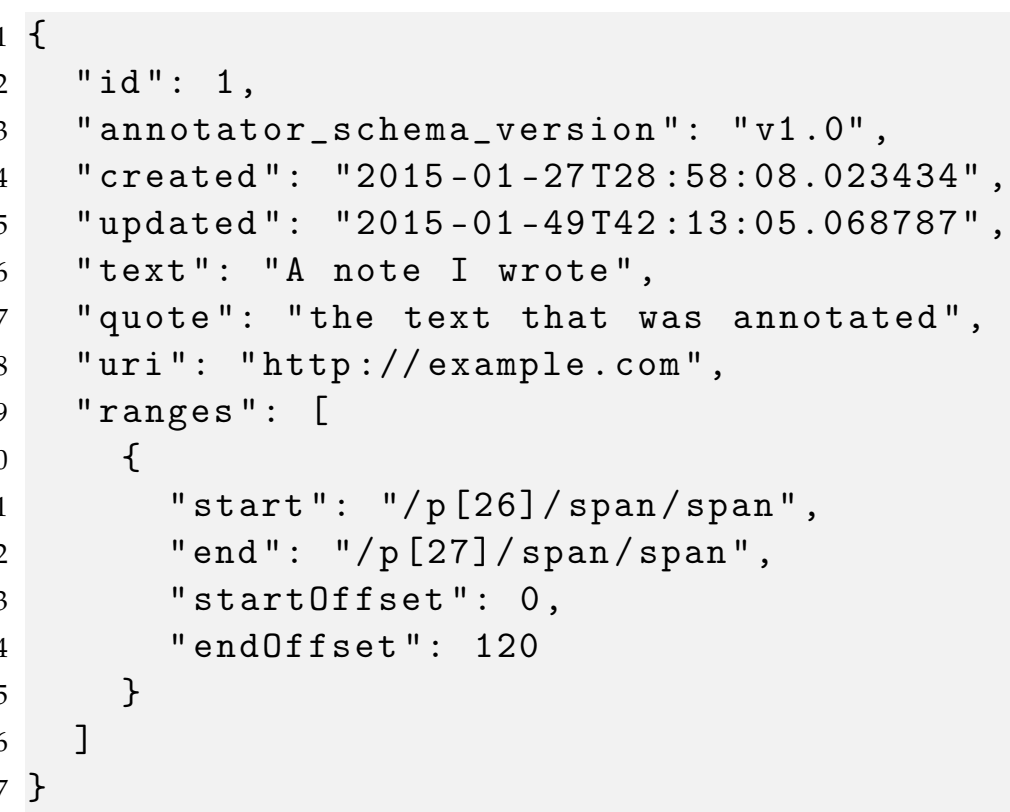

Listing 3.1: JSON annotation object format

- id - a unique identifier for the annotation (added by backend on creation). In database terms this may be regarded as the primary key of the record.

- created - date and time the annotation was created in ISO 8601 format (added by backend).

- updated - date and time the annotation was updated in ISO 8601 format (added by backend).

- text - content of the annotation (what the user typed in).

- quote - the annotated text (what the user highlighted to annotate).

- uri - URI of annotated document.

- ranges - list of descriptions defining the position of the annotation using a set of four attributes, which are:

- start - relative XPath to start element.

- end - relative XPath to end element.

- startOffset - character offset within the start element.

- endOffset - character offset within the end element. 


\section{Root Endpoint}

The root endpoint is used to provide a quick reference to other endpoints provided by the API.

It is accessible via a GET request to $\left\{A P I_{-} U R L\right.$ \}/annotator_store. The expected response is $200 \mathrm{OK}$ with the body containing a JSON object. The object contains details of the available endpoints provided by the API and other metadata. See Listing 3.2.

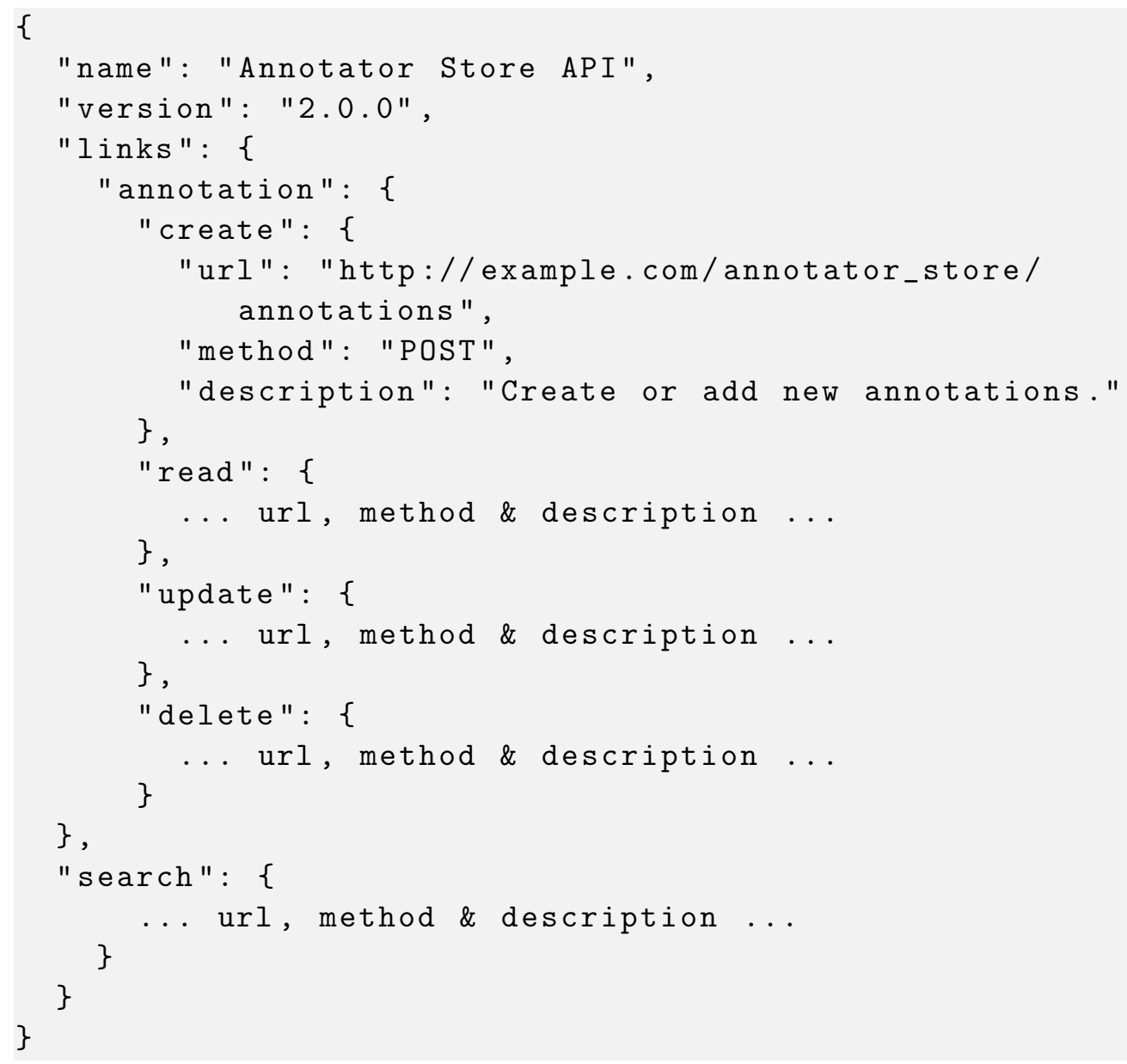

Listing 3.2: API root response structure

\section{Create Endpoint}

The create endpoint is used to persist the information passed on to it to the database, thereby saving the annotation. 
It is accessible via a POST request to \{API_URL\}/annotator_store/annotations. The expected response is 201 CREATED with the body containing the JSON annotation object representation of the created annotation. The location HTTP header of the response should also be set to the appropriate read endpoint of the annotation that has just been created. See Listing 3.3.

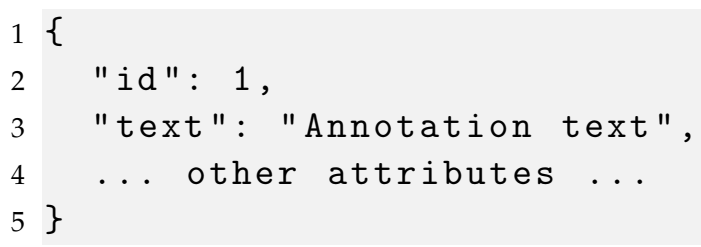

Listing 3.3: Annotation object JSON response for create, read and update endpoints

\section{Read Endpoint}

The read endpoint is used to display the details of the selected annotation. The annotation is identified using a unique id.

It is accessible via a GET request to $\{$ API_URL $\}$ /annotator_store/annotations/:id where :id should be replaced with the :id of the annotation that is being read. The expected response is $200 \mathrm{OK}$ with the body containing the JSON annotation object representation of the created annotation. See Listing 3.3.

\section{Update Endpoint}

The update endpoint is used to modify the contents of an annotation, for example, the comments by the user that accompanied the annotation when it was created.

It is accessible via a PUT or PATCH request to $\{$ API_URL $\}$ /annotator_store/annotations/:id where the :id is set just like in the read endpoint. The expected response is $200 \mathrm{OK}$ with the body containing the JSON annotation object representation of the updated annotation. The location HTTP header of the reponse should also be set to the appropriate endpoint that has just been updated. See Listing 3.3.

\section{Delete Endpoint}

The delete endpoint is used to delete the annotation from the database. 
It is accessible via a DELETE request to \{API_URL\}/annotator_store/annotations/:id where the :id is set just like in the read endpoint. The expected response is 204 NO CONTENT with an empty body.

\section{Search Endpoint}

The search endpoint is used to query the database for annotations that fit the set search criteria.

It is accessible via a GET request to \{API_URL\}/annotator_store/search. The expected response is $200 \mathrm{OK}$ with the body containing a JSON object with total and rows child elements. total is an integer representing the total number of annotations in the result set (matching the search criteria) and rows is a JSON array of each matching annotation with the structure of an annotation object. See Listing 3.4.

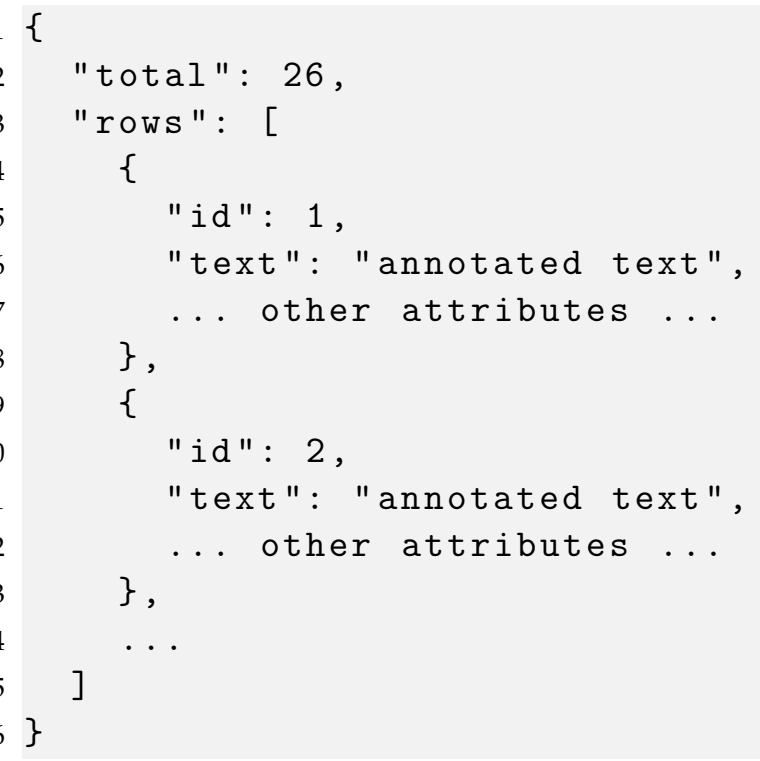

Listing 3.4: Search API response structure

The search endpoint also expects two parameters that are used to page through the results: limit and offset. When used in combination they can be used to retrieve only a portion of the rows that would be generated by the rest of the query. The limit parameter specifies the maximum number of annotations to be returned in the result set while of fset specifies the number of rows to skip.

Lastly, the endpoint also accepts the page_url parameter, which can be used to filter the annotations returned to those made on the page passed in through the page_url parameter. 


\section{Stats Endpoint}

The stats endpoint is used to query the database for the scores for the annotating users.

It is accessible via a GET request to $\left\{A P I_{-} U R L\right\} /$ annotator_store/stats. The expected response is 200 OK with the body containing a JSON array of JSON objects. Each JSON object contains a user's username, total count of annotations and sum of up-votes. See Listing 3.5.

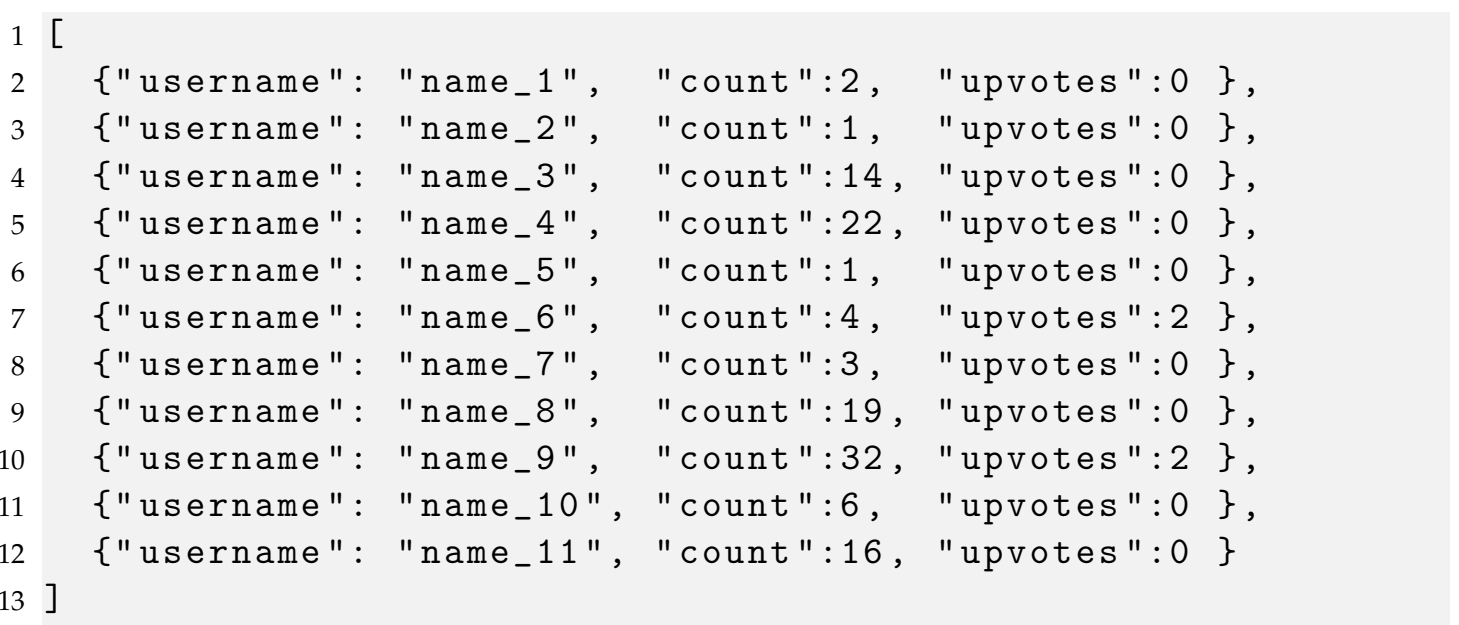

Listing 3.5: Stats API response structure

\subsubsection{Annotator Library (Client-side)}

On the user's Web browser, the Annotator JS client-side library was used to create, view, update and delete interactions to data that the Annotation Server API endpoints can consume. For more information about Annotator JS, see subSection 2.2.2.

\section{Supporting Text Annotation}

Without customisation, the Annotator JS client-side library only supports text annotations. As the user interacts with the Web page by clicking, dragging and typing during annotation, the plugin is able to record the text, quote, URI and range data. This data is then serialised into a JSON string for consumption by the API endpoint for persistence (at least in the case of creating and updating an annotation). 


\section{Supporting Image Annotation}

Supporting image annotation was more complex as the Annotator JS client-side library is not designed to support images. It is also important to note that the Annotator Store specification does not define how to represent image annotations. Code had to be implemented on the server and client-side.

On the client-side, it was possible to integrate Annotator JS with Annotorious $^{20}$, which is an independent image annotation library. Annotorious is able to serialize image annotation interactions by the user into a JSON format that can be consumed by an API. See Listing 3.6.

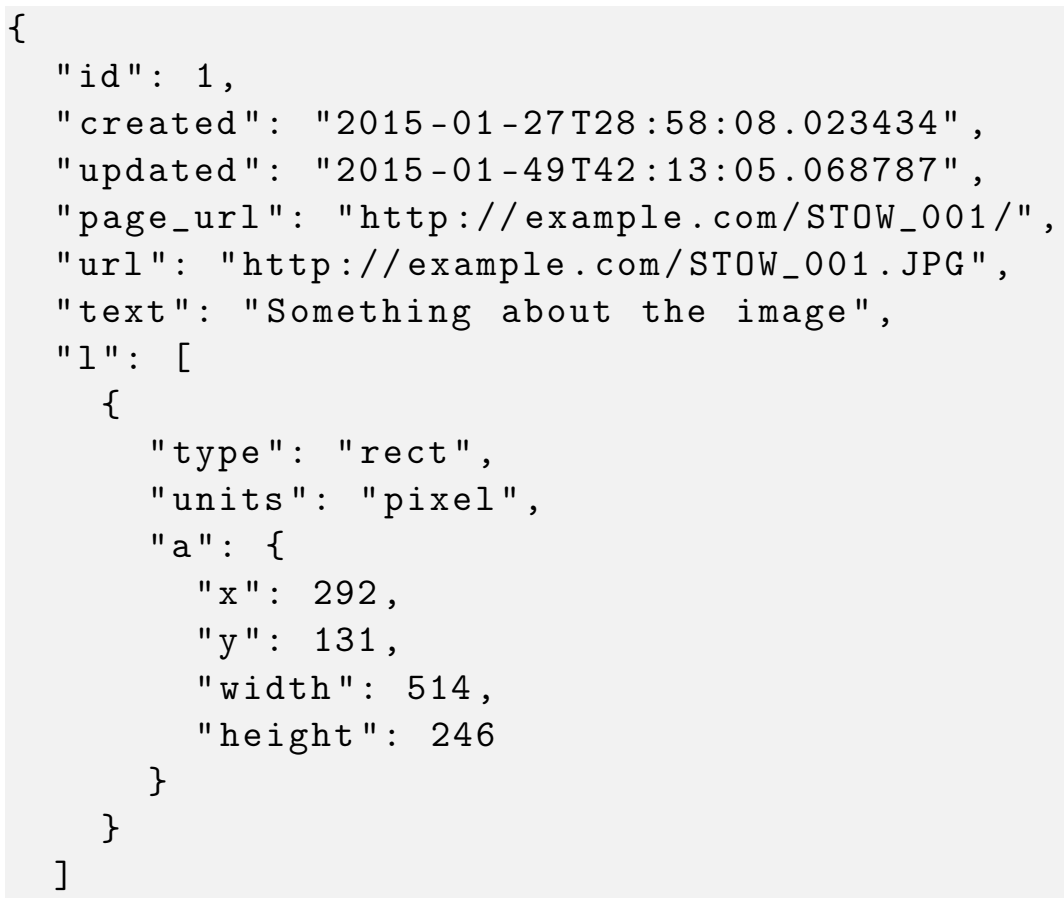

Listing 3.6: Image annotation JSON object structure

A description of each attribute is given below:

- id - a unique identifier for the image annotation (added by backend on creation). In database terms this may be regarded as the primary key of the record.

- created - date and time the annotation was created in ISO 8601 format (added by backend).

\footnotetext{
${ }^{20}$ http: //annotorious.github.io
} 
- updated - date and time the annotation was updated in ISO 8601 format (added by backend).

- page_url - URI of the page containing the image being annotated.

- uri - URI of the image being annotated.

- text - content of the annotation (what the user typed in).

- 1- list of descriptions defining the bounding box of the annotation within the image being annotated.

- type - shape defining the annotation area, typically a rectangle.

- units - type of the units used to define $x \& y$, typically a pixel.

- $\mathbf{x}$ - position on the $\mathrm{x}$-axis of the top left of the bounding box.

- $\mathbf{y}$ - position on the y-axis of the top left of the bounding box.

- width - the width of the rectangle/square defining the annotation area.

- height - the height of the rectangle/square defining the annotation area.

For the server-side, custom endpoints had to be added to support the image annotation JSON structure. This is because the image annotation is different in structure from its text annotation counterpart. See Table 3.6.

\begin{tabular}{|l|l|l|}
\hline Endpoint & Method & Path \\
\hline Create & POST & /annotations/image_annotations/ \\
Read & GET & /annotations/image_annotations/:id \\
Update & PUT/PATCH & /annotations/image_annotations/:id \\
Delete & DELETE & /annotations/image_annotations/:id \\
Search & GET & /annotations/image_annotations/search \\
\hline
\end{tabular}

Table 3.6: Image Annotation API endpoints

\section{Adding Support For User Permissions}

An annotation object (both text and image annotations) can also store permission details as shown in Listing 3.7. The user attribute contains the username of the user to which the annotation belongs and the permissions attribute assigns permissions to the current user as well as other users. These permissions detail who is able to read, update, delete and change permissions of the annotation. 


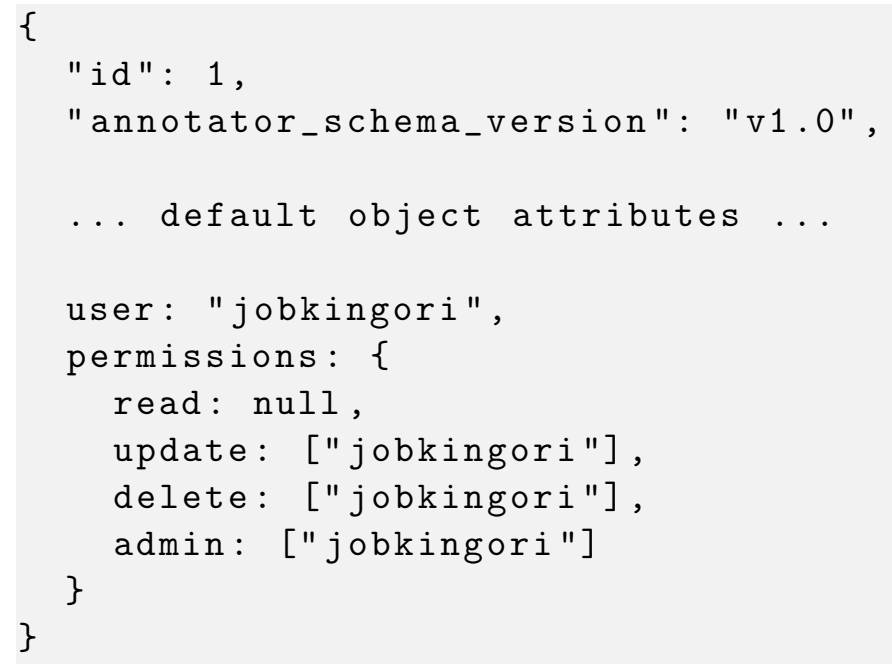

Listing 3.7: JSON annotation object that passes along permission data.

In Listing 3.7 for example, the annotation is assigned to a user with the username 'jobkingor $i$ ' who is able to update, delete and change the permissions of the annotation. Assigning 'null' to any of the actions means that any other user can perform that action and so, for the previously mentioned example, all users should have been allowed to read the annotation.

To support permissions, a separate Annotator JS plugin had to be included client-side and the server must be modified to persist the extra attributes that are passed on in the JSON annotator object.

\subsection{Integrating Annotation Engine With The Digital Collection}

\section{Authenticating Users}

Some of the endpoints on the annotation server did not require authentication, for example, the annotator_store/search and annotator_store/stats endpoints. This is because the features enabled by these endpoints were used by logged in as well as logged out users. The search endpoint was used to query all the annotations made on the page currently being viewed and the stats endpoint was used to get the scores for the leaderboard.

With the annotation server and digital heritage archive existing in separate domains, the easiest way to authenticate the users was to rely on a session key that is stored on the user's browser. API calls from the client-side JavaScript that 
are made to the server will be considered authenticated if a valid session key is stored in the _AnnotatorStoreDemo_session cookie. When the user logs in, the session key is updated and associated with the profile as well as a time frame within which that session key is valid.

Even if the user is traversing the Digital Bleek \& Lloyd Collection (separate from the domain of the annotator engine), any AJAX (Asynchronous JavaScript and XML) calls to the remote APIs will carry cookies stored by that browser assigned to the domain of the remote API and therefore still be authenticated.

Due to the plugin architecture of the Annotator JS client-side library, it was also possible to extend authentication using a special token known as a JSON Web Token. Integration with the server included exposing an authenticated endpoint that would generate a JWT each time an annotation is made. This token is then passed along with each request made to the server.

\section{Supporting Cross-Origin Requests}

CORS (Cross-origin Resource Sharing) is typically a measure taken to protect resources that are online. It involves limiting access of $\mathrm{AJAX}{ }^{21}$ calls to resources that are within the same domain as the page currently being accessed [16]. When CORS restrictions are enforced, network requests to a server must include the necessary authentication credentials. The server thereafter must respond with a Access-Control-Allow-Origin header. The client will then check if the value of the header matches the domain from which the request was made.

Typically, this should suffice for simple HTTP methods such as GET, HEAD and POST. For PUT, DELETE and OPTIONS HTTP methods, the client needs to make a preflight request to the server and then make the actual request. A pre-flight request is standard practice to make sure the server is aware of the CORS ${ }^{22}$ specification before making a request.

Due to this security feature, we had to configure the annotation server to respond with the headers as shown in Listing 3.8. This is because our annotation server and the digital heritage archive were hosted on different domains. Therefore, as per the CORS requirements, any cross-origin requests would have failed without the right headers being sent back as part of the response from the annotation server.

Functions of the headers indicated in Listing 3.8 are explained below:

- Access-Control-Allow-Headers - header indicates, as part of the response to a pre-flight request, which header field names can be used during the actual request.

\footnotetext{
${ }^{21}$ Asynchronous JavaScript and XML

${ }^{22}$ Cross-origin Resource Sharing - http: //www .w3.org/TR/cors/
} 


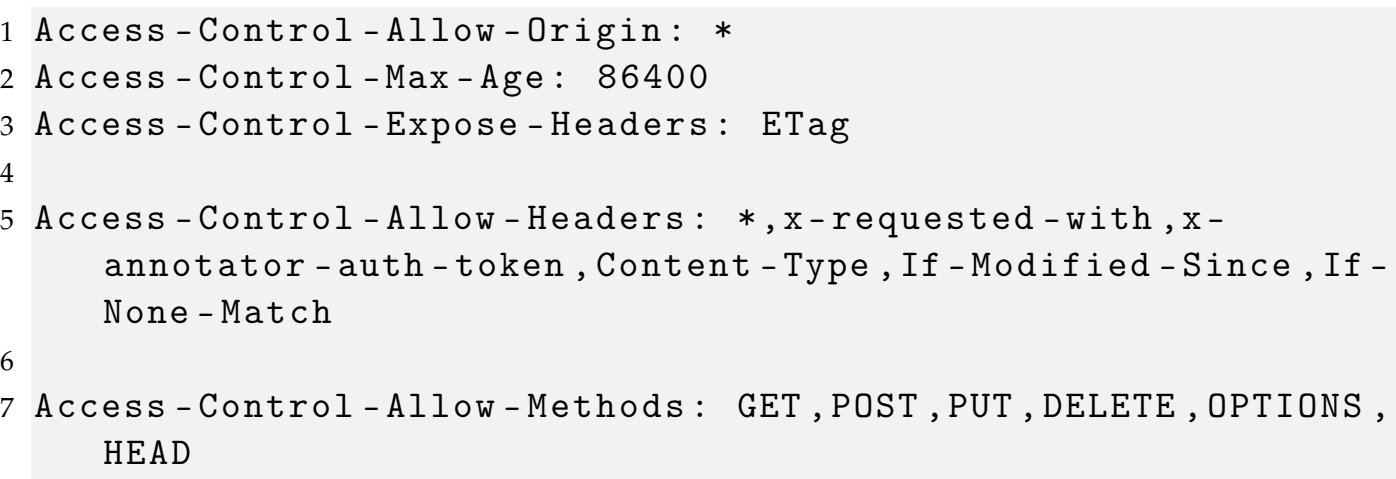

Listing 3.8: Extra headers set by the annotation server to support Cross-origin AJAX requests.

- Access-Control-Allow-Methods - header indicates the methods that can be used in the actual request.

- Access-Control-Max-Age - header indicates how long the response can be cached, so that for subsequent requests, within the specified time, no preflight request has to be made.

- Access-Control-Expose-Headers - header indicates which headers are safe to expose to the API of a CORS API specification

\subsection{Technologies and Tools Used}

The main tools and technologies used to build up the server and client interfaces are listed below:

- Amazon Web Services ${ }^{23}$ - a cloud platform provided as a service that supports multiple programming languages. This was used to host the annotation engine server that provided the API interface.

- Annotator $\mathrm{JS}^{24}$ (v1.2.5) - a JavaScript library used to add text annotation functionality on a webpage. This was used to create the widgets that enabled a user to annotate text.

\footnotetext{
${ }^{23}$ https: //aws.amazon.com

${ }^{24}$ http: //annotatorjs.org
} 
- Annotorious $\mathrm{JS}^{25}$ (v0.3) - a JavaScript library used to add image annotation functionality on a webpage. This was used to create widgets that enabled a user to annotate images.

- Foundation ${ }^{26}$ (v5.5.1.0) - a responsive front-end framework, which consists of HTML and CSS templates. This was used as a basis for styling the pages of the annotation engine such as the home, $\log$ in and sign up pages.

- jQuery ${ }^{27}$ (v1.9.0) - a JavaScript library that makes complex tasks like HTML document traversal and manipulation, event handling, animation and AJAX much simpler to do across different browsers. This was used to generate HTML elements dynamically on the client's browser as well as make AJAX calls to the annotation engine API.

- $\mathrm{JSON}^{28}$ - a lightweight data-interchange format that was used to exchange data between the server and client.

- PostgreSQL ${ }^{29}$ (v9.3.5.0) - an open-source object-relational database system. This was used to store the image/text annotations and user profile information.

- Ruby on Rails ${ }^{30}$ (v4.1.6) - an open-source Web framework written in Ruby. This was used to build the annotation engine. Rails was ideal for this because it has tools and features built in that make rapid prototyping of a Web application easy.

\subsection{Summary}

We started off the design process by reviewing four major annotation systems to understand their baseline requirements. Our review resulted in a detailed comparison, which we used to narrow down to the right tool based on our objectives. We then used the tool to develop the required system and integrated it with the Digital Bleek \& Lloyd collection.

As a result, we were able to add gamified annotations to the Digital Bleek \& Lloyd collection, which is a static site, without significant changes to the structure

\footnotetext{
${ }^{25}$ https://annotorious.github.io

${ }^{26}$ http: //foundation. zurb.com/

${ }^{27}$ http://jquery.com/

${ }^{28}$ http://json.org

${ }^{29}$ https : //www . postgresql.org/)

${ }^{30}$ http: //rubyonrails.org, (often referred to as 'Rails')
} 
of the digital heritage archive. This was achieved using a combination of clientside JavaScript and a separate backend store to persist the annotations, provide authentication and enforce permissions.

Communication between the JavaScript code on the digital heritage archive and backend store was via AJAX calls, which were made to API endpoints that were responsible for the creation, editing, viewing and deletion of annotations from the database.

The digital heritage archive could also rank users based on a scoring system that took the number of annotations and the up-votes awarded to each annotation as factors. The total points accrued by each user based on this scoring system were then viewable on a leaderboard that was featured on the digital heritage archive as the users traversed the contents of the archive.

The functionality has been extracted into a Rails engine, which has been opensourced on GitHub ${ }^{3132}$. Further details on the Rails engine can be found in Appendix A.

\footnotetext{
${ }^{31}$ https://github.com/itsmrwave/annotator_store-gem

${ }^{32}$ https://github.com/itsmrwave/annotator-store-demo
} 



\section{Chapter 4}

\section{Experimental Design}

\section{Contents}

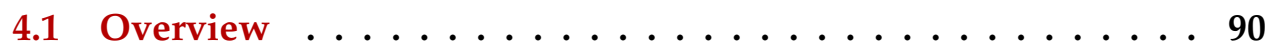

4.2 Usability Experiment $\ldots \ldots \ldots \ldots \ldots \ldots$

4.2.1 Usability Testing Prototype . . . . . . . . . . . . . . 90

4.2.2 Usability Evaluation Process . . . . . . . . . . . . . . . 91

4.2.3 Addressing Feedback from Evaluation Process . . . . . . 94

4.3 Gamification Experiment . . . . . . . . . . . . . . . 94

4.3 .1 Participation Process . . . . . . . . . . . . . . . . 95

4.3 .2 Survey Questions . . . . . . . . . . . . . . . . . . 96

4.4 Summary . . . . . . . . . . . . . . . . . 99 


\subsection{Overview}

The study revolved around two modes of an annotation framework built on data from an existing digital heritage library (The Digital Bleek and Lloyd Collection) and tested with actual users.

Section 4.2 captures the design of the usability experiment in detail while section 4.3 covers the design of the gamification experiment. The core method was centred on an experimental prototype ${ }^{1}$ and hence, for purposes of this study, an evolutionary prototyping process was preferred.

This involved building a usability testing prototype (section 4.2.1) and testing it with actual users (section 4.2.2). Feedback from that evaluation process was then used to refine it (section 4.2.3) into the final version (section 4.3), which was thereafter deployed to a live environment and used to gather data that would help answer the research questions in section 1.7.

Evolutionary prototyping involves evolving a prototype through several stages into the final product as opposed to throwaway prototyping [88]. In the latter, prototypes are used as stepping stones towards the final design and then thrown away before building the final version from scratch.

\subsection{Usability Experiment}

According to Sharp, Rogers, and Preece: prototypes are a useful aid when discussing ideas with stakeholders and an effective way to test out ideas for yourself [88]. They assist in user testing and evaluation, or to check that a certain design direction is compatible with the rest of the system development.

The scope of our usability experiment was limited to the core functionality of the annotated digital heritage archive. These include the ability to create, edit and delete text and image annotations on the Digital Bleek and Lloyd Collection using a dynamic widget interface.

\subsubsection{Usability Testing Prototype}

The primary goal of usability testing was to validate the usability of the system by placing the system in a similar controlled environment where the performance of users on pre-planned tasks was measured for later analysis [88].

Firstly, participants were selected to represent real users. It was important to make sure that the selected participants knew how to use such a system and therefore basic computer skills and the ability to use the Web were minimum

\footnotetext{
${ }^{1}$ Preliminary version based on untested ideas or techniques not yet established or finalised.
} 
requirements. Secondly, the participants were given tasks to perform on the system.

Tasks selected had to relate to the intended concerns and goals for which the designed system was created. During this exercise any feedback from the participants was noted, for example: their actions and comments. The participants were also asked questions about the system when performing the task as well as through questionnaires before the start of the exercise.

After gathering the data, both the quantitative and qualitative data were analysed together with any observations or user comments. Therefore, all these were used to document the system's usability profile such as: any issues discovered, their solutions and any new ideas that should be factored into the next iteration [44].

\subsubsection{Usability Evaluation Process}

\section{Test Environment Location And Setup}

A controlled setting was used to conduct the sessions by making use of empty and quiet rooms at the UCT Computer Science ICT4D lab. No Internet connection was required. The moderator and each participant were the only two people available at a time. The moderator in this case was the researcher.

Participants interacted with the prototype of the Digital Bleek and Lloyd collection hosted locally on the laptop while the moderator observed the participants' interactions with the archive.

The laptop in use was an Apple Macbook running Mac OS X (v10.10.1) and the preferred browser on which the system ran was Google's Chrome browser (v38.0).

\section{Sampling Criteria And Recruiting participants}

Eligibility Criteria The participants for this study had to have some basic abilities so as to be eligible. A background questionnaire was used to make sure they fit the checklist. Points to consider were:

- Must know how to use a computer

- Must know how to use the Internet

- Must know how to use a Web browser

- Must be able to read and write responses on the data collection instrument

The background questionnaire questions were tailored to investigate the participants' content consumption and content generation habits. 


\section{Survey Questions}

The U.S.E. Questionnaire (Usefulness, Satisfaction, and Ease of use) [65], based on work by Lund, was used to evaluate usability. It consists of 30 questions covering usefulness, ease of use, ease of learning and satisfaction. The questions used are listed in Tables 4.1, 4.2, 4.3 and 4.4 respectively.

\begin{tabular}{|l|l|}
\hline Code & Question \\
\hline Q1 & It helps me be more effective. \\
Q2 & It helps me be more productive. \\
Q3 & It is useful. \\
Q4 & It gives me more control over the activities in my life. \\
Q5 & It makes the things I want to accomplish easier to get done. \\
Q6 & It saves me time when I use it. \\
Q7 & It meets my needs. \\
Q8 & It does everything I would expect it to do. \\
\hline
\end{tabular}

Table 4.1: Survey questions to measure the usefulness of the usability testing prototype.

\begin{tabular}{|l|l|}
\hline Code & Question \\
\hline Q9 & It is easy to use. \\
Q10 & It is simple to use. \\
Q11 & It is user friendly. \\
Q12 & It requires the fewest steps possible to accomplish what I want to \\
& do with it. \\
Q13 & It is flexible. \\
Q14 & Using it is effortless. \\
Q15 & I can use it without written instructions. \\
Q16 & I don't notice any inconsistencies as I use it. \\
Q17 & Both occasional and regular users would like it. \\
Q18 & I can recover from mistakes quickly and easily. \\
Q19 & I can use it successfully every time. \\
\hline
\end{tabular}

Table 4.2: Survey questions to measure how easy it is to use the usability testing prototype.

\section{Process}

To begin with, the participant signed a consent form, filled in the background questionnaire and then they were taken through an introduction to the session. 


\begin{tabular}{|l|l|}
\hline Code & Question \\
\hline Q20 & I learned to use it quickly. \\
Q21 & I easily remember how to use it. \\
Q22 & It is easy to learn to use it. \\
Q23 & I quickly became skilful with it. \\
\hline
\end{tabular}

Table 4.3: Survey questions to measure how easy it is to learn how to use the usability testing prototype.

\begin{tabular}{|l|l|}
\hline Code & Question \\
\hline Q24 & I am satisfied with it. \\
Q25 & I would recommend it to a friend. \\
Q26 & It is fun to use. \\
Q27 & It works the way I want it to work. \\
Q28 & It is wonderful. \\
Q29 & I feel I need to have it. \\
Q30 & It is pleasant to use. \\
\hline
\end{tabular}

Table 4.4: Survey questions to measure the users' satisfaction while using the usability testing prototype.

During the introductory session the importance of the participant's involvement and the moderator's role was explained. They were also encouraged to 'think out loud' to encourage them to share any insights into their thought process while carrying out the required tasks [37].

The session was moderated by the researcher who would provide step by step instructions to the participant. These instructions included pre-determined tasks that the participant should carry out for the evaluation. Once these tasks were completed, the participant was then taken through a debriefing session by the moderator. The entire session took about 20 minutes per participant.

\section{User Tasks}

The participant was expected to browse the Digital Bleek \& Lloyd Collection starting from the homepage for 10 minutes or less. It was expected, but not required, that they at least make a few annotations. Making no annotations was also okay if they were unable to.

Since the participants will have been selected for different combinations of characteristics, it was also expected that different types of participants would be motivated to do different things.

Pre-defined tasks involved asking the participant to: 
1. Navigate to any page with text, select a portion of it and create and save at least two text annotations.

2. Edit any of the text annotations that they have just made.

3. Delete any of the text annotations that they have made.

4. Navigate to one of the pages in the collection with rock art images and create and save at least two image annotations.

5. Edit any of the image annotations that they have just made.

6. Delete any of the image annotations that they made.

In each of these steps the user was prodded for their insights and opinions on the functionality of the annotation system. It is also important to note that for this exercise the participant was not given specific details on how to achieve each objective so that it would be possible to see how much a typical user would be able to carry out without instruction.

\subsubsection{Addressing Feedback from Evaluation Process}

The feedback gathered from the usability study was used to improve the system and thus create the final version that would be used in the gamification experiment in section 4.3. This was limited to issues that affected the intended core functionality of the system.

One issue that was addressed affected the persistence of annotations. The system would only persist annotations as the user was viewing the page. This allowed them to create several image and text annotations on a page and have them persisted only for the duration in which they were on the page. If the user refreshed the page or navigated away from the page, any annotations created would be lost.

See sub-section 5.3.2 for all the feedback from the usability experiment.

\subsection{Gamification Experiment}

After completing the usability study, the feedback gathered was used to improve the system to create a high-fidelity prototype. This high-fidelity prototype had gamification features such as the leaderboard and the up-vote system.

Unlike the usability prototype study elaborated in section 4.2, the sampling criteria and participant recruiting process was less constrained. Invitations to participate in the gamification experiment were sent via email to the UCT student 
body. Though it was expected that the respondents would be students, there were no restrictions as to who may participate.

\subsubsection{Participation Process}

On receiving the email, the participants were expected to create an account, successfully complete user registration, browse through the Digital Bleek \& Lloyd collection, make annotations on images and text where they saw fit and then fill out a short survey. The process was as follows:

1. Visit the Annotated Digital Bleek \& Lloyd Collection at http://blannotator.kingori.co/.

2. Click on the "Sign Up" button to start the registration process, thus creating a new account.

3. A confirmation email was then sent to the email address that they specified. Clicking on the confirmation link completed the registration process.

4. Using a combination of the registered email and set password, the participant was now able to log into the archive from http://blannotator.kingori.co/.

5. Once logged in, the user was expected to click on the "Go to Bleek and Lloyd Collection" at the top of the page. Only logged in users were allowed to annotate.

6. The participant was then expected to browse the Digital Bleek and Lloyd archive while making use of the annotation tools on text or images where they saw fit.

7. At the end of their session the participant was expected to complete a survey. See section 4.3 .2 for the survey questions.

User Groups As the participants registered, they were automatically categorised into two different user groups: a gamified and an un-gamified group. The participants were only made aware of the fact that they would be split into different user groups and that each user group would get a different experience of the digital heritage archive. The criteria used to group them or what group they were in was not revealed to them.

Another difference between the two groups was in the features that they had access to. Unlike the un-gamified group, the gamified group had access to the 
leaderboard and the up-vote features. Therefore, from the point of view of the un-gamified users, all they had access to was the annotation features atop the digital heritage archive.

Remuneration Participants in both groups were given identical incentives to participate. This incentive was in the form of a monetary reward. The top 10 annotators with the highest quality (relevant and descriptive) annotations would each receive the reward tiered by rank. Likewise, the top 10 annotators with the highest number of annotations would also each receive a reward tiered by rank. The top scorer received the highest amount and each subsequent position received a little less down to the $10^{\text {th }}$ position. Only participants who successfully completed all tasks and filled in the survey qualified to claim the monetary reward.

Measurements During the experiment we recorded a number of measurements. These are: i) the number of gamified and un-gamified users; ii) the number of users who made annotations; iii) the number of up-votes that each user was awarded; and $i v$ ) the number of annotations of each type i.e. text and images .

\subsubsection{Survey Questions}

\section{Questions on the User's Background}

The purpose of these questions was to get some insight into the demographics of the participants who responded to the survey call.

The questions covered the background of the participants as well as their prior history with digital archives in general as well as digital heritage archives. The questions asked are itemised below:

- Degree - "What degree are you studying towards? Examples include: BSc., BCom., MPhil., MSc., PhD, etc."

- Major - "What are you majoring in? Examples include: Computer Science, Law, Engineering, Commerce, etc."

- Year of Arrival - "What year did you arrive at UCT? Examples include: 2012, 2013, 2014, 2015, etc."

- Gender - "What's your gender?"

- First Time In A Digital Archive - "Is this the first time browsing a digital archive/library? A digital library is a focused collection of digital objects that can 
include text, visual material, audio material, video material, stored as electronic media formats (as opposed to print, micro form, or other media), along with means for organising, storing, and retrieving the files and media contained in the library collection. Examples include: Image archives (such as Flickr), scholarly archives (such as JStor, Google Scholar), institutional repositories (OpenUCT), The Internet Archive, etc."

- Previous Digital Archives Visited - "If you answered 'no' above, please mention some of the digital archives that you have visited."

- First Time In A Digital Heritage Archive - "Is this your first time browsing one specific to heritage? - A digital heritage archive is simply an archive dedicated to the preservation of heritage related information. Examples of digital heritage archives include: Bafokeng Digital Archive, The Ulwazi Programme, Tomboucton Manuscripts Project, The Auschwitz Album, South African Rock Art Digital Archive, Google Cultural Institute, South African History Archive, etc"

- Previous Digital Heritage Archives Visited - "If you answered 'no' above, mention some of the digital HERITAGE archives you have visited."

\section{Questions on the User's Motivations}

The purpose of these questions was to find out which features of the enhanced digital heritage archive would motivate the user to carry out certain actions and to determine if gamification had an effect on those motivations.

We asked both groups of participants 7 questions that highlighted different features of the digital heritage archive. See Table 4.5 for a list of the questions.

In each question, the participant was asked if the targeted feature fed into their motivation to view, contribute to, revisit and share the archive. The participant was expected to answer each sub-question with 'Yes', 'No' or 'Uncertain'. These subquestions are itemised below:

- Viewing - "Would this feature encourage you to spend more time viewing content on the archive?"

- Contributing - "Would this feature encourage you to contribute (annotate) to the archive?"

- Revisiting - "Would this feature encourage you to revisit the archive again later?"

- Sharing - "Would this feature encourage you to share (tell others about) the archive?" 


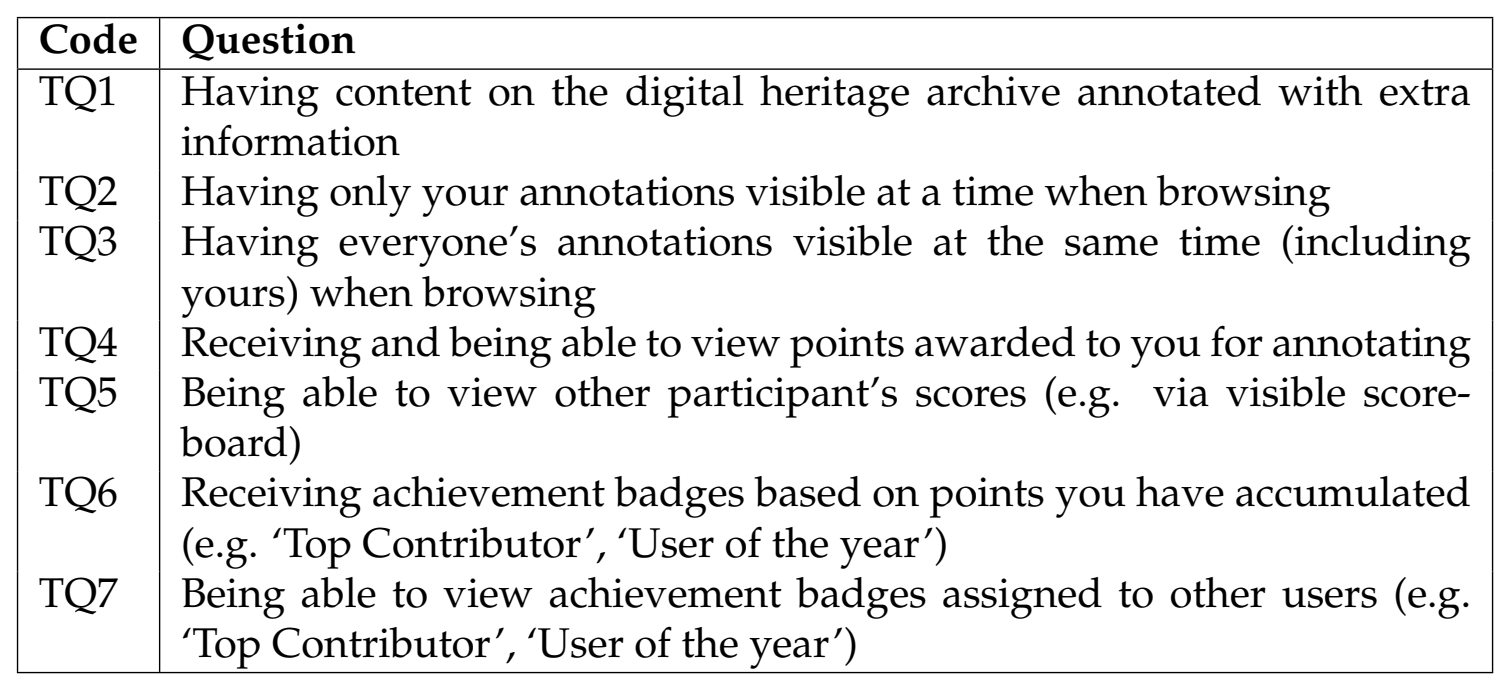

Table 4.5: Survey questions to evaluate user motivations for viewing, contributing, revisiting and sharing the archive.

Questions TQ1 to TQ3 focused on annotation features while TQ4 to TQ7 focused on gamification features. For questions TQ4 to TQ7, it is important to note that for the gamified group these questions investigated if the gamification feature did contribute to their motivations while for the non-gamified group the questions investigated if the gamification feature would contribute to their motivations.

\section{User Feedback on Usability}

We also asked both groups of participants 4 questions that were aimed at getting feedback on usability of the core aspects of the system (authentication, annotation, browsing) as well as determining its positive and negative impressions on the participants. See Table 4.6 for a list of the questions.

Our goal was to determine if gamification affected the usability of the system.

\begin{tabular}{|l|l|}
\hline Code & Question \\
\hline EOS1 & Signing up and login \\
EOS2 & Creating an image annotation \\
EOS3 & Creating a text annotation \\
EOS4 & Browsing the archive/library \\
\hline
\end{tabular}

Table 4.6: Survey questions to evaluate usability of the core functions of the system. 
Each question had 3 sub-questions. The first question investigated the ease of using the system via a 5-point Likert scale while the other two were designed as open-ended questions to encourage unguided answers. These sub-questions are:

- Ease of Use - "Did you find the following features easy to use?"

- Positive Aspects - "List any positive aspects of the system or features of the archive that you liked."

- Negative Aspects - "List any negative aspects of the system or features of the archive that you did not like."

\subsection{Summary}

We were able to design and carry out a usability experiment for the system designed in chapter 3 and also design the gamification experiment that will be used to answer the research questions outlined in section 1.7. The results of the gamification experiment are presented in chapter 5 . 



\section{Chapter 5}

\section{Results and Analysis}

\section{Contents}

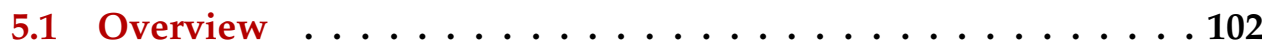

5.2 Research Questions . . . . . . . . . . . . . . 102

5.3 Usability Experiment Evaluation . . . . . . . . . . . . . 102

5.3.1 Experimental Data . . . . . . . . . . . . . . . 102

5.3.2 Usability Survey Analysis . . . . . . . . . . . . . . . . . . 104

5.4 Gamification Experiment Evaluation . . . . . . . . . . . 109

5.4.1 Experimental Data . . . . . . . . . . . . . . . . . . . 109

5.4 .2 Task Analysis . . . . . . . . . . . . . . . . . . . . 111

5.4 .3 Survey Analysis . . . . . . . . . . . . . . . . . 113

5.5 Summary . . . . . . . . . . . . . . . . . 117 


\subsection{Overview}

This chapter seeks to answer the research questions that were first enumerated in section 1.7. Section 5.2 briefly goes through the focus of these research questions. Thereafter we present and analyse the results of the usability experiment evaluation in section 5.3 and those of the gamification experiment in section 5.4. Each of these sections lays out the experimental data used and an analysis of the results.

\subsection{Research Questions}

The scope of the research questions was to test for the applicability of gamified annotations in the context of a digital heritage archive as well as their effectiveness in increasing the number and quality of annotations submitted in a digital heritage archive.

\subsection{Usability Experiment Evaluation}

\subsubsection{Experimental Data}

Recruitment Participants who are based at the UCT ${ }^{1} \mathrm{ICT}_{4} \mathrm{D}^{2}$ lab were used for this study. These were Masters and PhD students who are familiar with computers, the Web, programming and research. It was deemed appropriate to use this pool of users as they were considered to be expert evaluators of the system.

Sample Size Only 11 participants were recruited for this usability study since the purpose of this study was only to determine the usability of the system.

Participant Characteristics and Habits Out of the 11 responses used, 8 participants had MSc. degrees as their last completed degrees while the rest had BSc. degrees. 9 of the participants were men and 2 women. 4 of the participants were between the ages of 25-29 years, 5 between 30-35 years and the 36-40 and 41-45 year bands each had 1 participant.

Tables 5.1 and 5.2 show participant habits, where the frequency columns are defined as $N$ (never), $O / M$ (once a month), $O / W$ (once a week), $D$ (Daily) and $S / D$ (Several times a day).

Over $90 \%$ of the participants sent and received email many times a day and about $73 \%$ read news content on the Web at the same frequency. $18 \%$ read news

\footnotetext{
${ }^{1}$ University of Cape Town

${ }^{2}$ Information and Communication Technologies for Development
} 


\begin{tabular}{|l|l|l|l|l|l|}
\hline Question & N & O/M & O/W & D & S/D \\
\hline Send and receive email. & $0 \%$ & $0 \%$ & $0 \%$ & $9 \%$ & $91 \%$ \\
\hline Use a Web browser. & $0 \%$ & $0 \%$ & $0 \%$ & $0 \%$ & $100 \%$ \\
\hline Read news articles on the Web. & $0 \%$ & $0 \%$ & $9 \%$ & $18 \%$ & $73 \%$ \\
\hline $\begin{array}{l}\text { Search for information on the Inter- } \\
\text { net. }\end{array}$ & $0 \%$ & $0 \%$ & $0 \%$ & $0 \%$ & $100 \%$ \\
\hline
\end{tabular}

Table 5.1: Usability study participants' content consumption habits.

\begin{tabular}{|l|l|l|l|l|l|}
\hline Question & $\mathbf{N}$ & $\mathbf{O} / \mathbf{M}$ & $\mathbf{O} / \mathbf{W}$ & $\mathbf{D}$ & $\mathbf{S} / \mathbf{D}$ \\
\hline $\begin{array}{l}\text { Comment on articles or Web pages e.g. } \\
\text { on blogs, YouTube etc. }\end{array}$ & $9 \%$ & $27 \%$ & $45 \%$ & $18 \%$ & $0 \%$ \\
\hline $\begin{array}{l}\text { Upload pictures or photos e.g. to Flickr, } \\
\text { Facebook etc. }\end{array}$ & $9 \%$ & $64 \%$ & $18 \%$ & $9 \%$ & $0 \%$ \\
\hline $\begin{array}{l}\text { Annotate content on a webpage i.e. } \\
\text { highlight and tag specific portions or a } \\
\text { page and add commentary. }\end{array}$ & $45 \%$ & $45 \%$ & $9 \%$ & $0 \%$ & $0 \%$ \\
\hline $\begin{array}{l}\text { Create articles e.g. on blogs or collabo- } \\
\text { rate on creating and editing documents } \\
\text { e.g. Google Docs etc. }\end{array}$ & $9 \%$ & $64 \%$ & $27 \%$ & $0 \%$ & $0 \%$ \\
\hline
\end{tabular}

Table 5.2: Usability study participants' content generation habits.

daily with the rest checking at least once a week. All the participants used a Web browser and carried out Web searches online several times a day as well.

None of the participants performed any of the listed content generation actions several times per day, with $18 \%$ commenting on articles and pages daily and $9 \%$ uploading photos. The majority of the participants rarely performed these actions. This means that they did them at least once per week or per month. $9 \%$ of users did not ever comment, upload or create articles online. It is also important to note that $45 \%$ did not ever annotate content on any webpage.

These results show that all the participants were conversant with the Web and that they predominantly used the Web to consume content as opposed to generating content. Since annotation is a content generation process, these results show that the pool of participants used for this part of the study was appropriate. This was because the objective of the study is to use gamified annotations to encourage content generation. 


\subsubsection{Usability Survey Analysis}

\section{Usefulness}

Graphed results for the questions asked on usefulness from the U.S.E. questionnaire can be viewed in Figure 5.1 below.

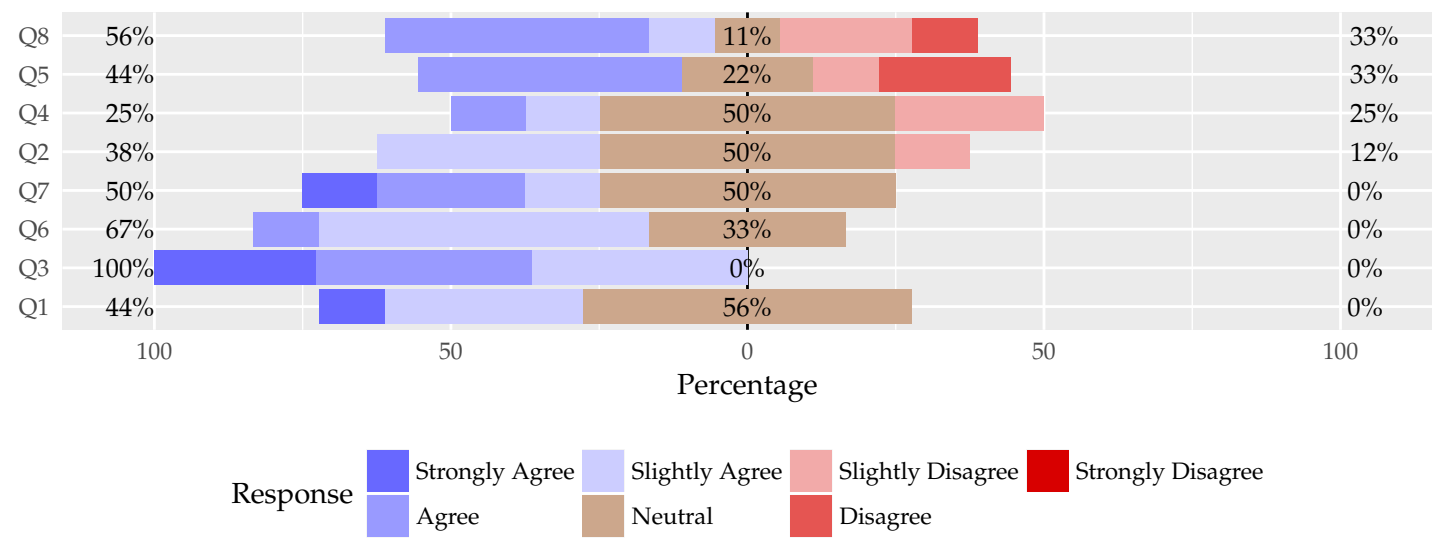

Figure 5.1: Graphed survey responses giving a measure of the usefulness of the usability testing prototype.

The questions were as follows: it helps me be more effective (Q1), it helps me be more productive (Q2), it is useful (Q3), it gives me more control over the activities in my life (Q4), it makes the things I want to accomplish easier to get done (Q5), it saves me time when I use it (Q6), it meets my needs (Q7) and it does everything I would expect it to do (Q8).

In each question, the responses show that the percentage of users in agreement exceeded those in disagreement, except in question Q4 where there was a tie at $25 \%$. All participants unanimously agreed that the system was useful, as shown in the responses to Q3.

Interestingly, unlike other sections in the U.S.E. questionnaire - such as ease of use, ease of learning and satisfaction - it was noted that a lot of the participants marked off questions in this section as 'neutral' or 'not applicable'. In some of these questions, up to $50 \%$ of the users were neutral.

Based on the results shown in Figure 5.1, it was concluded that the users found the system useful.

\section{Ease of Use}

Graphed results for the questions asked on ease of use from the U.S.E. questionnaire can be viewed in Figure 5.2. 


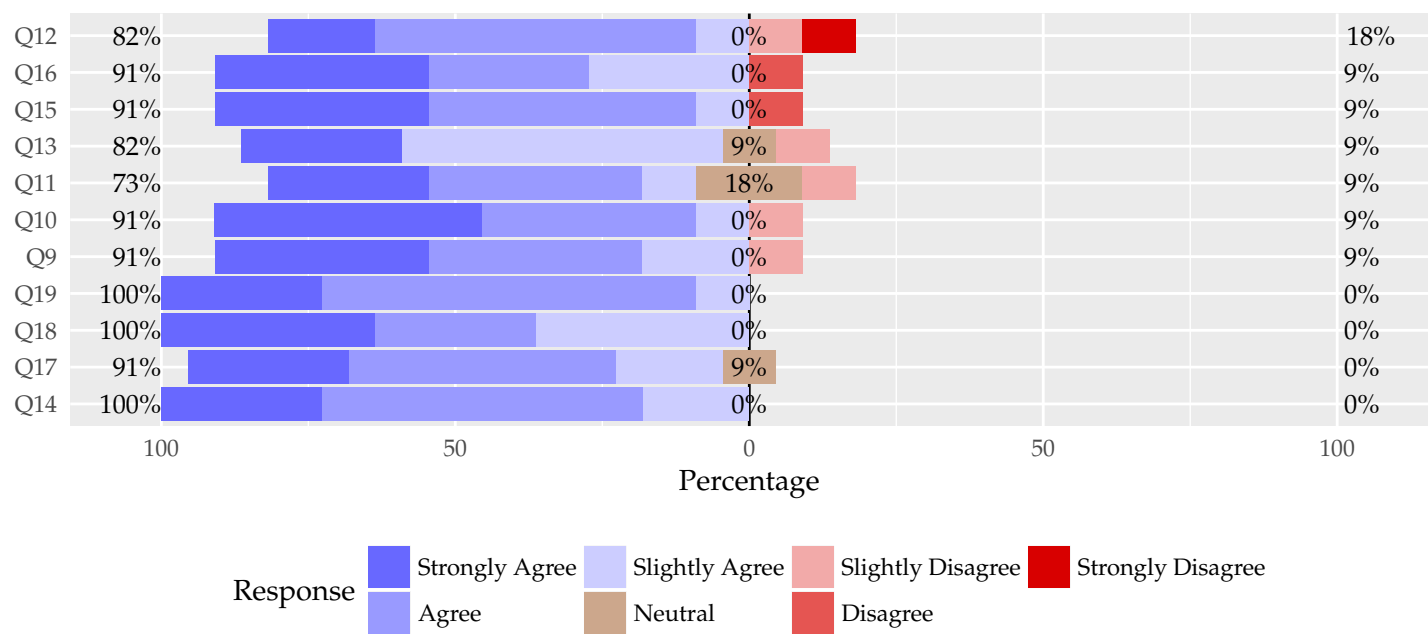

Figure 5.2: Graphed survey responses giving a measure of how easy it is to use the usability testing prototype.

The questions were as follows: it is easy to use (Q9), it is simple to use (Q10), it is user friendly $(Q 11)$, it requires the fewest steps possible to accomplish what I want to do with it (Q12), it is flexible (Q13), using it is effortless (Q14), I can use it without written instructions (Q15), I don't notice any inconsistencies as I use it (Q16), both occasional and regular users would like it (Q17), I can recover from mistakes quickly and easily (Q18) and I can use it successfully every time (Q19).

$91 \%$ found that the system was easy and simple to use and that they were able to use it without written instructions. A similar percentage agreed that the system did not exhibit any inconsistencies as they used it and that both occasional and regular users would like it.

All participants were able to recover from mistakes quickly and easily, use it effortlessly and successfully each time. Responses to the rest of the questions were similarly overwhelmingly positive.

Based on the results shown in Figure 5.2, it was concluded that the users found the system easy to use.

\section{Ease of Learning}

Graphed results for the questions asked on ease of learning from the U.S.E. questionnaire can be viewed in Figure 5.3.

The questions were as follows: I learned to use it quickly (Q20), I easily remember how to use it (Q21), it is easy to learn to use it (Q22) and I quickly became skilful with it (Q23). 


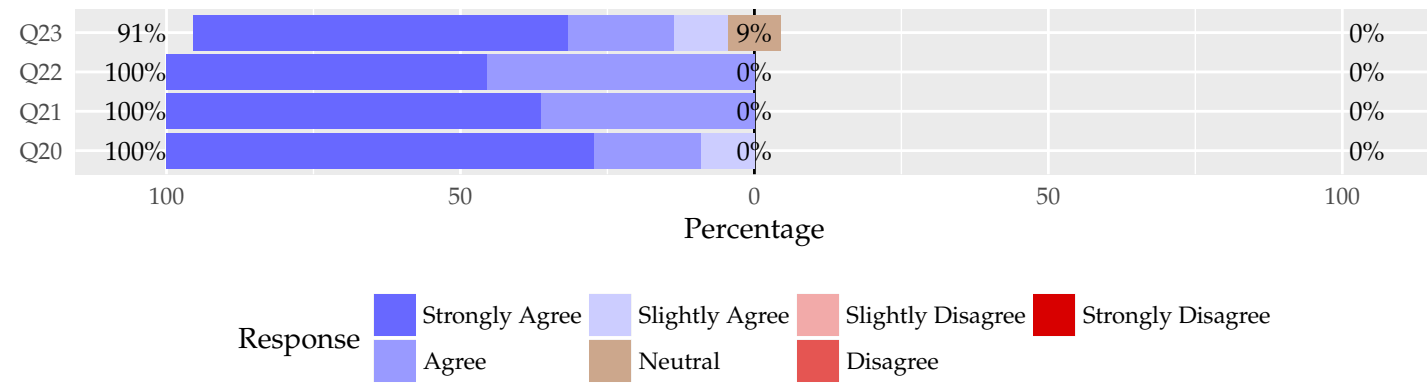

Figure 5.3: Graphed survey responses giving a measure of how easy it is to learn how to use the usability testing prototype.

All participants were able to learn how to use the tool quickly and easily and even remember how to use it. $91 \%$ agreed that they quickly became skilful at using it with only $9 \%$ neutral. None of the users expressed disagreement in any of the questions asked.

Based on the results shown in Figure 5.3, it was concluded that the users found it easy to get to learn how to use the system.

\section{Satisfaction}

Graphed results for the questions asked on satisfaction from the U.S.E. questionnaire can be viewed in Figure 5.4.

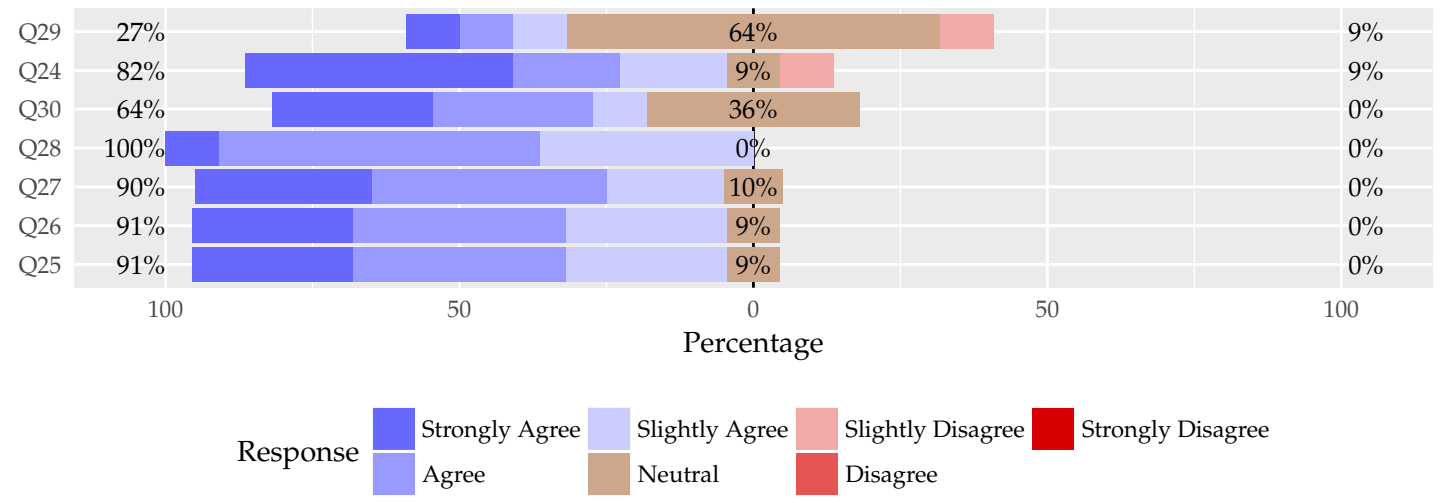

Figure 5.4: Graphed survey responses giving a measure of the users' satisfaction while using the usability testing prototype.

The questions were as follows: I am satisfied with it (Q24), I would recommend it to a friend (Q25), it is fun to use (Q26), it works the way I want it to work 
(Q27), it is wonderful (Q28), I feel I need to have it (Q29) and it is pleasant to use (Q30).

$91 \%$ of the participants agreed that it was fun using the system and that they would recommend it to a friend. $82 \%$ agreed that they were satisfied with the system with a majority of those strongly in agreement. $90 \%$ agreed that the system worked the way they wanted it to work. All participants agreed that it is a wonderful tool.

Interestingly, only $27 \%$ agreed that they felt that they needed the system (Q29). Despite this relatively low percentage, it was observed the cause was because a large percentage of the users were neutral $(64 \%)$.

Based on the results shown in Figure 5.4, it was concluded that the users were satisfied with the system.

\section{Written And Verbal Feedback}

In addition to Likert-scale type responses, users were encouraged to provide their own written comments and verbal feedback as they carried out the tasks.

- Positive Comments - Two participants said they liked the way the system looked and functioned, with one of them saying, "it seems to be straight forward".

The white feather widget on the image annotations was useful as it prodded the user to take further action. Another user also found the pen on the tooltip appropriate since they could associate the image of a pen with the action of writing or editing. One of the users commented on this visual aid, saying they found the tooltip to be very useful. See Figure 3.3 for the widgets.

Most users were able to eventually figure out how to add, edit and delete both text and image annotations. They took a while to annotate the first time but, once they got used to it, the interaction with the tool was seamless.

Some other users liked the threading feature of the annotations in the popup dialogue that would consist of annotations made on the same section of text. However, it is important to note that only those users who made annotations on the same section and on the same page got to see their annotations threaded since there was an issue persisting annotation when the page was refreshed. This was possible because the JavaScript is used to build the UI dynamically.

- Recommendations \& Other Comments - Some users experienced difficulty in finding the edit and delete buttons. The buttons were not always 
visible. The buttons would only be visible when the mouse was hovering over the popup image. Another user found that the popup would disappear too quickly when they tried to edit. One user commented, "someone must be a regular computer user to know what to do".

Text sub-annotations (where two or more annotations exist on the same portion of text) also were not easily visible because they were differentiated from each other using a shade of the yellow. This might have been an issue for people who have difficulties differentiating slight shades of the same colour apart. One user suggested that each sub-annotation be displayed in a different colour to better distinguish them. The system would get very confusing when there are multiple annotations because of the difficulty to distinguish yellow-on-yellow. Another user suggested that annotations from different authors should be displayed in different colours to distinguish them and also asked if it was possible for them to choose the highlight colour for their annotations.

In the case where we had threaded annotations, one of the users suggested that there should be a feature that would highlight the sub-annotation that the mouse is over on the thread of annotations. This approach would be able to show the user exactly which region the annotation in the thread is associated with.

Some users found the text annotation feature to be non-intuitive. It was not easy to know if the text was annotatable at the onset before you select text to annotate. The image annotation process was also found to be non-intuitive by some users. The user occasionally did not know how to annotate an image successfully without being instructed on the process. Some would click once and not drag to create the bounding box that defines the area that they want annotated. On one occasion a user tried to drag the annotation tooltip on the image to begin the annotation process.

On the image tagging interface, two users compared it to Facebook's approach. Unlike Facebook, the annotation widget did not predefine the size of the bounding box to which the user was going to add an annotation. One had to click and drag the mouse on the image to define the area of the bounding box. On release, the annotation widget would then appear and at this stage the user was expected to begin annotating or cancel, thereby starting the process from the beginning. Using this approach, there was no opportunity for the user to redefine the size of the bounding box or move it to a more suitable position on the image. Facebook, on the other hand, offered a more flexible approach. The tagging widget would add a bounding box of predefined size as soon as the user clicked on the image and then the 
user would be able to resize the box or move it to cover whatever area of the image they wanted to tag.

The written and verbal feedback from the users was taken into consideration when working on the parts of the system that exhibited inconsistent behaviour, such as the persisting of the annotations. Only feedback affecting the core functionality of the system was considered so as to stay within the scope of the study.

\subsection{Gamification Experiment Evaluation}

\subsubsection{Experimental Data}

The annotations used to evaluate the system in this experiment were from 112 participants who successfully registered with the Annotated Bleek \& Lloyd Collection within a period of 4 weeks.

\section{Distribution Of Users Based On Gamification Mode}

$56(50 \%)$ participants were allocated to the gamified category of users while the other $56(50 \%)$ participants were allocated to the un-gamified category of users, which is an equal distribution of users to each group.

\section{Distribution Of Users Based On Annotation Activity}

Out of the total 112 participants, only $25(22.32 \%)$ submitted annotations while the other $87(77.68 \%)$ did not submit any annotations. A majority of the users chose only to browse the digital heritage archive and not make any annotations. See Figure 5.5.

\section{Distribution Of Users Based On Annotation Type}

A total of 387 image and text annotations were made. 204 (52.71\%) of these annotations were image annotations while the other $183(47.29 \%)$ annotations were text annotations. The difference in the percentage of image and text annotations shows that users had no preference of one type of annotation over the other. See Figure 5.6.

\section{Users' Background And Previous Experience With Digital Archives}

31 users took the time to complete the system evaluation survey after using the digital heritage archive. 20 were women and 11 were men. The participants came 
Users who did not annotate

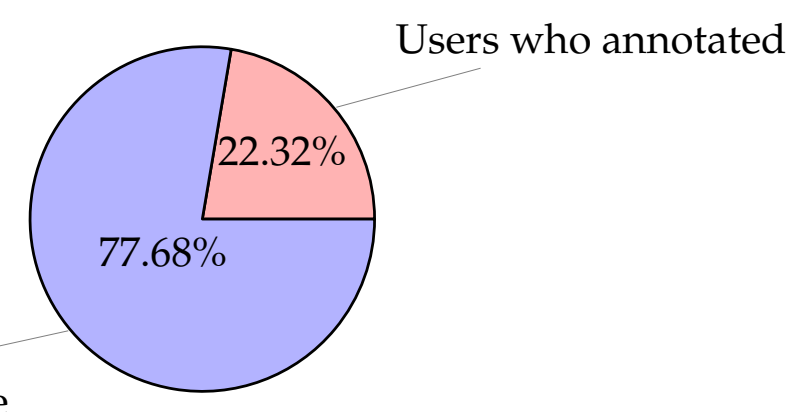

Figure 5.5: Distribution of 112 users by their annotation activity.

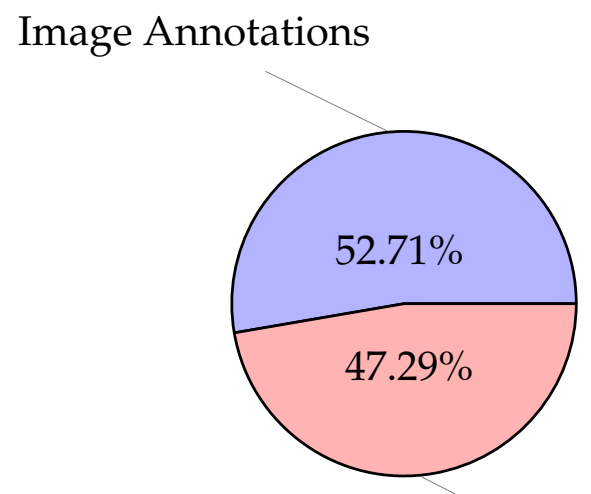

Text Annotations

Figure 5.6: Distribution of 387 submitted user annotations by type.

from diverse backgrounds with different degree levels, majors and year when they joined university. For both the gamified and un-gamified groups, over $80 \%$ of the users were not new to digital archives, however, for all the participants it was their first time using a digital heritage archive. See Table 5.3.

\begin{tabular}{|l|r|r|r|r|}
\hline Question & \multicolumn{3}{|c|}{ Gamified } & \multicolumn{2}{|c|}{ Un-gamified } \\
\cline { 2 - 5 } & $Y e s$ & No & Yes & No \\
\hline First time browsing a digital archive? & 3 & 13 & 3 & 12 \\
First time browsing one specific to heritage? & 16 & 0 & 15 & 0 \\
\hline
\end{tabular}

Table 5.3: Survey participants' previous history with digital archives. 


\subsubsection{Task Analysis}

\section{Rank Annotating Users In Order Of Points}

Sorting the annotating participants in order of the total points attained reveals a majority of gamified users. See Table 5.4. The total points for each user was calculated as shown in Equations 3.1, 3.2 and 3.3. It is important to note that only users in the gamified group could up-vote. This means that any up-votes that users in the un-gamified group were awarded were as a result of users in the gamified group up-voting their annotations.

\begin{tabular}{|r|r|r|r|r|l|}
\hline User ID & Annotations & Up-votes & Points & Rank & Mode \\
\hline 19 & 80 & 0 & 80 & 1 & Gamified \\
32 & 37 & 6 & 43 & 2 & Gamified \\
22 & 33 & 7 & 40 & 3 & Gamified \\
40 & 36 & 0 & 36 & 4 & Un-gamified \\
115 & 32 & 2 & 34 & 5 & Gamified \\
58 & 21 & 0 & 21 & 6 & Gamified \\
11 & 17 & 2 & 19 & 7 & Gamified \\
92 & 19 & 0 & 19 & 8 & Un-gamified \\
101 & 16 & 0 & 16 & 9 & Gamified \\
46 & 15 & 0 & 15 & 10 & Gamified \\
8 & 11 & 3 & 14 & 11 & Un-gamified \\
123 & 14 & 0 & 14 & 12 & Un-gamified \\
\hline 9 & 12 & 0 & 12 & 13 & Gamified \\
\hline 39 & 10 & 1 & 11 & 14 & Gamified \\
64 & 7 & 0 & 7 & 15 & Gamified \\
100 & 6 & 0 & 6 & 16 & Un-gamified \\
81 & 4 & 2 & 6 & 17 & Gamified \\
63 & 4 & 0 & 4 & 18 & Un-gamified \\
88 & 3 & 0 & 3 & 19 & Un-gamified \\
55 & 3 & 0 & 3 & 20 & Un-gamified \\
82 & 2 & 0 & 2 & 21 & Gamified \\
42 & 2 & 0 & 2 & 22 & Un-gamified \\
124 & 1 & 0 & 1 & 23 & Gamified \\
65 & 1 & 0 & 1 & 24 & Un-gamified \\
66 & 1 & 0 & 1 & 25 & Gamified \\
\hline & & 0 & & \\
\hline
\end{tabular}

Table 5.4: Ranked list of the 25 users who annotated in order of their total points scored calculated from images/text annotations and up-votes. The user ranked $13^{\text {th }}$ splits the results into two halves. 
Sorting the annotating participants in order of the total annotations they submitted also follows a similar trend. This is expected as the number of annotations made is directly proportional to the points obtained and the number of up-votes was not high.

Out of the 25 annotating participants on the list, $15(60 \%)$ are gamified users while the remaining $10(40 \%)$ are un-gamified users. 6 out of the $10(60 \%)$ ungamified users were found in the bottom half of the list and with points lower than 6, which is relatively low compared to the points of those users in the first half of the list. In fact, this is less than half the points scored by the lowest scorer in the first half.

Based on these results, we can conclude that the gamified users annotated more than un-gamified users.

\section{Relevance Of Annotation Content}

The annotations submitted could be categorised by content type into 2 groups: relevant and feedback annotations. 'Relevant' refers to those annotations whose content was directly related to or is a comment on the subject matter of the digital heritage archive. 'Feedback' refers to those annotations whose content was considered to be a message made to the owner of the digital heritage archive to communicate appreciation, feature requests or comment on design aspects of the archive. See Table 5.5.

\begin{tabular}{|l|r|r|r|}
\hline Category (Text Annotations) & Gamified & Un-gamified & Total \\
\hline Feedback & $106(68.8 \%)$ & $48(31.2 \%)$ & 154 \\
Relevant & $18(62.1 \%)$ & $11(37.9 \%)$ & 29 \\
\hline
\end{tabular}

\begin{tabular}{|l|r|r|r|}
\hline Category (Image Annotations) & Gamified & Un-gamified & Total \\
\hline Feedback & $7(70.0 \%)$ & $3(30.0 \%)$ & 10 \\
Relevant & $157(80.9 \%)$ & $37(19.1 \%)$ & 194 \\
\hline
\end{tabular}

Table 5.5: Breakdown of 387 annotations by relevance of annotation content.

194 of the $204(95.10 \%)$ image annotations were relevant to the subject matter. Text annotations had only 29 of the 183 (15.85\%) annotations submitted having relevant content with the remaining 154 annotations $(84.15 \%)$ used to provide feedback about the digital archive.

While relevance of the annotation content seemed to be affected by the type of annotation, it did not seem to be affected by gamification. 


\subsubsection{Survey Analysis}

\section{Effect of Gamification on User Motivations}

Four survey questions were targeted to find out what would contribute to each participants' motivations to: $a$ ) view more of the digital archive, $b$ ) revisit the digital archive, $c$ ) contribute to the digital archive and $d$ ) share the digital archive with others. In each action, 7 features were presented to the user for them to give feedback on which ones they felt compelled them to perform the respective action.

The features were as follows: having content on the digital heritage archive annotated with extra information (TQ1), having only your annotations visible at a time when browsing (TQ2), having everyone's annotations visible at the same time (including yours) when browsing (TQ3), receiving and being able to view points awarded to you for annotating (TQ4), being able to view other participant's scores (e.g. via visible scoreboard) (TQ5), receiving achievement badges based on points you have accumulated (e.g. 'Top Contributor', 'User of the year') (TQ6) and being able to view achievement badges assigned to other users (e.g. 'Top Contributor', 'User of the year') (TQ7).

The results for these 4 questions for both the gamified and un-gamified groups are shown in Figures 5.7, 5.8, 5.9 and 5.10 respectively.
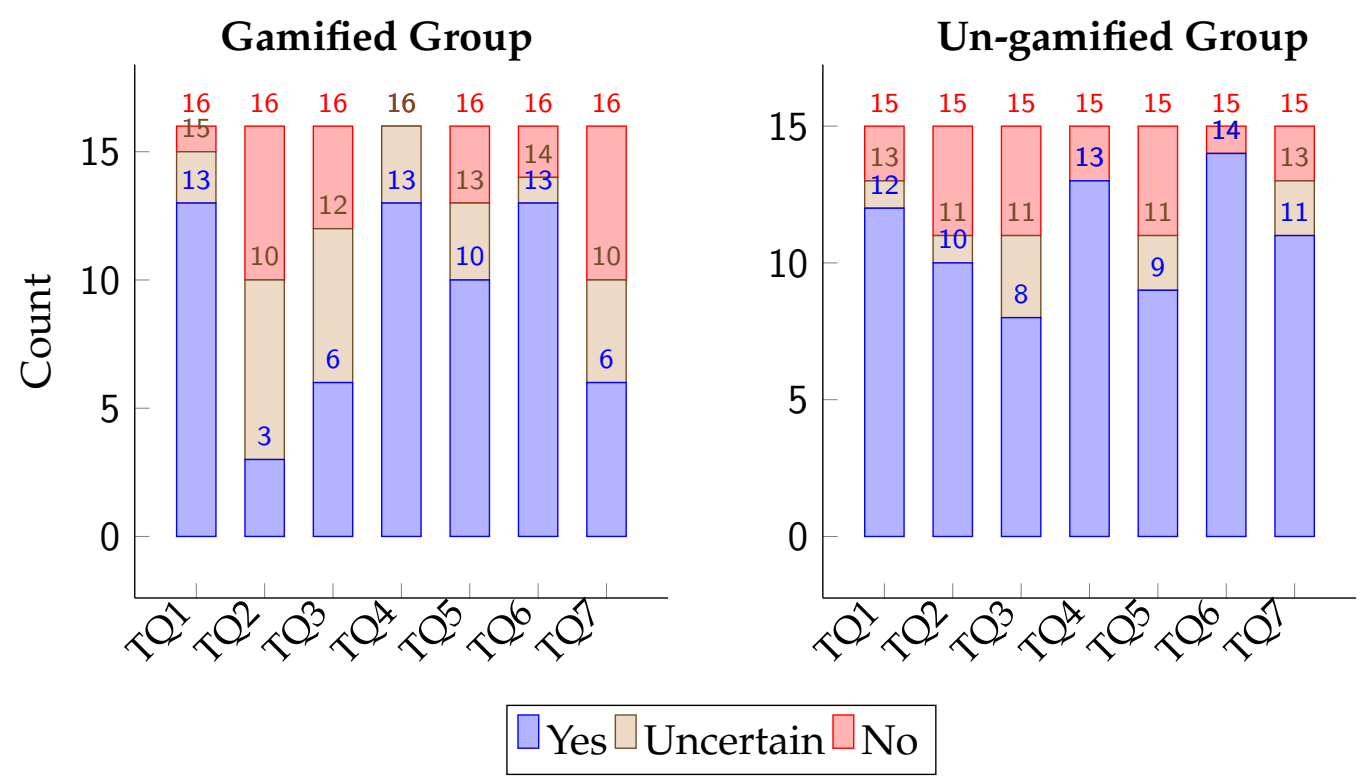

Figure 5.7: User feedback on features that promote or motivate viewing more of the digital heritage archive. 


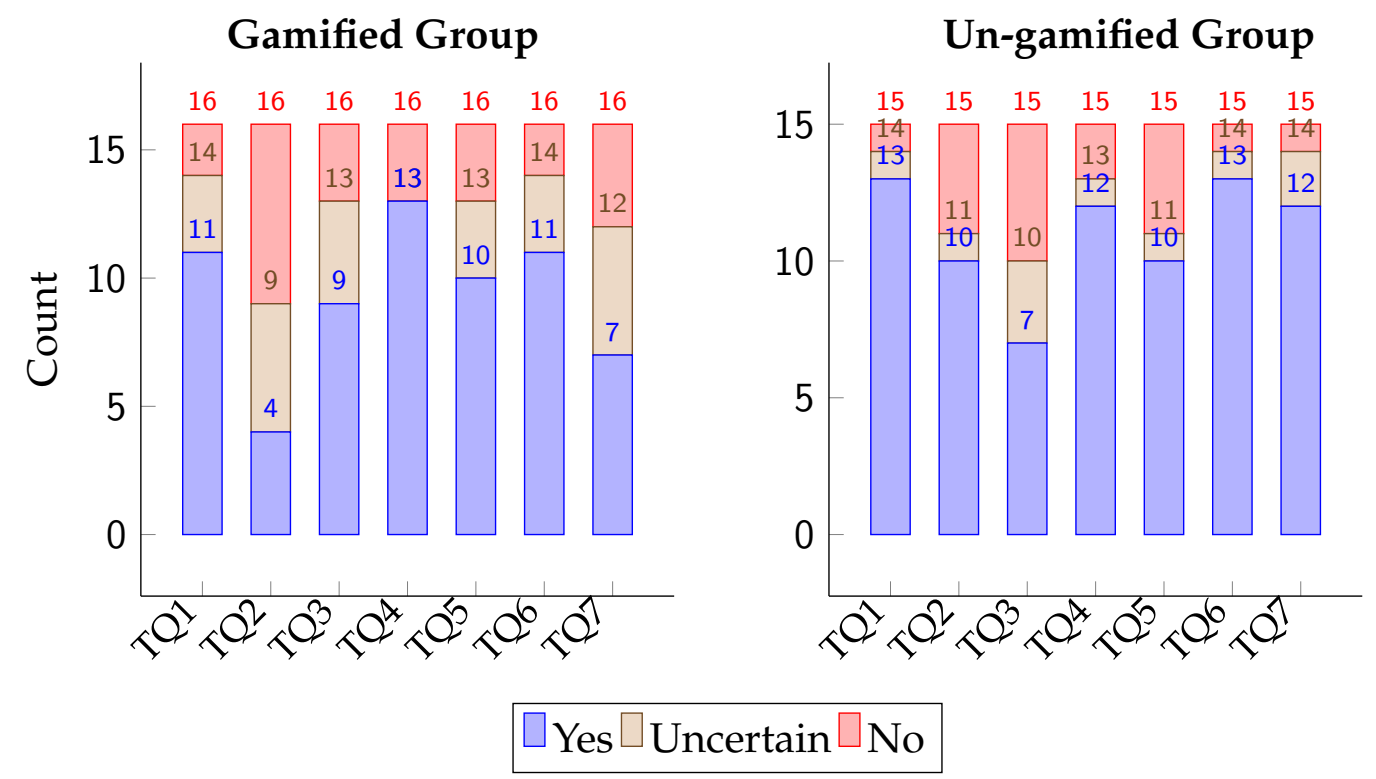

Figure 5.8: User feedback on features that promote or motivate contributing to the digital heritage archive.

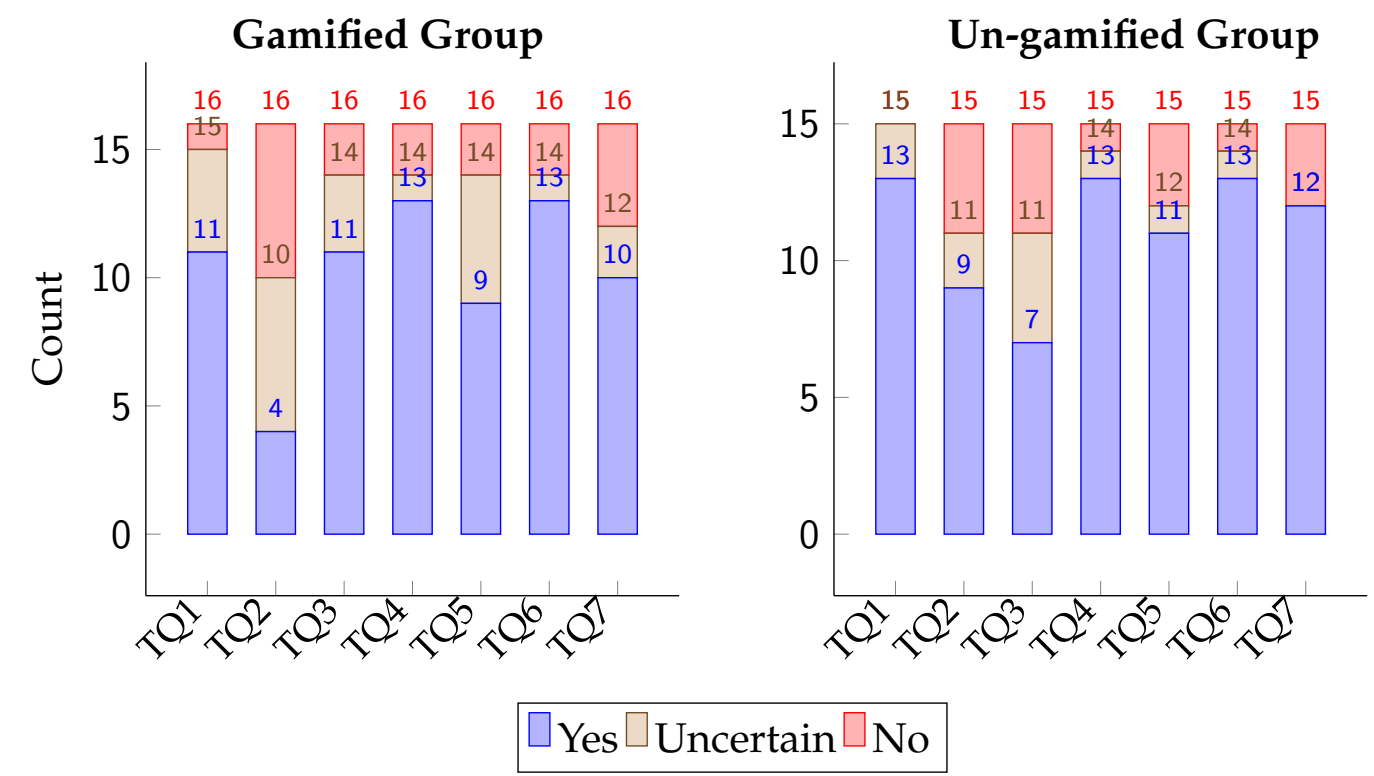

Figure 5.9: User feedback on features that promote or motivate revisiting of the digital heritage archive.

It was observed that the results showing user motivations to contribute more to the archive (Figure 5.8) or revisit the archive (Figure 5.9) had similar trends to the results showing user motivations to view more of the archive (Figure 5.7). 


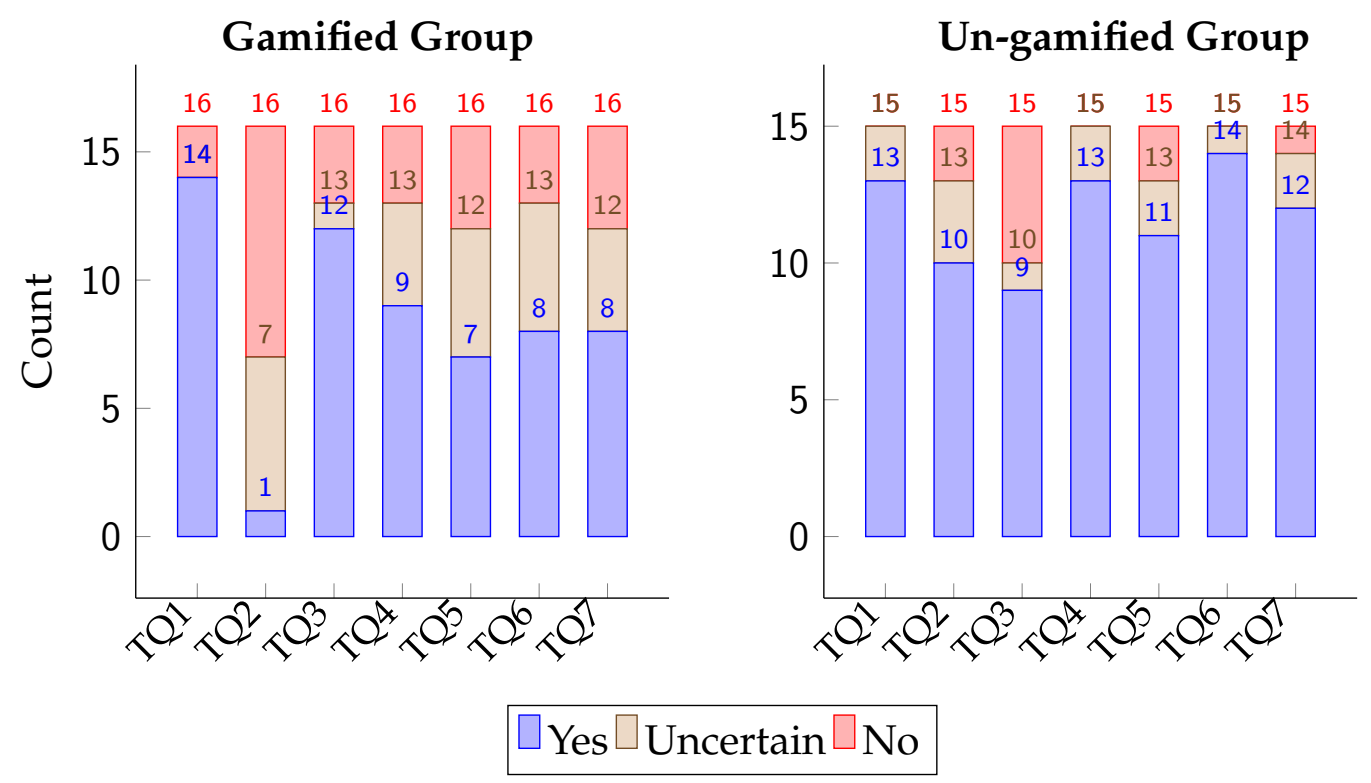

Figure 5.10: User feedback on features that promote or motivate sharing the digital heritage archive with others.

Results showing user motivations to share the digital heritage archive with others slightly varied in the gamified group but were consistent with the rest for the ungamified group (compared to the rest) (Figure 5.10).

In each instance, the results for $T Q 1, T Q 4, T Q 5, T Q 6$, and $T Q 7$ showed a consistent and positive feedback from the majority of users for both gamified and un-gamified groups. These show that features highlighted by each question contributes to the users' motivations because a majority of the users answered 'Yes'.

Preferences for both groups on TQ2 and TQ3 diverged. We noted that users in the gamified group preferred having everyone's annotations visible at the same time while users in the un-gamified group preferred only having the annotator's annotations visible.

We found that a relatively higher number of gamified users $T Q 7$ answered 'No' when asked if achievement badges would contribute to their motivation to view more of the archive compared to the rest of the aspects of user engagement. Further study is required to understand why.

\section{Effect of Gamification on Usability}

Questions EOS1 to EOS4 listed in Table 4.6 were used to investigate the usability of the system. EOS1 focused on the signup/login functionality, EOS2 on the creation of image annotations, EOS3 on the creation of text annotations and EOS4 on the browsing of the archive. The results of the usability survey questions are 
presented in Figures 5.11 and 5.12.

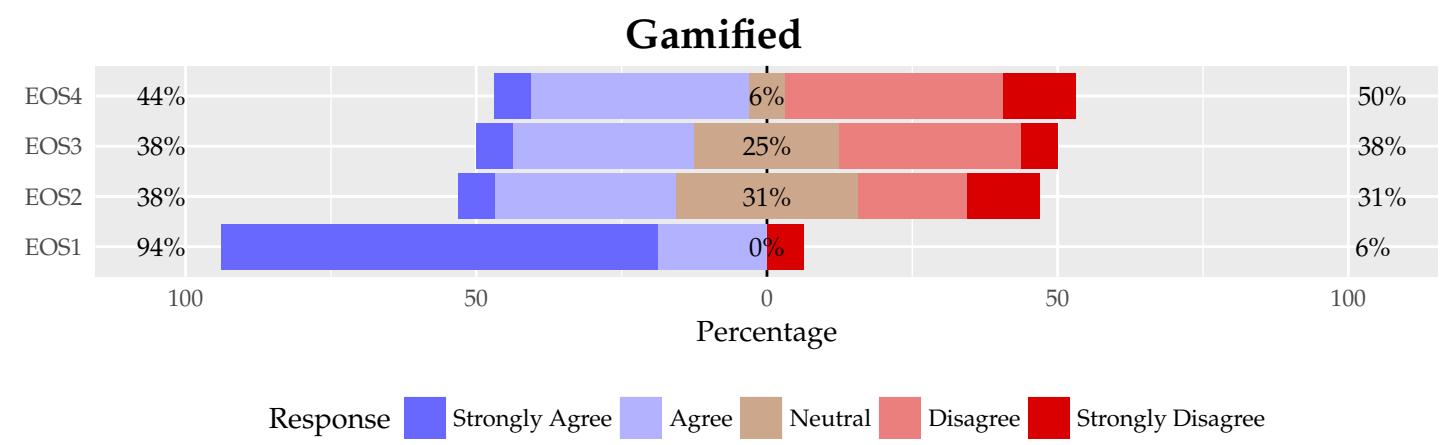

Figure 5.11: Survey responses giving a measure of the ease of use of the system according to gamified users.

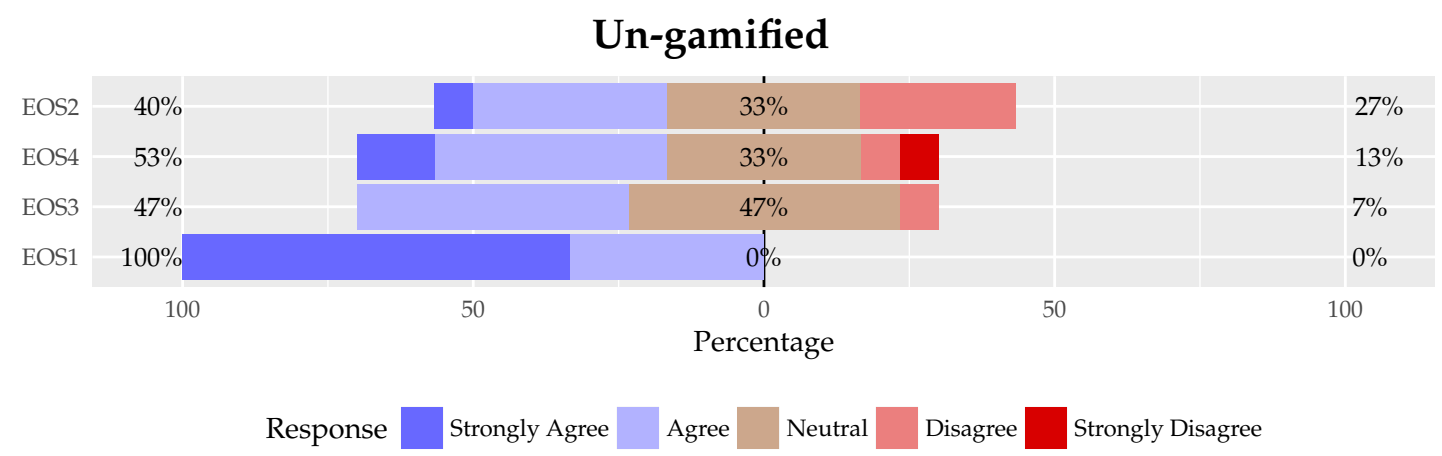

Figure 5.12: Survey responses giving a measure of the ease of use of the system according to un-gamified users.

For both the gamified and un-gamified groups, over $90 \%$ agreed that it was easy to sign up and log in into the system. This shows that both groups had no trouble getting into the system.

For the un-gamified group, a majority of users agreed that the creation of image annotations, creation of text annotations and browsing the archive was easy to do $(40 \%, 47 \%$ and $53 \%$ respectively). The gamified group on the other hand had greater numbers of users disagreeing and also had slightly lower percentages of users agreeing when compared to the un-gamified group.

These results show that users in the gamified group found it harder to use the system after logging in. Figure 5.11 hints that gamification could be introducing slight complexity to the system for the gamified users, causing them to find it more difficult to use the system. 


\section{User Feedback}

The users of the enhanced digital heritage archive were encouraged to give open feedback in the survey taken after interacting with the system. There was feedback from both gamified and un-gamified groups of users on both its positive and negative aspects.

Some comments were on the annotation functionality of the enhanced digital heritage archive. On the positive aspects, two users commented on the usefulness of being able to see other users' comments and points of view. Another user said they liked the ability to comment on any piece of text or section of an image. On the negative aspects, one of the users requested that a better system be developed to submit and display the annotations.

The rest of the feedback submitted focused on other aspects of the enhanced digital heritage archive that were neither related to the annotation nor gamification elements. These are: i) the amount and structure of content available, ii) the type and size of the font used and iii) the user interface of the original digital heritage archive. See Appendix B.1 and Appendix B.2 for a list of all the feedback.

\subsection{Summary}

We were able to carry out a usability survey of our system and the system was found to be useful, easy to use, easy to learn and satisfactory as per its intended objective.

The gamification experiment involved having a larger pool of users use the digital heritage archive with the annotation functionality built it. We evenly split the users into two groups. One group had gamification features made available to them while the other had the gamification features turned off.

From the results we observed that:

1. About a quarter of the users made annotations.

2. There was an almost equal number of text and image annotations.

3. Gamified users ranked higher in the number of points earned from annotation activity when compared to un-gamified users.

4. The relevance of annotations made varied greatly based on the type of content that was being annotated i.e. text versus images.

After the users got to use the enhanced digital heritage archive they were requested to take part in a survey to get feedback on their experience. The survey investigated their motivations for engagement, their view on the usability of the 
system and it was also an opportunity for them to give open feedback on their experience.

The results showed that both gamified and un-gamified users agreed that gamified annotation features would contribute to their motivations to engage. They also showed that gamified users preferred viewing everyone's annotations at the same time while un-gamified users preferred viewing only their annotations.

Despite the now proven benefits of gamified annotations, it was evident from the feedback survey that gamified annotations introduce some level of complexity. For example, during the usability study, we noticed that some users took time to discover the annotations features. Regardless, these issues did not hinder the functionality of the system.

We believe that the experiment was a success and that we were able to show that gamified annotations do improve the experience on a digital heritage archive. They promote engagement and they can be that layer atop fixed content that can foster the transference of knowledge between the various groups of users who visit digital heritage archives. 


\section{Chapter 6}

\section{Conclusion}

\section{Contents}

6.1 Overview . . . . . . . . . . . . . . . 120

6.2 Answers to Research Questions . . . . . . . . . . . . 120

6.2.1 Quantity of Annotations . . . . . . . . . . . . . . . . 120

6.2.2 Quality of Annotations . . . . . . . . . . . . . . . . 120

6.2.3 Appropriate for Digital Heritage Archives . . . . . . . 121

6.3 Other Observations . . . . . . . . . . . . . . . . . 121

6.3 .1 User Habits . . . . . . . . . . . . . . . . . . . . . . . . 121

6.3.2 Annotations as a Feedback Mechanism . . . . . . . . . . 121

6.3.3 Ease of Implementation . . . . . . . . . . . . . . . . 122

6.4 Contributions . . . . . . . . . . . . . . . . . . 123

6.5 Future Work . . . . . . . . . . . . . . . . . . . 123

6.5.1 Display of Multiple Annotations . . . . . . . . . . . . 123

6.5.2 Moderation of Annotations . . . . . . . . . . . . . . . . 124

6.5.3 Supporting Discourse in Annotations . . . . . . . . . . . 124

6.5.4 Annotating Other Types of Content . . . . . . . . . . . 124 


\subsection{Overview}

In this research, we aimed to investigate the feasibility of gamification coupled with annotations as a way to enhance digital heritage archives using a system that opens up their contents to a larger target audience and provides more interactivity and engagement.

In section 6.2 we summarise our understanding of the results in view of the guiding research questions. We then highlight our contributions in section 6.4 and suggest possible future work in section 6.5 .

\subsection{Answers to Research Questions}

The research questions sought to investigate whether: i) gamified annotations are an appropriate approach for digital heritage archives and; ii) if the gamification of annotations improved the number and the quality of annotations submitted to a digital heritage archive .

\subsubsection{Quantity of Annotations}

From our results we were able to conclude that gamified annotations encourage users to engage more with the content of the archive as well as promote and motivate them to view more of, contribute to, revisit and share the archive with others, without affecting the experience and ease of use of the digital heritage archive.

This was demonstrated in our gamification experiment where it was observed that the gamified users garnered a significantly higher number of points when compared to their un-gamified counterparts. It was also observed that when ranked by number of points, they dominated the top half of the list. See section 5.4.2 and Table 5.4.

\subsubsection{Quality of Annotations}

When assessing the quality of annotations, we settled on the definition of a quality annotation as an annotation that is deemed relevant to the subject matter of the digital heritage archive. Therefore, as per this definition we investigated the relationship of high quality annotations with some of the variables in our gamification experiment.

We found that there was no relationship between high quality annotations and gamification. However, we observed that there was a relationship between the high quality annotations and the type of annotation. For example, the majority 
of the image annotations had an annotation body that was directly related to the image, section of the image or subject matter of the digital heritage archive while the converse held true with the text annotations. See section 5.4.2 and Table 5.5.

\subsubsection{Appropriate for Digital Heritage Archives}

From the survey of our gamification experiment, we were able to come to the conclusion that users were receptive to gamified annotations on the digital heritage archive. When queried about which features contributed to their motivations, a majority of the users agreed that the gamification and annotation features would motivate them to view more, revisit, contribute to and share the digital archive with others. See Figures 5.7, 5.8, 5.9 and 5.10.

We were also able to demonstrate that annotations can be easily added to an existing digital heritage archive, without requiring significant changes to the archive's design. The gamified annotations was implemented as a layer above the fixed content of the archive.

\subsection{Other Observations}

\subsubsection{User Habits}

When looking at the content consumption and content generation habits of the pool of users in our usability experiment, we came to the conclusion that most users would lean towards a content-consumption role as opposed to a contentgeneration role. This was clear in our usability survey where we queried the habits of the respondents and found that they spent most of their time on the Web consuming content.

We could see this trend manifest itself in the gamification experiment as well. About a quarter of the pool of users active on the system ended up submitting annotations. This hints at the real possibility that, in a majority of the digital heritage archives where this could be applied, only a subset of users would eventually make use of the annotation feature if implemented.

\subsubsection{Annotations as a Feedback Mechanism}

Our expectation was that at the end of the experiment the users who annotated would make annotations about the content or subject matter of the digital heritage archive. We were subtly surprised at the number of annotations that were made as a means to provide feedback. These were seemingly targeted to whoever the annotator thought was responsible for the content of the digital heritage 
archive. We see this as an unexpected effect that is welcome and can have positive implications for the quality of a digital heritage archive.

In the case of positive feedback, this could give insight to the features or content of the archive that the viewers and annotators respond well to. This insight can then be used to know what to promote more or what type of content the users respond best to. In the case of negative feedback, this can be used to continuously address the pain points of the digital heritage archive. Some examples include: incorrect spelling, incoherent explanation and difficult navigation. The cumulative effect of addressing both is that the archive keeps working towards what works well with the users and improves on what does not.

Another observation was that, unlike a typical contact form, these annotations are able to provide very precise context to the exact location of what the feedback is targeting. For example, instead of a user describing in words where a problem is, they can implicitly show where it is since the annotation has inherent location information.

\subsubsection{Ease of Implementation}

Through the experimental prototype, we were able to prove that it was trivial to implement gamified annotations in an existing digital heritage archive. This was done without requiring significant changes to its design. In our case, the underlying structure of the digital heritage archive remained largely unmodified and most of the technical work was in implementing the annotation store that would be external to the digital heritage archive anyway.

The modification made on the digital heritage archive in our case only involved adding a JavaScript script to the HTML header on each Web page of the archive. Since digital archives can be powered either by a content management framework that generates dynamic Web pages or by scripts that generate static Web pages, it is therefore possible to update the underlying generator to easily plug in a reference to the aforementioned client-side JavaScript component.

We also found that the design choice to have the annotation store as an external service allows one to have an annotation store that may be serving more than one digital heritage archive at the same time. This provides greater flexibility, eases implementation and reduces cost in the case of supporting more that one digital heritage archive.

We acknowledge that the requirements of an annotation store may vary depending on the project in reality. However, this is not an impediment to implementation in any way if one can provision their own annotation store. This is because annotation standards and implementation libraries seem to define the structure of annotations coming in from the client and therefore do not place re- 
strictions on how the annotation stores themselves work. Therefore, open-source store implementations such as the one developed for this study may be cloned and adapted to fit into whichever use case the implementer desires.

\subsection{Contributions}

The annotation store developed as part of this study has been open-sourced on GitHub $^{1}$. It is a developed as a Rails engine ${ }^{2}$ so that it is trivial to integrate into any supported Rails application.

It was based off the Annotatelt ${ }^{3}$ store reference implementation, which only supported text annotations. We therefore went further with our implementation and added support for image annotations, which followed the structure described in sub-section 3.6.2.

Effort was made to include automated testing of the core functionality of the library against a matrix of different versions of its dependencies, namely: 5 versions of Ruby, 3 versions of Rails and 2 different relational databases ${ }^{4}$.

See Appendix A for more details.

\subsection{Future Work}

In our research we focused on the implementation details of adding gamified annotations to a digital heritage archive and then we studied its immediate consequences. From our results, it is clear that there are areas that we did not cover that merit further research.

\subsubsection{Display of Multiple Annotations}

In section 6.2 we highlighted the complexities that immediately arose when the annotation system was presented to a large pool of users. The display of multiple annotations is certainly an issue that needs to be addressed if any annotation system is to be usable with such a large number of users, if not more.

Our study settled on the presentation mode used by Annotator. In the case of text, the approach was to differentiate annotations over the same content using

\footnotetext{
${ }^{1}$ https://github.com/itsmrwave/annotator_store-gem

${ }^{2} \mathrm{~A}$ Rails engine is a special type is Ruby library (also known as a gem) that can provide the packaged Rails app functionality to its host application.

${ }^{3}$ http: //annotateit.org

${ }^{4}$ Ruby version 1.9.3, 2.0.0, 2.1.0, 2.1.1 and 2.1.2. Rails version 4.0.x, 4.1.x and 4.2.x. MySQL and PostgreSQL databases.
} 
different shades of the same colour. This worked well when there were a few annotations made on the same chunks of text, but quickly proved not to be scalable when more than three annotation highlights overlapped. What we observed was that the resulting shade of the highlight did not adequately distinguish the different annotations made to the user.

\subsubsection{Moderation of Annotations}

In our setup, we had no moderation system to vet the annotations before they are made public on the digital heritage archive. A good case for moderation is to make sure that annotations made are always on topic. However, moderation requires a human to act as the moderator.

As mentioned in the motivating case study in section 1.4, Genius has an interesting approach to moderation where moderators are selected from the annotators themselves. The election of an annotator as a moderator is based on their annotation activity. This presents interesting dynamics for gamified annotations that should be investigated further.

\subsubsection{Supporting Discourse in Annotations}

An alternative implementation of an annotation system would involve linking annotations together into a threaded conversation. For example, on the Genius platform, the first annotation that is approved after moderation becomes the primary annotation. Any subsequent annotation on the same text is regarded as complementary to the primary annotation. With this approach there would be no need to overlay annotations on each other using shaded highlights as we had in our current implementation.

A second study could possibly explore the implications of this design, especially in light of the fact that there were no conversations amongst annotators in our study. Admittedly, the user-interface designed did not provide the users with the opportunity to reply to another annotator's annotations.

However, we were cognisant of the fact that such an annotation system is not trivial to develop and could possibly increase development time significantly.

\subsubsection{Annotating Other Types of Content}

Our study was limited to annotation of text and images, however, digital heritage archives sometimes may consist of other types of content such as: video, audio and even $3 \mathrm{D}$ tours. It is possible that users would respond differently to 
these other types of content and even exhibit differing annotation habits as was demonstrated in the gamification experiment.

We found it fit to exclude these other content types because they would significantly increase development time. Based on our background research, we noted that annotation of other content types is not trivial, especially when compared to annotations on text and images. 



\section{Bibliography}

[1] 1641 Depositions (Trinity College Dublin, MSS 809-841), 2007. URL http: //1641.tcd.ie/. [Accessed Online: 2015-06-25].

[2] IPSA (Imaginum Patavinae Scientiae Archivum), 2007. URL http://ipsa. dei . unipd.it. [Accessed Online: 2015-06-25].

[3] The Digital Bleek and Lloyd Collection, 2007. URL http: //lloydbleekcollection.cs.uct.ac.za. [Accessed Online: 2014-0731].

[4] Amaya Home Page, December 2012. URL https://www.w3.org/Amaya/. [Accessed Online: 2016-10-23].

[5] CULTURA (Cultivating Understanding and Research through Adaptivity), 2013. URL http: //www . cultura-strep.eu/. [Accessed Online: 2015-06-25].

[6] eXtensible Access Control Markup Language (XACML) Version 3.0, January 2013. URL http://docs.oasis-open.org/xacml/3.0/xacml-3.0core-spec-en.html. [Accessed Online: 2016-10-23].

[7] Annotator - Annotating the Web, 2014. URL http://annotatorjs.org/. [Accessed Online: 2014-07-22].

[8] About Genius I Genius, July 2014. URL http://meta.genius .com/Geniusfounders-introducing-geniuscom-annotated. [Accessed Online: 2014-1005].

[9] What is an Editor? I Genius, June 2014. URL http://meta.genius.com/ Genius-what-is-an-editor-annotated. [Accessed Online: 2014-10-06].

[10] What is an Moderator? I Genius, 2014. URL http://meta.genius.com/ Genius-what-is-a-moderator-annotated. [Accessed Online: 2014-10-06].

[11] What is an Regulator? I Genius, May 2014. URL http: //meta.genius .com/ Genius-what-is-a-regulator-annotated. [Accessed Online: 2014-10-06]. 
[12] JSON-LD 1.0, January 2014. URL http://www .w3.org/TR/json-ld/. [Accessed Online: 2016-06-11].

[13] Open Annotation Community Group, 2014. URL http://www.w3.org/ community/openannotation/. [Accessed Online: 2014-05-06].

[14] Resource Description Framework (RDF), March 2014. URL https://www. w3. org/RDF/. [Accessed Online: 2016-10-23].

[15] Storage - Annotator 1.2.9 Documentation, 2015. URL http://docs. annotatorjs.org/en/v1.2.x/storage.html. [Accessed Online: 2015-0105].

[16] Cross-Origin Resource Sharing, 2015. URL http://www.w3.org/TR/2014/ REC-cors-20140116/. [Accessed Online: 2015-02-19].

[17] Web Annotation Data Model, March 2016. URL http://www.w3.org/TR/ 2016/WD-annotation-model-20160331/. [Accessed Online: 2016-06-11].

[18] jabber.org - the original XMPP instant messaging service, 2016. URL http: //www. jabber. org. [Accessed Online: 2016-10-23].

[19] Shibboleth, 2016. URL https://shibboleth. net. [Accessed Online: 201610-23].

[20] M. Agosti and N. Ferro. Context: Nature, Impact, and Role: 5th International Conference on Conceptions of Library and Information Sciences, CoLIS 2005, Glasgow, UK, June 4-8, 2005. Proceedings, chapter Annotations as Context for Searching Documents, pages 155-170. Springer Berlin Heidelberg, Berlin, Heidelberg, 2005. ISBN 978-3-540-32101-9. doi: 10.1007/11495222_13. URL http://dx.doi.org/10.1007/11495222_13.

[21] M. Agosti and N. Ferro. A formal model of annotations of digital content. ACM Transactions on Information Systems (TOIS), 26(1), November 2007. ISSN 1046-8188. doi: 10.1145/1292591.1292594. URL http://doi.acm.org/10. $1145 / 1292591.1292594$.

[22] M. Agosti, N. Ferro, I. Frommholz, and U. Thiel. Annotations in digital libraries and collaboratories - facets, models and usage. In Research and Advanced Technology for Digital Libraries, volume 3232 of Lecture Notes in Computer Science, pages 244-255. Springer Berlin Heidelberg, 2004. ISBN 978-3540-23013-7. doi: 10.1007/978-3-540-30230-8_23. URL http://dx.doi.org/ $10.1007 / 978-3-540-30230-8 \_23$. 
[23] M. Agosti, H. Albrechtsen, N. Ferro, I. Frommholz, P. Hansen, N. Orio, E. Panizzi, A. M. Pejtersen, and U. Thiel. DiLAS: a Digital Library Annotation Service. In IWAC, pages 91-101, 2005. URL http://www. is. informatik. uni-duisburg.de/bib/pdf/ir/Agosti_etal_05.pdf.

[24] M. Agosti, N. Ferro, E. Panizzi, and R. Trinchese. Annotation as a support to user interaction for content enhancement in digital libraries. In Proceedings of the Working Conference on Advanced Visual Interfaces, AVI '06, pages 151-154, New York, NY, USA, 2006. ACM. ISBN 1-59593-353-0. doi: 10.1145/1133265. 1133296. URL http://doi . acm.org/10.1145/1133265.1133296.

[25] M. Agosti, T. Coppotelli, N. Ferro, and L. Pretto. Asian Digital Libraries. Looking Back 10 Years and Forging New Frontiers: 10th International Conference on Asian Digital Libraries, ICADL 2007, Hanoi, Vietnam, December 10-13, 2007. Proceedings, chapter Annotations and Digital Libraries: Designing Adequate Test-Beds, pages 150-159. Springer Berlin Heidelberg, Berlin, Heidelberg, 2007. ISBN 978-3-540-77094-7. doi: 10.1007/978-3-540-77094-7_23. URL http://dx.doi.org/10.1007/978-3-540-77094-7_23.

[26] M. Agosti, O. Conlan, N. Ferro, C. Hampson, and G. Munnelly. Interacting with digital cultural heritage collections via annotations: The CULTURA approach. In Proceedings of the 2013 ACM Symposium on Document Engineering, DocEng '13, pages 13-22, New York, NY, USA, 2013. ACM. ISBN 978-1-45031789-4. doi: 10.1145/2494266.2494288. URL http://doi . acm.org/10.1145/ 2494266.2494288.

[27] M. Agosti, N. Ferro, N. Orio, and C. Ponchia. CULTURA outcomes for improving the user's engagement with cultural heritage collections. Procedia Computer Science, 38:34-39, 2014. ISSN 1877-0509. doi: 10.1016/j.procs. 2014.10.007. URL http://www.sciencedirect.com/science/article/pii/ S1877050914013672.

[28] M. Ames and M. Naaman. Why we tag: Motivations for annotation in mobile and online media. In Proceedings of the SIGCHI Conference on Human Factors in Computing Systems, CHI '07, pages 971-980, New York, NY, USA, 2007. ACM. ISBN 978-1-59593-593-9. doi: 10.1145/1240624.1240772. URL http://doi.acm.org/10.1145/1240624.1240772.

[29] M. Anderka and B. Stein. A breakdown of quality flaws in Wikipedia. In Proceedings of the 2nd Joint WICOW/AIRWeb Workshop on Web Quality, WebQuality '12, pages 11-18, New York, NY, USA, 2012. ACM. ISBN 978-1-4503-12370. doi: 10.1145/2184305.2184309. URL http://dl .acm.org/citation.cfm? $i d=2184305.2184309$. 
[30] A. F. Aparicio, F. L. G. Vela, J. L. G. Sánchez, and J. L. I. Montes. Analysis and application of gamification. In Proceedings of the 13th International Conference on Interacción Persona-Ordenador, INTERACCION '12, pages 17:1-17:2, New York, NY, USA, 2012. ACM. ISBN 978-1-4503-1314-8. doi: 10.1145/2379636. 2379653. URL http://dl . acm.org/citation. cfm?doid=2379636 2379653.

[31] E. Bailey, S. Lawless, A. O'Connor, S. Sweetnam, O. Conlan, C. Hampson, and V. Wade. CULTURA: supporting enhanced exploration of cultural archives through personalisation. In Proceedings of the 2nd International Conference on Humanities, Society and Culture, ICHSC, 2012. URL http://www.tara.tcd.ie/bitstream/handle/2262/67053/ ICSHSC_camera_ready . pdf? sequence $=1$.

[32] I. Bogost. Why gamification is bullshit 2. The Gameful World: Approaches, Issues, Applications, page 65, 2015. URL https://goo.gl/iZhEaO.

[33] P. Bottoni, R. Civica, S. Levialdi, L. Orso, E. Panizzi, and R. Trinchese. MADCOW: A multimedia digital annotation system. In Proceedings of the Working Conference on Advanced Visual Interfaces, AVI '04, pages 55-62, New York, NY, USA, 2004. ACM. ISBN 1-58113-867-9. doi: 10.1145/989863.989870. URL http://doi.acm.org/10.1145/989863.989870.

[34] P. Bottoni, S. Levialdi, A. Labella, E. Panizzi, R. Trinchese, and L. Gigli. Madcow: A visual interface for annotating web pages. In Proceedings of the Working Conference on Advanced Visual Interfaces, AVI '06, pages 314-317, New York, NY, USA, 2006. ACM. ISBN 1-59593-353-0. doi: 10.1145/1133265. 1133331. URL http://doi .acm.org/10.1145/1133265.1133331.

[35] H. Brocks, A. Stein, U. Thiel, I. Frommholz, and A. Dirsch-Weigand. How to incorporate collaborative discourse in cultural digital libraries. In Proceedings of the ECAI 2002 Workshop on Semantic Authoring, Annotation \& Knowledge Markup (SAAKM02), Lyon, France, 2002. URL http://www. is. informatik. uni-duisburg.de/bib/pdf/ir/Brocks_etal_02.pdf.

[36] C. Chen and C. Chen. Problem-based learning supported by digital archives: Case study of Taiwan libraries' history digital library. The Electronic Library, 28(1):5-28, 2010. doi: 10.1108/02640471011005414. URL http://dx.doi . org/10.1108/02640471011005414.

[37] D. Chisnell. Handbook of Usability Testing: How to Plan, Design, and Conduct Effective Tests. John Wiley \& Sons, 2nd edition, 2008. ISBN 9780470185483. URL http://eu.wiley.com/WileyCDA/WileyTitle/ productCd-0470185481.html. 
[38] E. L. Deci, R. Koestner, and R. M. Ryan. A meta-analytic review of experiments examining the effects of extrinsic rewards on intrinsic motivation. Psychological bulletin, 125(6):627, 1999. URL http://psycnet. apa .org/ journals/bul/125/6/627.

[39] E. L. Deci, R. Koestner, and R. M. Ryan. Extrinsic rewards and intrinsic motivation in education: Reconsidered once again. Review of educational research, 71(1):1-27, 2001. URL http://rer. sagepub. com/content/71/1/1. short.

[40] S. Deterding. Gamification: Designing for motivation. interactions, 19(4):1417, July 2012. ISSN 1072-5520. doi: 10.1145/2212877.2212883. URL http: //dl. acm.org/citation. cfm?doid=2212877. 2212883.

[41] S. Deterding, D. Dixon, R. Khaled, and L. Nacke. From game design elements to gamefulness: Defining "gamification". In Proceedings of the 15th International Academic MindTrek Conference: Envisioning Future Media Environments, MindTrek '11, pages 9-15, New York, NY, USA, 2011. ACM. ISBN 978-1-4503-0816-8. doi: 10.1145/2181037.2181040. URL http://doi. acm. org/10.1145/2181037.2181040.

[42] S. Deterding, M. Sicart, L. Nacke, K. O'Hara, and D. Dixon. Gamification. using game-design elements in non-gaming contexts. In CHI '11 Extended $\mathrm{Ab}$ stracts on Human Factors in Computing Systems, CHI EA '11, pages 2425-2428, New York, NY, USA, 2011. ACM. ISBN 978-1-4503-0268-5. doi: 10.1145/ 1979742.1979575. URL http://doi. acm. org/10.1145/1979742.1979575.

[43] N. du Toit. Designing an interface to provide new functionality for the postprocessing of Web-based annotations. PhD thesis, University of Cape Town, 2014. URL http://pubs.cs .uct.ac.za:1081/archive/00000960/.

[44] J. S. Dumas and J. Redish. A practical guide to usability testing. Intellect Books, 1999. URL https://goo.gl/ccauWm.

[45] I. Frommholz, H. Brocks, U. Thiel, E. Neuhold, L. Iannone, G. Semeraro, M. Berardi, and M. Ceci. Research and Advanced Technology for Digital Libraries: 7th European Conference, ECDL 2003 Trondheim, Norway, August 1722, 2003 Proceedings, chapter Document-Centered Collaboration for Scholars in the Humanities - The COLLATE System, pages 434-445. Springer Berlin Heidelberg, Berlin, Heidelberg, 2003. ISBN 978-3-540-45175-4. doi: 10.1007/978-3-540-45175-4_40. URL http://dx.doi.org/10.1007/978-3$540-45175-4-40$.

[46] X. Fu, T. Ciszek, G. Marchionini, and P. Solomon. Annotating the web: An exploratory study of web users' needs for personal annotation tools. 
Proceedings of the American Society for Information Science and Technology, 42 (1), 2005. ISSN 00447870. doi: 10.1002/meet.14504201151. URL http: //doi.wiley.com/10.1002/meet.14504201151.

[47] N. Fuhr, P. Hansen, M. Mabe, A. Micsik, and I. Sølvberg. Digital libraries: A generic classification and evaluation scheme. In Proceedings of the 5th European Conference on Research and Advanced Technology for Digital Libraries, ECDL '01, pages 187-199, London, UK, UK, 2001. Springer-Verlag. ISBN 3-54042537-3. URL http://dl. acm.org/citation. cfm?id=646634. 699905.

[48] J. Hamari, J. Koivisto, and H. Sarsa. Does gamification work? - a literature review of empirical studies on gamification. In 2014 47th Hawaii International Conference on System Sciences, pages 3025-3034, Jan 2014. doi: 10.1109/ HICSS.2014.377. URL http://dx.doi.org/10.1109/HICSS .2014.377.

[49] C. Hampson, M. Agosti, N. Orio, E. Bailey, S. Lawless, O. Conlan, and V. Wade. The CULTURA project: Supporting next generation interaction with digital cultural heritage collections. In Progress in Cultural Heritage Preservation, volume 7616 of Lecture Notes in Computer Science, pages 668-675. Springer Berlin Heidelberg, 2012. ISBN 978-3-642-34233-2. doi: 10.1007/978-3-642-34234-9_70. URL http://dx.doi.org/10.1007/978-3642-34234-9_70.

[50] C. Hampson, E. Bailey, G. Munnelly, S. Lawless, and O. Conlan. Dynamic personalisation for digital cultural heritage collections. In Proceedings of the 6th International Workshop on Personalized Access to Cultural Heritage, 2013. URL http://ceur-ws . org/Vol-997/patch2013_paper_1.pdf.

[51] B. Haslhofer, W. Jochum, R. King, C. Sadilek, and K. Schellner. The LEMO annotation framework: weaving multimedia annotations with the web. International Journal on Digital Libraries, 10(1):15-32, 2009. ISSN 14325012. doi: 10.1007/s00799-009-0050-8. URL http://dx.doi.org/10.1007/ s00799-009-0050-8.

[52] B. Haslhofer, R. Simon, R. Sanderson, and H. Van de Sompel. The Open Annotation Collaboration (OAC) model. In Multimedia on the Web (MMWeb), 2011 Workshop on, pages 5-9, Sept 2011. doi: 10.1109/MMWeb. 2011.21. URL http://ieeexplore.ieee.org/xpls/abs_all.jsp?arnumber= $6167821 \&$ tag $=1$.

[53] R. S. R. v. d. S. H. Haslhofer, Bernhard Sanderson. Open annotations on multimedia web resources. Multimedia Tools and Applications, 70(2):847-867, 
2014. ISSN 1573-7721. doi: 10.1007/s11042-012-1098-9. URL http://dx. doi .org/10.1007/s11042-012-1098-9.

[54] B. Horowitz. From Rap Genius to Genius - Ben's Blog, July 2014. URL http://www. bhorowitz.com/from_rap_genius_to_genius. [Accessed Online: 2014-10-06].

[55] J. Hunter. Collaborative semantic tagging and annotation systems. Annual Review of Information Science and Technology, 43(1):1-84, 2009. ISSN 1550-8382. doi: 10.1002/aris.2009.1440430111. URL http://dx.doi.org/ 10.1002/aris.2009.1440430111.

[56] J. Kahan, M.-R. Koivunen, E. Prud'Hommeaux, and R. Swick. Annotea: an open RDF infrastructure for shared web annotations. Computer Networks, 39(5):589-608, 2002. ISSN 1389-1286. doi: 10.1016/S13891286(02)00220-7. URL http://www.sciencedirect.com/science/article/ pii/S1389128602002207.

[57] M.-R. Koivunen. Annotea and Semantic Web supported collaboration. In Proceedings of the User Aspects of the Semantic Web (User-SWeb) Workshop, pages 5-16, 2005. URL http://ceur-ws . org/Vol-137/01_koivunen_final. pdf.

[58] M.-R. Koivunen. Annotea Project, October 2005. URL http://www .w3 . org/ 2001/Annotea/. [Accessed Online: 2014-05-06].

[59] M.-R. Koivunen. Semantic authoring by tagging with Annotea social bookmarks and topics. In Proceedings of SAAW2006, 1st Semantic Authoring and Annotation Workshop, Athens, Greece, November 2006. URL http://www. annotea. org/2006/SAAW/annoteaauthoring. pdf.

[60] M.-R. Koivunen and R. R. Swick. Metadata based annotation infrastructure offers flexibility and extensibility for collaborative applications and beyond. In Semannot@ K-CAP 2001, 2001. URL https://www.researchgate.net/profile/Ralph_Swick/publication/ 2380691_Metadata_Based_Annotation_Infrastructure_offers_ Flexibility_and_Extensibility_for_Collaborative_Applications_ and_Beyond/links/53df85910cf2aede4b4908a7.pdf.

[61] M.-R. Koivunen, R. Swick, and E. Prud'hommeaux. Annotea shared bookmarks. In Proceedings of the Annotation Workshop, KCAP '03, 2003. URL http://ftp. informatik. rwth-aachen. de/Publications/CEURWS/Vol-101/Marja-Riitta_Koivunen-et-al.pdf. 
[62] J. Kumar. Gamification at Work: Designing Engaging Business Software, pages 528-537. Springer Berlin Heidelberg, Berlin, Heidelberg, 2013. ISBN 978-3642-39241-2. doi: 10.1007/978-3-642-39241-2_58. URL http://dx.doi.org/ $10.1007 / 978-3-642-39241-2 \_58$.

[63] S. K. Lam and J. Riedl. The past, present, and future of Wikipedia. Computer, 44(3):87-90, March 2011. ISSN 0018-9162. doi: 10.1109/MC. 2011.94. URL http://ieeexplore. ieee.org/lpdocs/epic03/wrapper.htm? arnumber $=5731572$.

[64] T. Lehman and I. Zechory. Introducing Genius.com I Genius, 2014. URL http://meta.genius.com/Genius-founders-introducinggeniuscom-annotated. [Accessed Online: 2014-10-05].

[65] A. M. Lund. Measuring usability with the USE questionnaire. URL http:// www.stcsig.org/usability/newsletter/0110_measuring_with_use.html. [Accessed Online: 2014-06-24].

[66] J. K. Maina and H. Suleman. Enhancing digital heritage archives using gamified annotations. In B. R. Allen, J. Hunter, and L. M. Zeng, editors, Digital Libraries: Providing Quality Information. Proceedings of the 17th International Conference on Asia-Pacific Digital Libraries (ICADL), pages 169-179. Springer International Publishing, Seoul, Korea, 2015. ISBN 978-3-319-27974-9. doi: 10.1007/978-3-319-27974-9_17. URL http://dx.doi.org/10.1007/978-3319-27974-9_17.

[67] C. C. Marshall. Annotation: From paper books to the digital library. In Proceedings of the Second ACM International Conference on Digital Libraries, DL '97, pages 131-140, New York, NY, USA, 1997. ACM. ISBN 0-89791-868-1. doi: 10.1145/263690.263806. URL http://doi.acm.org/10.1145/263690. 263806.

[68] P. McDonald. Game over? When play becomes mechanical I Planning in High Heels, November 2010. URL https://planninginhighheels.com/ 2010/11/25/game-over-when-play-becomes-mechanical/. [Accessed Online: 2016-10-24].

[69] E. D. Mekler, F. Brühlmann, K. Opwis, and A. N. Tuch. Disassembling gamification: The effects of points and meaning on user motivation and performance. In CHI '13 Extended Abstracts on Human Factors in Computing Systems, CHI EA '13, pages 1137-1142, New York, NY, USA, 2013. ACM. ISBN 978-14503-1952-2. doi: 10.1145/2468356.2468559. URL http: //doi . acm. org/10. $1145 / 2468356.2468559$. 
[70] E. D. Mekler, F. Brühlmann, K. Opwis, and A. N. Tuch. Do points, levels and leaderboards harm intrinsic motivation?: An empirical analysis of common gamification elements. In Proceedings of the First International Conference on Gameful Design, Research, and Applications, Gamification '13, pages 66-73, New York, NY, USA, 2013. ACM. ISBN 978-1-4503-2815-9. doi: 10.1145/ 2583008.2583017. URL http://doi . acm.org/10.1145/2583008.2583017.

[71] C. I. Muntean. Raising engagement in e-learning through gamification. In Proc. 6th International Conference on Virtual Learning ICVL, pages 323-329, 2011. URL http://icvl.eu/2011/disc/icvl/documente/pdf/met/ICVL_ ModelsAndMethodologies_paper42.pdf.

[72] D. M. Nichols, D. Pemberton, S. Dalhoumi, O. Larouk, C. Belisle, and M. B. Twidale. Research and Advanced Technology for Digital Libraries: 4th European Conference, ECDL 2000 Lisbon, Portugal, September 18-20, 2000 Proceedings, chapter DEBORA: Developing an Interface to Support Collaboration in a Digital Library, pages 239-248. Springer Berlin Heidelberg, Berlin, Heidelberg, 2000. ISBN 978-3-540-45268-3. doi: 10.1007/3-540-45268-0_22. URL http://dx.doi.org/10.1007/3-540-45268-0_22.

[73] S. Nicholson. A user-centered theoretical framework for meaningful gamification. Games + Learning + Society, 8(1), 2012. URL http://www .quilageo. com/wp-content/uploads/2013/07/Frameworkfor-Meaningful-Gamifications.pdf.

[74] O. Nov and C. Ye. Why do people tag?: Motivations for photo tagging. Commun. ACM, 53(7):128-131, July 2010. ISSN 0001-0782. doi: 10.1145/ 1785414.1785450. URL http://doi . acm.org/10.1145/1785414.1785450.

[75] J. Oomen and L. Aroyo. Crowdsourcing in the cultural heritage domain: Opportunities and challenges. In Proceedings of the 5th International Conference on Communities and Technologies, C\&\#38;T '11, pages 138-149, New York, NY, USA, 2011. ACM. ISBN 978-1-4503-0824-3. doi: 10.1145/2103354.2103373. URL http://doi.acm.org/10.1145/2103354.2103373.

[76] T. Owens. Digital cultural heritage and the crowd. Curator: The Museum Journal, 56(1):121-130, 2013. ISSN 2151-6952. doi: 10.1111/cura.12012. URL http://dx.doi.org/10.1111/cura.12012.

[77] G. Richter, D. R. Raban, and S. Rafaeli. Studying Gamification: The Effect of Rewards and Incentives on Motivation, pages 21-46. Springer International Publishing, Cham, 2015. ISBN 978-3-319-10208-5. doi: 10.1007/978-3-31910208-5_2. URL http://dx.doi.org/10.1007/978-3-319-10208-5_2. 
[78] M. Robertson. Can't play, won't play I Hide \& Seek, October 2010. URL http://hideandseek.net/2010/10/06/cant-play-wont-play/. [Accessed Online: 2015-06-16].

[79] R. M. Ryan and E. L. Deci. Intrinsic and extrinsic motivations: Classic definitions and new directions. Contemporary Educational Psychology, 25 (1):54 - 67, 2000. ISSN 0361-476X. doi: http://dx.doi.org/10.1006/ceps. 1999.1020. URL http://www.sciencedirect.com/science/article/pii/ S0361476X99910202.

[80] M. Sailer, J. Hense, H. Mandl, and M. Klevers. Psychological perspectives on motivation through gamification. IxDEA, 19:28-37, 2013. URL http: //www.fml.mw.tum.de/fml/images/Publikationen/19_2.pdf.

[81] R. Sanderson and H. Van de Sompel. Making Web annotations persistent over time. In Proceedings of the 10th Annual Joint Conference on Digital Libraries, JCDL '10, pages 1-10, New York, NY, USA, 2010. ACM. ISBN 978-1-45030085-8. doi: 10.1145/1816123.1816125. URL http://dl . acm.org/citation. cfm?id=1816123. 1816125 .

[82] R. Sanderson, P. Ciccarese, and H. Van de Sompel. Designing the W3C Open Annotation Data Model. In Proceedings of the 5th Annual ACM Web Science Conference, WebSci '13, pages 366-375, New York, NY, USA, 2013. ACM. ISBN 978-1-4503-1889-1. doi: 10.1145/2464464.2464474. URL http: //doi.acm.org/10.1145/2464464.2464474.

[83] B. Schandl, B. Haslhofer, T. Bürger, A. Langegger, and W. Halb. Linked data and multimedia: the state of affairs. Multimedia Tools and Applications, 59 (2):523-556, 2012. ISSN 1573-7721. doi: 10.1007/s11042-011-0762-9. URL http://dx.doi.org/10.1007/s11042-011-0762-9.

[84] J. Schlötterer, C. Seifert, L. Wagner, and M. Granitzer. A game with a purpose to access Europe's cultural treasure. In GamifIR@ ECIR, pages 13-18, 2015. URL http://ceur-ws.org/Vol-1345/gamifir15_2.pdf.

[85] R. Schroeter, J. Hunter, and D. Kosovic. Vannotea: A collaborative video indexing, annotation and discussion system for broadband networks. pages 1-8, 2003. URL http://espace.library .uq. edu.au/view/UQ:7897.

[86] R. Schroeter, J. Hunter, J. Guerin, I. Khan, and M. Henderson. A synchronous multimedia annotation system for secure collaboratories. In Second IEEE International Conference on e-Science and Grid Computing, e-Science '06, pages 41-41. IEEE, December 2006. ISBN 0-7695-2734-5. doi: 10.1109/E-SCIENCE. 
2006.261125. URL http://ieeexplore. ieee.org/lpdocs/epic03/wrapper. htm?arnumber $=4031014$.

[87] R. Schroeter, J. Hunter, and A. Newman. Annotating relationships between multiple mixed-media digital objects by extending Annotea. In The Semantic Web: Research and Applications, volume 4519 of Lecture Notes in Computer Science, pages 533-548. Springer Berlin Heidelberg, 2007. ISBN 978-3-54072666-1. doi: 10.1007/978-3-540-72667-8_38. URL http://link. springer. com/chapter/10.1007/978-3-540-72667-8_38.

[88] H. Sharp, Y. Rogers, and J. Preece. Interaction Design: Beyond human-computer interaction. John Wiley \& Sons, 2007. ISBN 9780470018668. URL http://idbook. com/.

[89] N. Stenning. Annotator Technical Overview, June 2013. URL https: //speakerdeck. com/nickstenning/annotator-technical-overview. [Accessed Online: 2014-07-25].

[90] H. Suleman. Digital libraries without databases: The bleek and lloyd collection. In Research and Advanced Technology for Digital Libraries, volume 4675 of Lecture Notes in Computer Science, pages 392-403. Springer Berlin Heidelberg, 2007. ISBN 978-3-540-74850-2. doi: 10.1007/978-3-540-74851-9_33. URL http://dx.doi.org/10.1007/978-3-540-74851-9_33.

[91] M. S. Sweetnam, M. Agosti, N. Orio, C. Ponchia, C. M. Steiner, E. C. Hillemann, M. Ó Siochrú, and S. Lawless. User needs for enhanced engagement with cultural heritage collections. In Theory and Practice of Digital Libraries, volume 7489 of Lecture Notes in Computer Science, pages 64-75. Springer Berlin Heidelberg, 2012. ISBN 978-3-642-33289-0. doi: 10.1007/978-3-64233290-6_8. URL http://dx.doi.org/10.1007/978-3-642-33290-6_8.

[92] U. Thiel, H. Brocks, I. Frommholz, A. Dirsch-Weigand, J. Keiper, A. Stein, and J. E. Neuhold. Collate - a collaboratory supporting research on historic european films. International Journal on Digital Libraries, 4(1):8-12, 2004. ISSN 1432-1300. doi: 10.1007/s00799-003-0069-1. URL http://dx.doi.org/10. 1007/s00799-003-0069-1.

[93] H. Van de Sompel, M. L. Nelson, R. Sanderson, L. Balakireva, S. Ainsworth, and H. Shankar. Memento: Time travel for the web. volume abs/0911.1112. 2009. URL http://arxiv. org/abs/0911.1112.

[94] L. von Ahn and L. Dabbish. Designing games with a purpose. Commun. ACM, 51(8):58-67, Aug. 2008. ISSN 0001-0782. doi: 10.1145/1378704. 1378719. URL http://doi . acm.org/10.1145/1378704.1378719. 
[95] M. Wu. The Gamification Backlash + Two Long Term Business Strategies Lithium Community, July 2012. URL https ://community . lithium. com/t5/ Science-of-Social-blog/The-Gamification-Backlash-Two-Long-TermBusiness-Strategies/ba-p/30891. [Accessed Online: 2016-10-24].

[96] G. Zichermann and C. Cunningham. Gamification by design: Implementing game mechanics in web and mobile apps. " O'Reilly Media, Inc.", 2011. URL http://shop. oreilly.com/product/0636920014614.do.

[97] A. Ziesemer, L. Müller, and M. Silveira. Gamification aware: Users perception about game elements on non-game context. In Proceedings of the 12th Brazilian Symposium on Human Factors in Computing Systems, IHC '13, pages 276-279, Porto Alegre, Brazil, Brazil, 2013. Brazilian Computer Society. ISBN 978-85-7669-278-2. URL http://dl.acm.org/citation.cfm?id= 2577101.2577164. 


\section{Appendix A}

\section{Annotator Store Rails Engine}

That Annotator Store Engine ${ }^{1}$ is a pluggable library to implement a backend store for Annotator ${ }^{2}$. The engine is packaged as a Ruby gem and published on rubygems.org for use in any supported Ruby on Rails ${ }^{3}$ application.

When included and configured for use in a Rails application, it provides endpoints that provide search and CRUD actions that are useful for the publishing and management of annotations against a database store. See below:

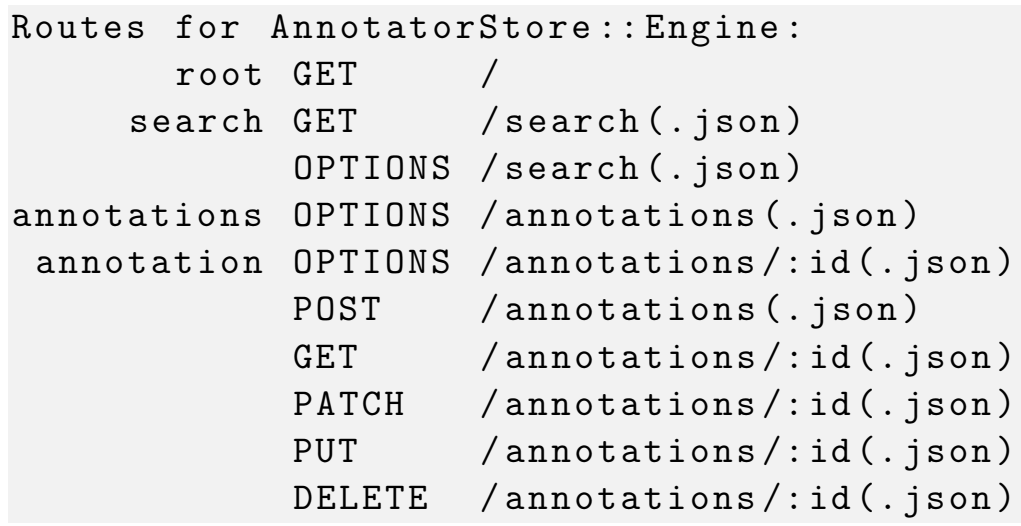

Listing A.1: Endpoints created by the Annnotator Store Rails Engine in a Rails application

\footnotetext{
${ }^{1}$ https://github.com/itsmrwave/annotator_store-gem

${ }^{2}$ http://annotatorjs.org/

${ }^{3}$ http: //rubyonrails.org/
} 



\section{Appendix B}

\section{User Feedback on the Enhanced Digital Heritage Archive}

\section{B.1 Positive Aspects}

Some of the notable user feedback on the positive aspects of the system are itemised below:

- Gamified Users

- "Bravo on creativity!"

- "It's a nice idea to be able to annotate. It was also useful to be able to see other comments and points of view from other users."

- "The images were very clear!"

- "I like the idea of having an archive that makes history available at my fingertips. Very cool! The pictures are interesting and I appreciate all the work that must have been put in to get this archive up."

- "I like that we were able to view and zoom in on the pictures. I liked the organisation of the items in the archive as it made it easy to find the items that I was looking for. There was a lot of information regarding the items which I found informative. The look of the whole website was also very user friendly. Viewing of the notebooks was also very good. As a user, you are able to navigate to the next and previous pages. The colour scheme used also is appropriate for the theme of archive as the theme colours are similar to those used in the watercolours and the pages of the notebook. "

- "Once grasped, annotating seemed easy." 
- "I was able to learn a lot about the Khoisan that I never knew about."

- "I liked the fact that I could comment on any piece at any place on the text or images. It helps that your views can be also be as broad as you want. The art was also amazing as it was relevant in terms of abstract historic art. Truly, all 'art is conceptual' and it is interesting to hear other people's thoughts and being able to place your own. It is like the comment section on Instagram or Facebook."

- "The sign in and log in instructions were clear and easy to follow."

\section{- Un-gamified Users}

- "I liked being able to see other users' opinions especially in the text annotations."

- "The features are brilliant and the information shared is amazing. Some of the things I was clueless about were made clearer because the information was well explained.The colour of the images grabbed my attention."

- "It is easily accessible and I was able to draw a lot of information just by browsing."

- "I enjoyed reading the contributors biographies and I liked that their pictures were included too."

- "It was interesting as I have never done something like this before. It was somewhat easy to understand and I did not struggle much with the content even though it did not appeal to me personally."

- "Convenient and easy to navigate!"

- "I liked the ease of access and the tools made available for annotation."

- "The features were unique and it has enough information about heritage. The art helps one develop more questions. The graphics also suit the content."

- "It was easy to use and user friendly too. I was able to read everything quite clearly."

- "The archives are able to translate the story of the images well."

\section{B.2 Negative Aspects}

Some of the notable user feedback on the negative aspects of the system are itemised below: 


\section{- Gamified Users}

- "There was too much text/content!"

- "Unstructured! Comments are on a range of topics. The site wasn't very easy to navigate."

- "It was difficult to navigate at first. The website didn't look appealing."

- "I wasn't always sure what to annotate. It wasn't very clear. I think I may have annotated incorrectly as I wrote similar things to other users."

- "The font and font size may be a problem. I also found it quite hard to add my own input. The use of white colour text in some places may be a problem as that makes it difficult to read. I also don't like the search because it causes a new window to open which makes using the archive complicated and could confuse the user."

- "There were no clear instructions."

- "I didn't like the small font used for the text. Seeing annotations of others and a scoreboard all the time was also distracting."

- "I didn't like the fact that you could not easily move onto the next image which forces you to go back to be able to go to the next one. It would be very useful if the system could show you where you left off from the last time that you logged on. The images were just too many, I would have loved to view them all, but they were just too many!"

- "At first I wasn't too sure what I was supposed to do, so I wish the instructions could be made a little more clearer."

- "The web page needs some work in terms of Web design. I understand it is more concerned with content but if the loose ends were worked on a little more it would be smoother to browse."

- "It should be made more clear where to go on the home page. Maybe put the tab at the top?"

\section{- Un-gamified Users}

- "The home button and heading are too close to the text. The yellow colour background of the text is not viewable perfectly on a smartphone.'

- "Reading the text in the archive was a difficulty for me. I strongly suggest that you somehow present your information in short and change the font. My complaint is based on how you present your words. Keep the information short but meaningful." 
- "It can get a bit too overwhelming browsing through everything."

- "The formatting and design was really not user-friendly. The related information was all separate and not linked together via hyperlinks or text. The metadata records for the images were not adequate in some cases. I also noticed that there is no contact information."

- "It all seems kind of dark ages. There's this old fashioned feel to the entire website which may seem very vague, but I have no better way of describing it. It could possibly seem that way because it is about the work from ages ago. Maybe the rustic old fashioned feel was intentional."

- "I found some of the words a bit difficult to comprehend."

- "There was lack of background information and outside links that would give me further insight."

- "You should find a better strategy for developing comments and how to display the comments. The font is not well chosen. I believe that if you can display the information properly it will generate more interest."

- "Complicated. Busy. I don't understand what's going on here. It takes too long to figure out how to do things." 